\title{
Non-equilibrium Statistical Mechanics of a Two-temperature Ising Ring With Conserved Dynamics
}

\author{
Nicholas J. Borchers \\ Dissertation submitted to the Faculty of the \\ Virginia Polytechnic Institute and State University \\ in partial fulfillment of the requirements for the degree of
}

Doctor of Philosophy

in

Physics

Michel Pleimling, Chair

Uwe Tauber

Shengfeng Cheng

William Mather

April 30, 2015

Blacksburg, Virginia

Keywords: Non-equilibrium Physics, Phase Transitions, Critical Phenomena Copyright 2015, Nicholas J. Borchers 


\title{
Non-equilibrium Statistical Mechanics of a Two-temperature Ising Ring With Conserved Dynamics
}

\author{
Nicholas J. Borchers
}

(ABSTRACT)

The statistical mechanics of a one-dimensional Ising model in thermal equilibrium is wellestablished, textbook material. Yet, when driven far from equilibrium by coupling two sectors to two baths at different temperatures, it exhibits remarkable phenomena, including an unexpected 'freezing by heating.' These phenomena are explored through systematic numerical simulations. This study reveals complicated relaxation processes as well as a crossover between two very different steady state regimes which are found to be bistable within a certain parameter range.

This work is supported in part by the US National Science Foundation through Grants No. DMR-0904999, DMR-1205309, and DMR-1244666. 


\section{Dedication}

For my Mom. A Ph.D. has never meant more to someone than this has to her. From getting me my first microscope as a kid, to keeping me on track through difficult years when I lost my way, she's supported me every step of the way. I couldn't have done this without her. 


\section{Acknowledgments}

I would like to express my gratitude to my advisor Michel Pleimling and my unofficial advisor Royce Zia. Both Michel and Royce have spent many hours with me trying to unravel the mysterious of non-equlibrium systems, and have shown the patience to entertain my desire to search for fundamental results within this field.

I'd also like to thank Uwe Tauber for both his thorough and critical reading of this document, as well as his excellent courses over the years which have greatly deepened my understanding of physics in general.

Finally, I would like to give my deepest thanks to my wife Hayley. Hayley dear, the only thing that has eclipsed this accomplishment in my life has been meeting you, and building our life together. Funny how these two things have coincided so closely! 


\section{Contents}

List of Figures

List of Tables $\quad$ xiii

1 Introduction $\quad 1$

1.1 Equilibrium statistical mechanics overview . . . . . . . . . . . . 2

1.2 Non-equilibrium approaches . . . . . . . . . . . . . . . . . . . 4

1.3 The Ising model . . . . . . . . . . . . . . . . . . . . 7

1.4 Non-equilibrium Ising models . . . . . . . . . . . . . . . . . . 11

1.5 Organization of this study . . . . . . . . . . . . . . . 13

2 Model Description $\quad 14$

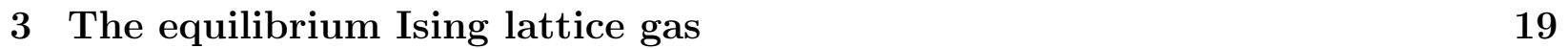

3.1 Standard homogeneous Ising model . . . . . . . . . . . . . . . . . . 19

3.2 The 2J model . . . . . . . . . . . . . . . . . . . . . 26

3.3 Small system analysis . . . . . . . . . . . . . . . . . . 27

4 The two-temperature non-equilibrium model $\quad 34$

4.1 Steady state space-time plots . . . . . . . . . . . . . . . . 34

4.2 Relaxation process . . . . . . . . . . . . . . . 36

4.3 Steady state properties . . . . . . . . . . . . . . . . . 45

4.4 Small system analysis . . . . . . . . . . . . . . . . . . . . 52 
5 Conclusion

$\begin{array}{ll}\text { Bibliography } & 62\end{array}$

$\begin{array}{ll}\text { A Equilibrium Ising model } & 67\end{array}$

$\begin{array}{ll}\text { B Some remarks on a possible phase transition } & 70\end{array}$ 


\section{List of Figures}

1.1 A simple diagram of the Ising model in two dimensions. Spins are fixed to a lattice and may point either up or down. . . . . . . . . . . . .

2.1 Sketch of the two-temperature Ising ring model studied in this paper. Whereas exchanges across blue borders are accepted with the ordinary Metropolis rate for a system at temperature $T$, exchanges across red borders are accepted with the corresponding rate for a system at temperature $T_{w}$. Reprinted figure with permission from N.Borchers, M. Pleimling, and R.K.P. Zia, Physical Review E, 90(6):062113, 2014. Copyright 2014 by the American Physical Society. . .

2.2 Two cycles of configurations illustrating irreversibility and detailed balance violation in the $2 T$ model $(\mathrm{a}, \mathrm{b})$. By comparison, these cycles are reversible in the $2 J$ model $(\mathrm{c}, \mathrm{d})$. See text for explanation of symbols. Reprinted figure with permission from N.Borchers, M. Pleimling, and R.K.P. Zia, Physical Review E, 90(6):062113, 2014. Copyright 2014 by the American Physical Society. . .

3.1 Log-binning relaxation of the energy density $\langle\mathcal{H}\rangle / L$ for the standard Ising system at temperature $T=1$ and two different lattice sizes $L$. In this plot (as well as in the other figures below showing log-binning relaxation) the average of the quantity of interest is sampled at intervals between $2^{x}$ and $2^{x+1}$ MCS. The equilibrium value of $\langle\mathcal{H}\rangle / L$ is already very close to the value $-\tanh (1) \approx$ -0.76 of the infinite lattice. The data results from averaging over an ensemble of 40 to 100 independent realizations. Error bars are comparable to the sizes of the symbols. Reprinted figure with permission from N.Borchers, M. Pleimling, and R.K.P. Zia, Physical Review E, 90(6):062113, 2014. Copyright 2014 by the American Physical Society. . . . . . . . . . . . . . . . . . . 
3.2 Log-binning relaxation of the energy density for the standard Ising model with $L=512$. The data results from averaging over an ensemble of 40 to 100 independent realizations. Error bars are comparable to the sizes of the symbols. Reprinted figure with permission from N.Borchers, M. Pleimling, and R.K.P. Zia, Physical Review E, 90(6):062113, 2014. Copyright 2014 by the American Physical Society. . . . . . . . . . . . . . . . .

3.3 Convergence of the energy density log-binning for the standard Ising model with $L=256$. The data results from averaging over an ensemble of 50 independent realizations. Filled symbols are for random initial conditions, while empty symbols are for ordered initial conditions. Error bars are comparable to the sizes of the symbols. . . . . . . . . . . . . . . . . .

3.4 Space-time plot of the standard Ising system after it has reached equilibrium at $T=1$. The plot shows the evolution of the system over a total of $2^{12} \mathrm{MCS}$ after an initial relaxation period of $2^{15} \mathrm{MCS}$, where two consecutive horizontal lines are separated by 6 MCS. The system size is $L=512$. . . . . . . . . . .

3.5 Logarithmic space-time plot of the standard Ising system after it has reached equilibrium at $T=1$. The plot shows the evolution of the system over a total of $2^{25}$ MCS after an initial relaxation period of $2^{15}$ MCS. Here, the number of MCS between adjacent horizontal lines increases exponentially as the time proceeds downwards. Initially, each line corresponds to a single MCS, but by the end there are $2^{20}$ MCS between adjacent lines. The system size is $L=512$. 24

3.6 $G(r)$ for the standard equilibrium Ising ring with Kawasaki dynamics for a system of length $L=127$. L is chosen to be odd to ensure a symmetric result about a given reference point. The lines refer to the approximation Eq. (3.3). Reprinted figure with permission from N.Borchers, M. Pleimling, and R.K.P. Zia, Physical Review E, 90(6):062113, 2014. Copyright 2014 by the American Physical Society. . . . . . . . . . . . . . . . . . .

3.7 Equilibrium distributions of the normalized window magnetization $m$ for the standard Ising model with $L=128$ and $w=32$. Reprinted figure with permission from N.Borchers, M. Pleimling, and R.K.P. Zia, Physical Review E, 90(6):062113, 2014. Copyright 2014 by the American Physical Society. . .

3.8 Space-time plots of the $2 J$ model after it has reached equilibrium at $T=1$. The left plot shows the evolution of the system over a total of $2^{12}$ MCS after an initial relaxation period of $2^{15} \mathrm{MCS}$, where two consecutive horizontal lines are separated by 6 MCS. The system size is $L=512$ and $w=128$. The right plot is the logarithmic version of this plot over $2^{25}$ MCS. The boundaries between the regions are highlighted in red. . . . . . . . . . . . . 
3.9 Equilibrium distributions of the normalized window magnetization $m$ for the $2 J$ Ising model with $L=128$ and $w=32$. Distributions for different temperatures are shown. Reprinted figure with permission from N.Borchers, M. Pleimling, and R.K.P. Zia, Physical Review E, 90(6):062113, 2014. Copyright 2014 by the American Physical Society. . . . . . . . . . . . . . . . . . .

3.10 The left image displays the configuration network for Kawasaki dynamics acting on $L=6, \mathcal{M}=0$. The numbers correspond to the states indicated in Table 3.1, while the colors refer to the corresponding energy level. In the right plot, the calculated values of $P^{*}$ for this system at $T=5$ are indicated.

3.11 The left image displays the configuration network for Kawasaki dynamics acting on the $2 J$ system with $L=6, \mathcal{M}=0$, and $w=2$. The numbers correspond to the states indicated in table 3.2, while the colors refer to the corresponding energy level. In the right plot the calculated values of $P^{*}$ for this system at $T=3$ are indicated. . . . . . . . . . . . . .

4.1 Space-time plots of the two-temperature model at different values of $T$. The plots are from a system of length $L=512$, with a window of size $w=128$ wherein the system is in contact with a reservoir at temperature $T_{w}=\infty$. For the left system we have $T=1$, whereas for the right system $T=1.75$. The boundaries between the regions are highlighted in red. Both plots show the evolution of the system over a total of $2^{12}$ MCS after an initial relaxation period of $2^{30}$ MCS, where two consecutive vertical lines are separated by 6 MCS. . . . . . . . . . . . . . . . . . . . . .

4.2 Space-time plots of the two-temperature model at different values of $w$ and over different lengths of time. The left plot is a $2 T$ system with $L=512$, $w=192$, and $T=1$. For a window of this size, the ordered phase does not form even for this relatively low value of $T$. This plot shows the evolution of the system over a total of $2^{12}$ MCS after an initial relaxation period of $2^{30}$ MCS, where two consecutive vertical lines are separated by 6 MCS. The right plot shows a logarithmic space time for the system with $w=128$. Here the relaxation period is again $2^{30} \mathrm{MCS}$, but a total of $2^{25} \mathrm{MCS}$ of evolution are shown in the plot. The boundaries between the regions are highlighted in red. 
4.3 (a) Log-binning relaxation of the average energy density for $T=1$ with fixed system size $L=512$ and varying window size $w$ (yielding different values for the ratio $R=w / L$, see the figure legend). The dashed vertical line is placed at the point at which the green curve with $w=128$ exits the metastable state, which is equivalent to $(w / 2)^{3}=64^{3}=2^{18}$. (b) Log-binning relaxation of the average energy density for fixed ratio $R=w / L=1 / 4$ and $T=1$. The dashed vertical lines are placed to correspond with $(w / 2)^{3}$ for their respective curves (left most curve located at $16^{3}$, right most curve located at $128^{3}$ ). Reprinted figure with permission from N.Borchers, M. Pleimling, and R.K.P. Zia, Physical Review E, 90(6):062113, 2014. Copyright 2014 by the American Physical Society. . . . . . . . . . . . . . . . . . .

4.4 Upper panel: relaxation of the normalized average window magnetization for $R=1 / 4$ and $T=1$, see main text. Here the dashed vertical lines correspond with $(2 w)^{3}$. Lower panel: the same for the standard Ising model. Reprinted figure with permission from N.Borchers, M. Pleimling, and R.K.P. Zia, Physical Review E, 90(6):062113, 2014. Copyright 2014 by the American Physical Society. . . . . . . . . . . . . . . . . . . .

4.5 Log-binning relaxation of the average energy density for $L=128, T=1$, and various values of the ratio $R=w / L$. Error bars are comparable to the symbol size. Reprinted figure with permission from N.Borchers, M. Pleimling, and R.K.P. Zia, Physical Review E, 90(6):062113, 2014. Copyright 2014 by the American Physical Society. . . . . . . . . . . . . . . . . . . .

4.6 Convergence of the energy density log-binning for the $2 T$ Ising model with $L=256$ and $w=64$. The data results from averaging over an ensemble of 50 independent realizations. Filled symbols are for random initial conditions, while open symbols are for ordered initial conditions. Error bars are comparable to the sizes of the symbols. . . . . . . . . . . . . .

4.7 Log binning of the energy density for the standard equilibrium model with $L=1024$ and $T=1$. The different curves result from using different initial conditions for the ensemble of 50 independent realizations. . . . . . . . . . .

4.8 Log binning of the energy density for the $2 T$ with $L=1024, w=256$, and $T=1$. The different curves result from using different initial conditions for the ensemble of 50 independent realizations. . . . . . . . . . . . . .

4.9 A sample of six simulations from the fifty used to construct the steady state curve in 4.8. . . . . . . . . . . . . . . . . . . . . . 
$4.10 G((w+1) / 2, r)$ for the two-temperature Ising $\operatorname{ring}$ with $L=127, w=31$, and various values of $T$. Here the lattice site $i=(w+1) / 2$ is the site in the middle of the window of width $w$. The end of the window is indicated by the dashed vertical line. Reprinted figure with permission from N.Borchers, M. Pleimling, and R.K.P. Zia, Physical Review E, 90(6):062113, 2014. Copyright 2014 by the American Physical Society. . . . . . . . . . . . . . . . . . .

4.11 Average bond energy $u_{i}$ at site $i$ for $L=128, T=1$, and various ratios $R=w / L$. The solid magenta horizontal line indicates the equilibrium energy of the standard model at $T=1$. The dashed vertical lines are placed to demonstrate the end of the window region for the $R=1 / 4$ curve. For all curves the borders of the infinite temperature window are marked by minima in $u_{i}$ followed by discontinuous transitions to maxima outside the window. Reprinted figure with permission from N.Borchers, M. Pleimling, and R.K.P. Zia, Physical Review E, 90(6):062113, 2014. Copyright 2014 by the American Physical Society. . . . . . . . . . . . . . . . . .

4.12 Distributions for the normalized window magnetization $m$ with system parameters $L=128$ and $R=1 / 4(w=32)$. Results for different values of $T$ are shown. Reprinted figure with permission from N.Borchers, M. Pleimling, and R.K.P. Zia, Physical Review E, 90(6):062113, 2014. Copyright 2014 by the American Physical Society. . . . . . . . . . . . . . . . .

4.13 Distributions for the normalized window magnetization $m$ with $L=128$, $T=1$, and various window sizes. Reprinted figure with permission from N.Borchers, M. Pleimling, and R.K.P. Zia, Physical Review E, 90(6):062113, 2014. Copyright 2014 by the American Physical Society. . . . . . . . . . . .

4.14 Distributions for the normalized window magnetization $m$ with $R=1 / 4$, $T=1$, and various system sizes. The curves are generated by averaging over 200 independent realizations, each with $2^{30}$ MCS of relaxation and $2^{20}$ simulation MCS. . . . . . . . . . . . . . . . . . .

4.15 Distributions for the average system energy density $\langle\mathcal{H}\rangle / L$ with $R=1 / 4$, $T=1$, and various system sizes. The curves are generated by averaging over 200 independent realizations, each with $2^{30}$ MCS of relaxation and $2^{20}$ simulation MCS. . . . . . . . . . . . . . . . . . . .

4.16 Left: Distributions for the normalized window magnetization $m$ with $L=$ 1024, $T=1$, and two values of $R$. Right: The same as the left, but for the average energy density $\langle\mathcal{H}\rangle / L$ instead. Both curves are generated by averaging over 200 independent realizations, each with $2^{30}$ MCS of relaxation and $2^{20}$ simulation MCS. 
4.17 The left image displays the configuration network for Kawasaki dynamics acting on $L=6, \mathcal{M}=0$, and $w=2$. The numbers correspond to the states indicated in Table 3.1, while the colors refer to the corresponding energy level. The directed edges between the states indicate the direction of the probability current, while the color corresponds to the source of energy gain or loss. Red edges indicate energy gained from the infinite temperature reservoir, while blue indicate energy deposited into the low temperature reservoir. Dashed lines indicate zero current. In the right plot, the calculated values of $P^{*}$ for this system at $T=4$ are shown. . . . . . . . . . . . . . . .

4.18 Here the configuration network for Kawasaki dynamics acting on $L=6$, $\mathcal{M}=0$, and $w=2$ is shown again to emphasize the underlying parity symmetry which exists within the model. The left image shows configuration space with the bond representation of the state mapped to each node (excepting the central state of maximum energy which is it's own mirror image) to emphasize the parity symmetry in the model. The central image shows how the symmetric states can be folded together, while the rightmost image displays the reduced network representation. . . . . . . . . . . . . .

4.19 The left image displays the parity reduced configuration network for Kawasaki dynamics acting on $L=6, \mathcal{M}=0$, and $w=2$. The colors still refer to the energy levels indicated in Table 3.1. The directed edges between the states indicate the direction of the probability current, while the color corresponds to the source of energy gain of loss. Red edges indicate energy gained from the infinite temperature reservoir, while blue edges indicate energy deposited into the low temperature reservoir. Dashed lines indicate zero current. In the right plot, the calculated values of $P^{*}$ for this system at $T=4$ are shown. .

4.20 System entropy $S_{\text {sys }}$ versus temperature scaled as $\exp (-4 / T)$ for the $L=6$ model at a variety of window sizes. Note the system entropy for $w=1$ and $w=2$ is lower than the equilibrium value for low values of $T \ldots \ldots$

B.1 The ideal behavior of the order parameter for a continuous phase transition as temperature is varied. . . . . . . . . . . . . . . . . . 71

B.2 The response of the $2 T$ order parameter $|m|$ as $T$ is varied near the crossover temperature. Several system sizes are considered. . . . . . . . . . . .

B.3 The Binder cumulant for the $2 T$ system as a function of $T$. Several system sizes are considered. . . . . . . . . . . . . . . . . . . 


\section{List of Tables}

3.1 The 10 energetic configurations for $L=6$ and $\mathcal{M}=0$. The cell colors correspond to the node colors in Fig. 3.10. . . . . . . . . . . . .

3.2 The 10 energetic configurations for $L=6$ and $\mathcal{M}=0$ in a $2 J$ model with $w=2$. The cell colors correspond to the node colors in Fig. 3.11. . . . . . .

4.1 The 10 energetic configurations of the 2 T model for $L=6, w=2$, and $\mathcal{M}=0$. The bonds which fall within the window are contained within the red vertical lines of the configurations. The cell colors correspond to the node colors in

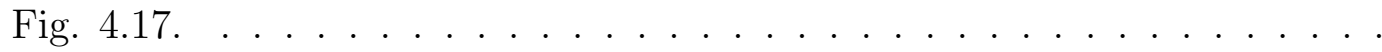




\section{Chapter 1}

\section{Introduction}

This is an expanded version of the introduction found in [1], with numerous additions to provide further background. Reprinted excerpts with permission from N.Borchers, M. Pleimling, and R.K.P. Zia, Physical Review E, 90(6):062113, 2014. Copyright 2014 by the American Physical Society.

The vast majority of interesting phenomena observed in nature arise from many interacting degrees of freedom. Despite this, much of physics, both classical and modern, is an attempt to limit the available degrees of freedom so as to better understand the behavior of the individual components of the system in question. This approach works phenomenally well for, say, describing the motion of a pair of colliding billiard balls or the trajectory of an electron through a bubble chamber. When the phenomenon in question inherently involves many degrees of freedom, however, this program is of limited use regardless of how well determined the space-time evolution of the individual degrees of freedom are. The potential limitations are numerous, but a simple issue which is often encountered is that the sheer number of degrees of freedom prevent a practical simultaneous solution. A prime physical example of this limitation is, of course, the kinetic theory of gases. Still a nascent and unverified theory in the mid-nineteenth century, it would take an essentially new approach to prove this perspective and connect it to the well-established results of thermodynamics for which it was meant to provide the basis. With the advent of statistical mechanics in the late nineteenth century, Boltzmann, Gibbs, and many others provided the framework to accomplish just this. There are a plethora of modern texts which detail the foundations of this theory (see for example $[2,3]$ ), but a few of the more fundamental results will be highlighted here to provide context. 


\subsection{Equilibrium statistical mechanics overview}

By taking a probabilistic approach to dealing with the near innumerable degrees of freedom present in even a modest amount of matter, the macroscopic laws of thermodynamics can be connected to the microscopic degrees of freedom of the constituent molecules. In such an approach, each unique configuration of possible values for the microscopic degrees of freedom can be considered as a single microstate $\mu$ of the system. The collection of all possible microstates of the system forms the configuration space of the system. The number of these microstates which are consistent with the macroscopic physical parameters of the system, such as the internal energy $E$, pressure $P$, or volume $V$, are given by the multiplicity $\Omega(E, P, V)$. As the microscopic degrees of freedom evolve in time, usually via some Hamiltonian $\mathcal{H}(\mu)$, the system follows a path through this space. Rather than following the trajectory of a single system through this configuration space, one of the central tenets of statistical mechanics is to consider an ensemble of similarly prepared systems, and through a statistical understanding of this ensemble to construct the probability distribution $P_{\mu}$ over the configuration space. As a function which represents the probability of a given state $\mu$, $P_{\mu}$ adheres to the following standard constraints for such a function, namely:

$$
\begin{aligned}
& 0 \leq P_{\mu} \leq 1 \\
& \sum_{\mu} P_{\mu}=1
\end{aligned}
$$

This distribution then allows the expectation values of macroscopically observable quantities, $\mathcal{O}(\mu)$, to be computed via the standard averaging procedure:

$$
\langle\mathcal{O}\rangle=\sum_{\mu} \mathcal{O}(\mu) P_{\mu}
$$

This is specific to a system with a finite and discrete configuration space, but it can be generalized in principle to continuous systems by replacing the sum with an integral.

The trick, then, is in determining $P_{\mu}$. In general, this is nearly as daunting a task as tracking the time evolution of the individual degrees of freedom. But great progress was made by considering systems restricted to a particular collection of $\mu$ in some way. For instance, by considering a system which is both mechanically and thermally isolated, the configuration space is limited to only states which satisfy $\mathcal{H}(\mu)=E$, where $E$ is the conserved internal energy of the isolated system in question. This set of microstates is referred to as the microcanonical ensemble, and has a corresponding multiplicity of $\Omega(E)$ (where $P$ and $V$ are held fixed). One of the core postulates of statistical mechanics is that all states of the microcanonical ensemble are equally probable. This immediately fixes the distribution over these states to 


$$
P_{\mu}=\frac{1}{\Omega(E)} .
$$

This assumption may at first appear somewhat arbitrary, but Liouville's theorem confirms this supposition for all systems governed by a Hamiltonian. In any event, the use of this postulate produces reasonable results when applied to systems such as the ideal gas, and provides a path forward for further development.

By utilizing the above result in the context of isolated subsystems allowed to interact only with each other through the exchange of heat (or energy), it is possible to define one of the fundamental results of thermodynamics, namely that the temperature $T$ is related to the change in entropy $S$ in the following manner:

$$
\frac{\partial S}{\partial E}=\frac{1}{T} .
$$

One of the keys to finding this result is a reinterpretation of the equilibrium condition in terms of the microstates of the system. Consider that the total energy is divided amongst the subsystems as $E_{1}$ and $E_{2}$, but that the total energy is conserved such that $E=E_{1}+E_{2}$. If it is argued that in equilibrium the interacting subsystems divide the total energy $E$ in such a way as to maximize the joint multiplicity

$$
\Omega(E)=\Omega_{1}\left(E_{1}\right) \Omega_{2}\left(E-E_{1}\right),
$$

then eqn. 1.4 naturally follows, provided the entropy is defined in terms of the multiplicity as

$$
S(E) \equiv k_{B} \ln \Omega(E) .
$$

This is the famous relation of Boltzmann, and here $k_{B}$ is a proportionality constant named in his honor. It is quite possibly the most famous result of all statistical physics. From this relationship, the equilibrium condition of maximizing $\Omega(E)$ is shown to be identical to maximizing the entropy.

Further results are possible by considering slight variations of the above reasoning. If the adiabatic isolation requirement of the microcanonical ensemble is relaxed and heat is allowed to be exchanged with an environment at constant temperature the number of accessible microstates is increased. This collection of states forms the canonical ensemble, and progress can be made if the equilibrium condition is still enforced such that the system in question comes to have the same temperature as the environment, often called the thermal reservoir or heat bath. As before, the equilibrium condition is tantamount to maximizing the multiplicity with respect to energy exchanges, but here it is the joint multiplicity of the system plus 
environment. Focusing specifically on the distribution over the microstates of the system, and recognizing that, by definition, the energy of the system is small compared to that of the reservoir, the following normalized distribution is found:

$$
P_{\mu}=\frac{e^{-\mathcal{H}(\mu) / k_{B} T}}{Z(T)} .
$$

The normalization constant

$$
Z(T)=\sum_{\mu} e^{-\mathcal{H}(\mu) / k_{B} T}
$$

is known as the partition function. The distribution is called the Boltzmann distribution, and it determines the probability of a given microstate in the canonical distribution. It should be noted that this distribution depends only on the energy of the microstate and the temperature of the reservoir. In this way, it respects the postulate that all configurations of equal energy should be equally probable. The partition function, though only a normalization constant, is an extremely important quantity in its own right, and it serves a central role in the calculation of expectation values of observables. This owes to the fact that, with the distribution set by by eqn. 1.7, $Z$ contains the entirety of the available information on the probability of all microstates since it is a sum of the so-called Boltzmann factors, $e^{-\mathcal{H}(\mu) / k_{B} T}$, over the entire configuration space. It can therefore be used directly to compute averages without recourse to the underlying distribution.

\section{$1.2 \quad$ Non-equilibrium approaches}

While the techniques outlined in the previous section provide a sound basis for understanding physical systems in thermal equilibrium, many of the most fascinating phenomena around us are due to non-equilibrium stochastic processes coupled to more than one reservoir (of energy, particles, etc.). Examples include all living organisms and the life-sustaining oceanatmosphere complex. Despite the critical importance of these phenomena, an overarching principle for far-from-equilibrium statistical mechanics remains elusive. The probabilistic treatment of the numerous degrees of freedom of the these systems still represents the best path forward, but the removal of the equilibrium condition does not allow for a generic solution of the probability distribution $P_{\mu}$ as was done in eqn. 1.7. For systems which remain near equilibrium, linear response theory allows features of these systems to be expressed in terms of properties of the equilibrium system. But for systems which are held far from equilibrium, say in some non-equilibrium steady state (NESS) generated by a constant external drive, no universal properties of $P_{\mu}$ are apparent.

Despite this, considerable recent progress on fluctuation theorems and the 'non-equilibrium 
counterpart' of the free energy has been made. Recently, Seifert provided a comprehensive review of these gains over the past couple decades [4]. Here, exact results for the distribution functions of exchanged heat, applied work, and entropy production allow some generic features of NESS to be established in lieu of exact knowledge of $P_{\mu}$. In particular, the recognition that these central concepts of classical thermodynamics can be meaningfully defined on the level of the individual stochastic trajectories of the system allows them to become accessible to both experiment and simulation. This can lead to surprising results which lend insight into NESS. In the case of entropy production, for instance, this leads to the seemingly counter-intuitive result that certain trajectories may produce negative values. While in apparent contradiction to the second law of thermodynamics, this can be resolved if the second law is understood to apply to the average entropy production.

These results can be established for either continuous or discrete systems. In the case of continuous variables, the individual trajectories are described by a Langevin equation and, if the Kramers-Moyal expansion terminates after the second moment, the Fokker-Planck equation is used to describe the evolution of the distribution of an entire ensemble. For systems with a discrete set of states, the analogous approach is to define a set of rates for the dynamics between states and use the master equation to describe the distribution on the ensemble level. Since this study will focus on a discrete model, the latter approach will prove to be more appropriate here. Such a formulation of the underlying physics has the added benefit that it is particularly well-suited to computer simulations. For these reasons, we will proceed by providing more detail on how the dynamical rates and master equation are used in general.

The discussion of the configuration space distribution $P_{\mu}$ has thus far treated this quantity as a constant feature of the physical system in question. Implicit in this treatment was the assumption that the system is in a so called steady state in which $P_{\mu}$ is indeed constant. For equilibrium systems this is a natural assumption, as the state of thermal equilibrium is simply a special type of steady state. Once non-equilibrium systems are considered, it is necessary to account for the manner in which $P_{\mu}$ varies in time. Limiting ourselves to a discrete time step $\tau$ for convenience, the time-dependent distribution can be written as $P_{\mu}(\tau)$. If we assume that the time evolution of the system only depends on the current configuration of the system and some static transition probability, then the system is said to be Markovian and the future value of $P_{\mu}$ only depends on the current value, i.e. $P_{\mu}(\tau+1) \propto P_{\mu}(\tau)$. The time evolution may thus be described by

$$
\begin{aligned}
P_{i}(\tau+1) & =\sum_{j} W_{i}^{j} P_{j}(\tau) \\
& =\sum_{j \neq i} W_{i}^{j} P_{j}(\tau)+W_{i}^{i} P_{i}(\tau),
\end{aligned}
$$

where the $W_{i}^{j}$ are the transition rates from state $j$ to $i[5]$. The rates can, in principle, 
depend on time, but to allow for the simple relationship of eqn. 1.9 this complication will not be considered here. For $P_{\mu}$ to follow the constraints of eqn. 1.1 over time, i.e. for probability to be conserved, the $W_{i}^{j}$ must follow an identical set of constraints. The master equation is constructed by then considering the "instantaneous" change in probability of the $i^{\text {th }}$ state, $P_{i}$,

$$
\begin{aligned}
\Delta P_{i}(\tau) & \equiv P_{i}(\tau+1)-P_{i}(\tau) \\
& =\sum_{j \neq i} W_{i}^{j} P_{j}(\tau)+\left(W_{i}^{i}-1\right) P_{i}(\tau) \\
& =\sum_{j \neq i}\left[W_{i}^{j} P_{j}(\tau)-W_{j}^{i} P_{i}(\tau)\right] .
\end{aligned}
$$

The equation can be written more succinctly as

$$
\Delta P_{i}(\tau)=\sum_{j} L_{i}^{j} P_{j}(\tau)
$$

with

$$
L_{i}^{j}= \begin{cases}W_{i}^{j}, & i \neq j \\ -\sum_{k \neq j} W_{k}^{j}, & i=j .\end{cases}
$$

While the master equation provides a relationship for the time-evolution of the distribution for all Markovian stochastic processes, it is particularly useful when used to examine the steady state distribution $P_{\mu}^{*}$. The defining feature of the steady state is that the average features of the system appear the same regardless of when the system is measured. One way to express this quality is to say that $P_{\mu}^{*}(\tau+1)=P_{\mu}^{*}(\tau)$. This is equivalent to setting the left hand side of eqn. 1.10 to zero. The steady state condition can then be expressed as

$$
\sum_{j \neq i}\left[W_{i}^{j} P_{j}^{*}-W_{j}^{i} P_{i}^{*}\right]=0
$$

The steady state distribution $P_{\mu}^{*}$ which satisfies this expression must necessarily be stationary in time. This steady state condition should not be confused with the equilibrium condition. A system in equilibrium is not only in a steady state in which the average features of the system are independent of time, but further respects time reversal symmetry such that any trajectory in configuration space occurs with the same probability as the reverse process. On the level of an individual time step, this is akin to the relationship 


$$
W_{i}^{j} P_{j}^{*}=W_{j}^{i} P_{i}^{*} .
$$

From this equality, which is known as detailed balance, eqn. 1.13 is trivially zero since each term of the sum vanishes. This guarantees that any $P_{\mu}^{*}$ which satisfies detailed balance has entered an equilibrium steady state, but this is clearly not the only manner by which the sum in eqn. 1.13 can be zero. All systems which satisfy eqn. 1.13 but not the detailed balance condition of eqn. 1.14 are said to reside in a NESS with a (generally) non-trivial $P_{\mu}^{*}$. In particular, even when a system evolves according to the master equation with timeindependent rates, the probability distribution of the stationary state is usually far from the simple Boltzmann distribution of eqn. 1.7 as soon as the dynamics violates detailed balance. Further, although a systematic construction for this distribution exists [6], finding its analytic form explicitly is a highly non-trivial task. Needless to say, when the rates are themselves time-dependent (e.g., the diurnal or seasonal heating and cooling cycles of our atmosphere), the system displays more intriguing, but less comprehensible, behavior.

In addition, there are persistent stationary probability current loops in such states [7], leading to observable consequences in general. This can be seen clearly if we recognize the quantity within the sum of eqn. 1.10 as the net probability current from state $j$ to $i$,

$$
K_{i}^{j}(\tau) \equiv W_{i}^{j} P_{j}(\tau)-W_{j}^{i} P_{i}(\tau) .
$$

Naturally, these currents immediately vanish in equilibrium due to the detailed balance condition of eqn. 1.14. The master equation, eqn. 1.10, may also be rewritten in terms of these currents as

$$
\Delta P_{i}(\tau)=\sum_{j \neq i} K_{i}^{j}(\tau) .
$$

Though the differences are embedded in the definition of $K_{i}^{j}(\tau)$, eqn. 1.16 should be recognized as nothing more than a continuity equation for the probability $P_{\mu}$. Due to the constraints which enforce probability conservation, this relationship should come as no surprise to those familiar with the flow of other conserved quantities such as mass or charge.

\subsection{The Ising model}

Even with the assortment of techniques and formalisms developed in the previous section, the typical physical system is far too complex to allow for the exact analytical calculation of the quantities necessary for a complete description. For instance, in an equilibrium system the first step for solving problems usually involves the determination of the partition function, 
since most quantities of interest can be found by taking derivatives of $Z$ with respect to the appropriate parameter. While it is straightforward to write down the sum of eqn. 1.8 , it may be impractical to find a concise form which allows derivatives to be taken conveniently. The situation is, of course, even worse for non-equilibrium systems where $P_{\mu}^{*}$ is unknown and there is no partition function to utilize. The master equation (eqn. 1.10) can be used but quickly becomes intractable due to the overwhelming number of microstates.

One way to make progress, given the general technical difficulties, is to study simple model systems. While these system are, by definition, somewhat crude approximations of actual physical systems, they are eminently more soluble. And with a judicious choice of which features to prune, key aspects of real-world physical phenomena can still be retained and studied. Another key advantage of these simple model systems is that their behavior can often be easily simulated by computers, where the simplicity of these models may allow us to develop a full understanding while providing some insight into non-equilibrium processes.

The use of this type of modeling is ubiquitous and well accepted in nearly all fields of science today, and each discipline has a number well established examples of this approach being used successfully. In the realm of statistical and condensed matter physics, fields particularly wellknown for utilizing this approach, there is perhaps no better example than the Ising model. Initially thought to not even accurately describe the ferromagnetic materials for which it was proposed, the Ising model is now used to help understand cooperative phenomena across the boundary of numerous disciplines. Owing in large part to the extreme simplicity of the model, its applicability is far reaching in that it exposes that "the essential features of cooperative phenomena (especially at the critical point) depend not on the details of intermolecular forces but on the mechanism of propagation of long-range order" [8]. The Ising model is certainly not the only model in which these universal features become apparent, as a whole host of dynamical critical behaviors sharing similar features have been classified into various universality classes [9]. Still, the Ising model represents one of the first models in which such behaviors were revealed, and it has provided motivation for many of the subsequent investigations.

The model was originally proposed in a paper by Wilhelm Lenz in 1920 [10] as means to explain the ferromagnetic phase transtion, in which a ferromagnetic material develops a spontaneous magnetization with the application of an external magnetic field - provided the temperature of the material is held below some critical threshold. Lenz sought a simple physical explanation for this phenomena based on the collective influence of the spin magnetic moments of the individual atoms of the material. A complete description of the orientations and interactions among the atoms of the material would undoubtedly be complex and unwieldy to work with, so Lenz suggested several simplifications to arrive at a model more amenable to study. The material is considered to be comprised of a large number of identical atoms, where:

- Each atom is affixed to a lattice point and is not free to move. 
- Each atom has an associated spin $\sigma_{i}$ which may take one of two orientations.

- The spins of the atoms may only interact with their nearest neighbors within the lattice.

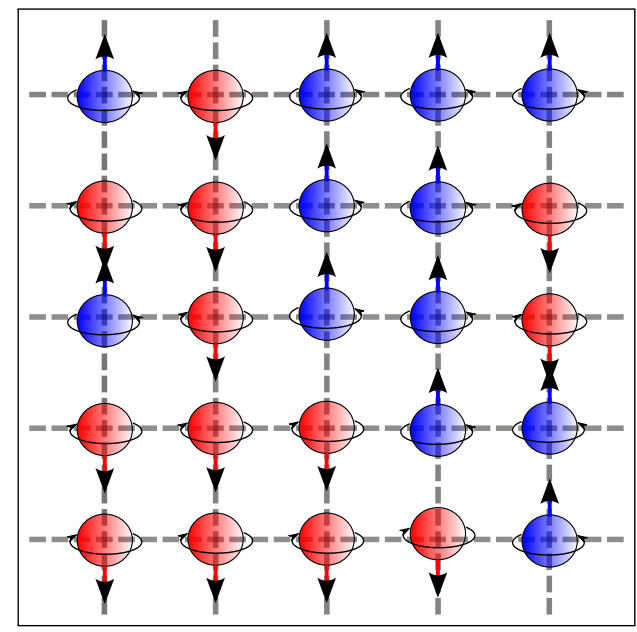

Figure 1.1: A simple diagram of the Ising model in two dimensions. Spins are fixed to a lattice and may point either up or down.

The spins interact with each other and any external magnetic field $h_{j}$ via the Ising Hamiltonian,

$$
\mathcal{H}(\{\sigma\})=-\sum_{\langle i, j\rangle} J_{i j} \sigma_{i} \sigma_{j}-\mu_{S} \sum_{j} h_{j} \sigma_{j},
$$

with the two spin orientations imposed by drawing $\sigma_{i}$ from the set $\{+1,-1\}$. The Hamiltonian defines an energy for an entire configuration of spin orientations, denoted $\{\sigma\}$. The first term of the Hamiltonian reflects the interaction between the spins, the sum being over only adjacent pairs with interaction strength $J_{i j}$ between them. For a ferromagnetic material, the expectation is that neighboring spins tend to point in the same direction, and therefore $J_{i j}$ must be positive to reflect this behavior. The second term of eqn. 1.17 captures how the magnetic moment of the spin, $\mu_{S}$, interacts with the external magnetic field $h_{j}$. In many studies, including this one, the applied field is assumed to be zero, and the behavior which emerges solely from the spin's mutual interactions is explored.

The validity of using such severe approximations to represent an actual ferromagnetic material can certainly be questioned, a fact we will return to briefly, but within the context of this Hamiltonian the problem of exploring the phase transition is now well-posed. For an Ising lattice held in equilibrium at a specific temperature, the partition function, eqn. 1.8, may be constructed with each configuration $\{\sigma\}$ used as the microstate $\mu$. From $Z$, one can derive all thermodynamic potentials and look for indications of the phase transition. 
Ernst Ising, a student of Lenz at Hamburg University, inherited the model in the early 1920s. Ising made several critical contributions to the development of the model, including presenting the Hamiltonian of eqn. 1.17 which dictates the spin interactions. In his doctoral thesis, summarized in [11], Ising derived an exact expression for the partition function for the one dimensional version of the model that now bears his name. Somewhat famously, his analysis showed (correctly) that there was no phase transition in the model at any nonzero temperature. Ising also further speculated that the model would also fail to show a transition in three dimensions. This result was not particularly surprising, and could most likely be attributed to the oversimplifications inherent in the model being used. Somewhat in response to this result, Heisenberg posited his own, more sophisticated, model of spin interactions to attempt to explain ferromagnetism [12].

And so it was in this state that the model languished for nearly a decade, likely headed to obscurity, before Rudolph Peierls presented a simple argument purporting to show that in two dimensions the model was guaranteed to exhibit a phase transition at some critical temperature. This led to a re-examination of the model for understanding critical phenomena, and culminated in Lars Onsager's landmark solution of the two-dimensional model in zero external magnetic field in 1942. Onsager's approach was, in principle at least, very similar to Ising's; Find an analytical form for the partition function so that derivatives of the free energy can be examined. The technical difficulties were, however, considerably greater when moving from one to two dimensions, and the jump to three dimensions has proven so challenging that an analytical solution has yet to be found [13].

In part driven by these difficulties, as well as the advent of the computer, an increasing number of researchers turned towards numerical methods for further exploring the critical and cooperative behavior of the model. A common tool in this endeavor is the use of Monte Carlo simulations, where an ensemble of models are randomly evolved in time in a manner consistent with the underlying physics of the system. Each of these simulations represents a possible trajectory of the system through configuration space, and, with enough time and a large enough ensemble, will accurately reflect the actual distribution over the states. This approximate $P_{\mu}^{*}$ can then be used in lieu of constructing the partition function to measure average properties of the system.

To proceed in this manner assumes the existence of some dynamical process within the model, an aspect which was not present in Lenz's original formulation nor in typical textbook treatments. While the Ising Hamiltonian of eqn. 1.17 provides the energy for a given configuration of spins $\{\sigma\}$, it does not dictate the time evolution of the system. To make the model accessible to Monte Carlo simulation, as well as to probe beyond its static properties, dynamics need to be added to the model.

Promoting the model to a kinetic one, two common forms of dynamics are used: Glauber, spin-flip [14], or Kawasaki, spin exchange [15]. In the former, a spin is chosen at random, and flipped according to some probabilistic rule (which depends on $\mathcal{H}$ and $T^{1}$.), so that the total

\footnotetext{
${ }^{1}$ Other choices are also possible, as for example in the directed Ising model where the probabilistic rule
} 
magnetization, $M \equiv \Sigma_{i} \sigma_{i}$, fluctuates in time. By contrast, in the latter a random nearest neighbor pair of spins are chosen and exchanged. Thus, $M$ remains constant, a dynamics particularly suitable for describing, for example, binary alloys with spin \pm 1 representing, say, a mixture of $\mathrm{Cu}$ and $\mathrm{Zn}$. This version is also known as the Ising lattice gas [20], with spin \pm 1 referred to as particle and hole, a language which will be adopted predominately throughout the remainder of this study.

For an Ising system in thermal equilibrium at $T$, the rule governing the dynamics used must produce the appropriate Boltzmann distribution for $P_{\mu}^{*}$. A standard method by which to accomplish this is by setting the probabilistic rates for flips or exchanges via the Metropolis algorithm [21]. Since the dynamics are Markovian in nature, a reasonable stating point for determining these rates is with the master equation, eqn. 1.10. Based on the discussions in section 1.2, in equilibrium detailed balance, eqn. 1.14, is fulfilled. Combining this with the expression for the Boltzmann distribution, eqn. 1.7, we have the following relationship between the rates:

$$
\frac{W_{i}^{j}}{W_{j}^{i}}=e^{-\Delta \mathcal{H} / k_{B} T},
$$

where $\Delta \mathcal{H}=\mathcal{H}(i)-\mathcal{H}(j)$. The rates are determined absolutely by choosing the transition for which $\Delta \mathcal{H}<0$ to one. By the ratio above, this automatically sets the inverse rate. This choice of rates can be concisely denoted as $\min \left[1, e^{-\Delta \mathcal{H} / k_{B} T}\right]$.

\subsection{Non-equilibrium Ising models}

The previous section demonstrated the numerous ways, both analytical and numerical, in which the Ising model has been studied in the years subsequent to its creation. Implicit in all these methods was the assumption that the model was held in equilibrium with some thermal reservoir at a fixed temperature $T$. As discussed in section 1.2, once this constraint is lifted and a broader range of non-equilibrium systems are examined, a host of new techniques are required to hope to understand the unique, and often bewildering, behavior of NESS.

In this spirit, many studies were conducted for the paradigmatic Ising model with nearest neighbor interactions, driven to NESS by a variety of mechanisms. In all cases, the key lies in coupling the system to two (or more) energy reservoirs, such as a bath and an external drive, or two thermal baths at different temperatures. In general, there is a stationary net flux of energy through the system, from say, the hotter bath to the cooler one, as the system settles into a NESS. Over the last three decades, a wealth of surprising phenomena associated with such NESS have been discovered, many of which remain poorly understood. One general rule gleaned so far is that, while driving a system into a NESS will produce novel

depends unequally on the neighbors of the flipping spin, see [16-19] 
effects in general, the most dramatic differences tend to emerge when the system is endowed with a conservation law (e.g., particle conservation). For example, taking a model with spin-flip dynamics out of equilibrium produces observable effects, but the critical properties, say, remain in the equilibrium universality class [22-25], i.e. exhibit the same universal critical behaviors as the standard equilibrium Ising model. To further explore the impact of non-equilibrium effects on a conserved system, this study is devoted to an Ising system with a conservation law in arguably the simplest of settings: the lattice gas on a ring (onedimensional periodic lattice) coupled to two temperature reservoirs.

As a final step before delving into our specific system, we will provide a brief overview of the various ways in which the Ising model has been coupled to two thermal baths and the NESS behavior that emerged. Such a discussion will be helpful when encountering the term "two-temperature Ising model" in the literature, as well as being useful for distinguishing previous approaches from those of the present study. Since the Ising model was not originally imagined to study non-equilibrium systems, if we insist on coupling our system to two T's, then it is clear that there is an enormous variety of ways to implement this. The brief survey below demonstrates a number of ways in which this has been attempted and provides further context for our study.

Although spin-flip rates which depend on two temperatures were introduced as early as 1982 [26], the bulk of such explorations was carried out in the 90's (see, e.g., [27] for a review of these early studies). Nearly all studies involve dynamics which are (essentially) translationally invariant, while many involve anisotropy. There are a number of examples of work being done on systems coupling the two baths to every spin or every other spin (for Glauber dynamics in $d \geq 1$ ) [28-36]. In the case of the alternating coupling, the two dimensional model was found to exhibit an order-disorder phase transition that belonged to the Ising universality class. For the one-dimensional model, an exact expression for the correlations between the spins was found, indicating that there is no phase transition in this case. So while the nonuniversal properties of this system, such as the exact distribution function over the states, are modified, many of the critical behaviors remain unchanged.

Other models performed the coupling of the two reservoirs by introducing anisotropic exchanges of pairs in the $x$ or $y$ directions (in $d=2$ ) [37-41], where each axis of the lattice satisfies detailed balance at its own unique temperature. Here again, the universal properties of the critical point are found to fall within the Ising universality class. Even more exotic models involved a mixture of Glauber dynamics at one $T$ and Kawasaki at another $T$ [42-45]. In all cases, though the presence of two coupled reservoirs continuously drives energy through the system even in the steady state, the model remains spatially homogeneous.

By contrast, our study will focus on inhomogeneous couplings: one entire region of the system coupled to one bath and the complement coupled to the other bath. Recent efforts were directed towards (a) Glauber dynamics on two semi-infinite chains $(d=1)$, coupled to two baths and joined at the ends $[46,47]$ and (b) Kawasaki exchange on two halves of a finite $d=2$ system $[48,49]$. While exact analytic results are available for the former, the novelties 
of the NESS can be expected. For the latter, we have only simulation results, which provided more exotic and surprising behavior, e.g., convection cells and states with multiple strips. Naively, the contrast between two sets of results might be attributed to the difference in $d$ : the absence/presence of a phase transition. Yet, the other difference, Glauber vs. Kawasaki dynamics, may be more crucial. It is in this context that we conduct the present study: an Ising chain with spin-exchange dynamics, coupled to two temperatures.

\subsection{Organization of this study}

The rest of this study is organized as follows. In the next section, we present a detailed description of the model. Although the static, equilibrium properties of the standard Ising chain can be found in most textbooks, two aspects - Kawasaki dynamics and fixed $M$ ensemble - are less well known and will be discussed in Section 3. We also present some results for an equilibrium model where different coupling constants are used in different sectors of the system, as well as performing a detailed analysis using the master equation to find the Boltzmann distribution for smaller system sizes. In the final Section 4, we present the many surprising phenomena discovered through simulations of the two-temperature model and compare these results with those obtained for the equilibrium models considered in Section 3. We conclude with a summary. Some technical details are provided in Appendices. 


\section{Chapter 2}

\section{Model Description}

This chapter is a reproduction of the model description found in [1], with additional discussions from the appendix of the same paper. Reprinted excerpts with permission from N.Borchers, M. Pleimling, and R.K.P. Zia, Physical Review E, 90(6):062113, 2014. Copyright 2014 by the American Physical Society.

We consider the standard Ising system on a ring of spins, $\sigma_{i}$, at $i=1, \ldots, L$ sites with nearest neighbor, ferromagnetic interactions. Here, we provide a detailed description of how we couple this system to two thermal baths, in terms of what is implemented in our simulations.

First, we evolve a configuration by Kawasaki exchange with Metropolis rates [21]: At each time step (attempt), choose a random pair of nearest neighbor spins and exchange them with the following probability. Clearly, a non-trivial update must involve two spins that are opposite. If $\Delta \mathcal{H}$ denotes the change in $\mathcal{H}$ due to the exchange, then we allow it to take place with probability $\min \left[1, e^{-\Delta \mathcal{H} / k_{B} T}\right]$, where $T$ is the temperature of the thermal bath. Thus, the overall magnetization of the system, $M$, is conserved. Since there is no phase transition in the standard one-dimensional Ising model, it is natural for us to restrict ourselves to systems with $M=0$. Of course, these systems will settle into an equilibrium state associated with the $M=0$ ensemble.

Our goal is to explore NESS, associated with a dynamics which violates detailed balance. One natural way is to couple two sectors of the ring to reservoirs of differing temperatures. Specifically, exchanges within a 'window' of length $w$ will be updated with temperature $T_{w}$, while the rest of the ring will be coupled as above. Obviously, the system will revert back to an equilibrium Ising model for $T_{w}=T$ or $w=0$. To simplify our study, instead of exploring the full control space of $T-T_{w}$, we set $T_{w}=\infty$ in the following. With Metropolis rates, this choice implies that all attempts at exchanging pairs within the window are successful, regardless of $\Delta \mathcal{H}$. One question naturally arises: How do such exchanges differ from the case involving $J=0$, i.e., non-interacting spins? We will address this subtle issue at the end 
of this section, along with a crucial discussion of detailed balance violation.

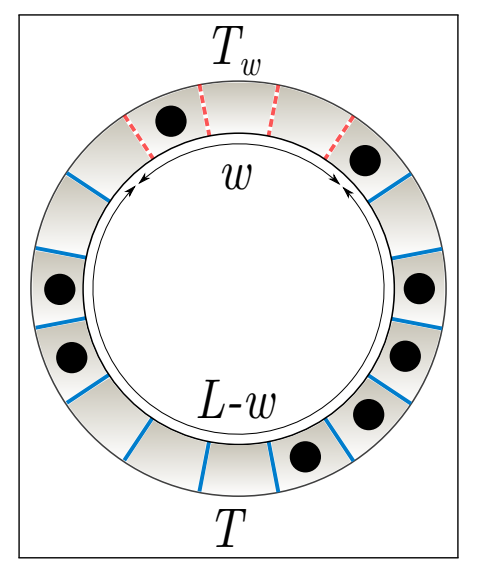

Figure 2.1: Sketch of the two-temperature Ising ring model studied in this paper. Whereas exchanges across blue borders are accepted with the ordinary Metropolis rate for a system at temperature $T$, exchanges across red borders are accepted with the corresponding rate for a system at temperature $T_{w}$. Reprinted figure with permission from N.Borchers, M. Pleimling, and R.K.P. Zia, Physical Review E, 90(6):062113, 2014. Copyright 2014 by the American Physical Society.

How these rates operate is concisely captured in Fig. 2.1, where the lattice is depicted as being half-filled with particles/holes (corresponding to $M=0$ ). Any exchange takes place across a 'border' between two adjacent sites. Of the $L$ borders, we color $w$ of them red and the rest blue. An exchange across a dashed red border always takes place. By contrast, to exchange a pair across a blue border, $\Delta \mathcal{H}$ must be computed. Then the exchange is allowed with probability $\min \left[1, e^{-\Delta \mathcal{H} / T}\right]$ (i.e., the rate used to study the ordinary equilibrium Ising model at $T)$. In the following, we will refer to this as the $2 T$ model, short for 'twotemperature Ising model.' Let us emphasize that all properties of this system are embodied in a time dependent distribution, $P[\{\sigma\} ; t]$, governed by a simple master equation:

$$
P\left[\left\{\sigma^{\prime}\right\} ; t+1\right]=\sum_{\{\sigma\}} W\left[\{\sigma\},\left\{\sigma^{\prime}\right\}\right] P[\{\sigma\} ; t]
$$

where $W$ is the transition probability from $\{\sigma\}$ to $\left\{\sigma^{\prime}\right\}$ in one attempt:

$$
\begin{array}{r}
L^{-1} \sum_{i} \Delta_{i}\left[\left\{1-q_{i} \delta\left(\sigma_{i-1}-\sigma_{i}\right) \delta\left(\sigma_{i+1}-\sigma_{i+2}\right)\right\} \delta\left(\sigma_{i}^{\prime}+\sigma_{i}\right) \delta\left(\sigma_{i+1}^{\prime}+\sigma_{i+1}\right)\right. \\
\left.+q_{i} \delta\left(\sigma_{i-1}-\sigma_{i}\right) \delta\left(\sigma_{i+1}-\sigma_{i+2}\right) \delta\left(\sigma_{i}^{\prime}-\sigma_{i}\right) \delta\left(\sigma_{i+1}^{\prime}-\sigma_{i+1}\right)\right]
\end{array}
$$

Here, $\delta$ is the Kronecker delta (i.e., unity if its argument vanishes and zero otherwise), $\Delta_{i} \equiv \delta\left(\sigma_{i}+\sigma_{i+1}\right) \Pi_{k \neq i, i+1} \delta\left(\sigma_{k}^{\prime}-\sigma_{k}\right)$ ensures that only the pair $\sigma_{i}, \sigma_{i+1}$ may change, and 


$$
q_{i}=1-e^{-4 J / k_{B} T(i)}
$$

with $T(i)=T_{w}$ for $i=1, \ldots, w$ and $T(i)=T$ for $i=w+1, \ldots, L$, is the probability that this pair is unchanged. Note that $\sum_{\left\{\sigma^{\prime}\right\}} W\left[\left\{\sigma^{\prime}\right\},\{\sigma\}\right]=1$ so that $P$ remains normalized at all times. It can be shown that, as $t \rightarrow \infty, P$ settles into a unique stationary distribution, $P^{*}[\{\sigma\}]$, which is very different from the Boltzmann $P_{e q}$ (with any $T$ ). Indeed, for small systems $(L=6,8)$, we found explicit distributions to have very different degeneracy structures than the equilibrium distributions for the same $\mathcal{H}$. These distributions will be considered in a later section. For reasonably large systems (e.g., $L \gtrsim 50$ ), finding these $P^{*}$ 's is impractical numerically and analytically (due to detailed balance violation), let alone computing averages of observables from $P^{*}$. Thus, Monte Carlo simulations are the only viable technique for us to make progress ${ }^{1}$.

When we consider the exchanges away from the interface between the two sectors, the rates resemble those for an inhomogeneous Ising model (with $J=0$ inside the window) coupled to a single reservoir at temperature $T$. Specifically, we can still exploit Fig. 2.1, by regarding the blue borders as energy bonds of strength $J$ and associating the red ones with $J=0$. Let us refer to this model as the $2 J$ model. Clearly, the stationary distribution here is just $\exp \left[-J \sum_{i} \sigma_{i} \sigma_{i+1} / k_{B} T\right]$, where the sum is over only the blue bonds. Its statistical properties are just as accessible as the standard model. What is the key difference between the transition rates for this model and the $2 T$ case? It lies in the exchanges of just two pairs of spins at each interface. To see this mathematically, we note that the dynamics needed for a generally inhomogeneous Ising model (i.e., $\mathcal{H}=-\Sigma_{i} J(i) \sigma_{i} \sigma_{i+1}$ ) in contact with a single bath are the same as above, except for

$$
q_{i}=1-e^{-2(J(i-1)+J(i+1)) / k_{B} T}
$$

instead of Eq. (2.3). For the $2 J$ model, we have $J(i)=0$ and $J$, for $i \in[1, w]$ and $[w+1, L]$ respectively. How does one set of rates obey detailed balance and the other set violate it?

To emphasize this disparity in rates, which is the crucial difference between the $2 T$ and the $2 J$ model, we will illustrate detailed balance violation in the former with "irreversible" cycles of configurations (Fig. 2.2a,b). By contrast, the same cycles in the $2 J$ model is reversible (Fig. 2.2c,d). To make this distinction clear, we added a notation for the bond energies here, i.e., presence or absence of a horizontal dash referring to the ordinary ferromagnetic $J=1$ or the free $J=0$ interactions between nearest neighbors, respectively. Meanwhile, the vertical lines between neighbors carry the same meaning as in previous sections, i.e., exchanges across solid blue lines being updated with the finite $T$ and those across dashed red lines being updated with $T=\infty$.

Consider first the configurations in Fig. 2.2a, arranged around the quarters of a clock face.

\footnotetext{
${ }^{1}$ In the following we express energies in units of $J$, whereas temperatures are expressed in units of $J / k_{B}$.
} 


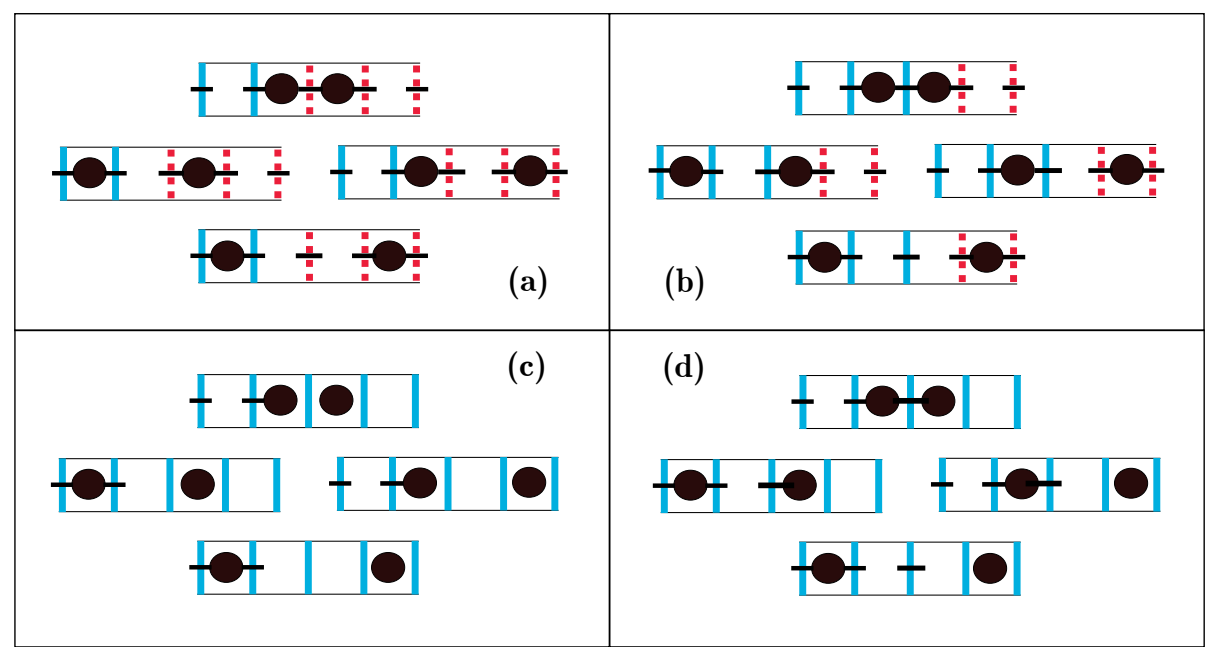

Figure 2.2: Two cycles of configurations illustrating irreversibility and detailed balance violation in the $2 T$ model $(\mathrm{a}, \mathrm{b})$. By comparison, these cycles are reversible in the $2 J$ model $(\mathrm{c}, \mathrm{d})$. See text for explanation of symbols. Reprinted figure with permission from N.Borchers, M. Pleimling, and R.K.P. Zia, Physical Review E, 90(6):062113, 2014. Copyright 2014 by the American Physical Society.

For simplicity, suppose there are no particles outside the four sites shown. The $2 T$ model is notable for the fact that exchanges across different vertical lines are controlled by thermal baths at different temperatures. For the clockwise cycle beginning at 12 o'clock, the product of the transition probabilities is simply $1 \times 1 \times 1 \times 1=1$. The first transition, in which the bonded particles are broken apart, occurs with probability one because this transition involves the rightmost particle moving across a red boundary at infinite temperature. For the counter-clockwise cycle, the probability is $e^{-1 / T} \times 1 \times 1 \times 1$, which is not unity except for $T=\infty$. The first step of the cycle, in which the bonded particles are broken apart, is again critical. Here the leftmost particle moves across a blue boundary, and hence is controlled by the lower temperature reservoir. It is for this reason that the probability of this cycle picks up the Boltzmann factor, and this reversed cycle therefore occurs less frequently than the former. This explicitly breaks time symmetry, i.e. the direction of time can be estimated by which cycle is observed, but such arguments only hold near the boundary of the two regions. The same inequality holds for the cycles involving the configurations in Fig. 2.2b. The inequality of these products is a hallmark of detailed balance violation [50] and signals a presence of a non-equilibrium stationary state [7]. By stark contrast, it is straightforward to check that the products around the cycles in Figs. 2.2c,d, are the same as the products for the reversed cycles. Of course, this equality necessarily holds for a system in thermal equilibrium, governed by the Hamiltonian $-J \Sigma_{i} \sigma_{i} \sigma_{i+1}$ (with appropriate $J=1,0$ ).

To end this section, let us provide the details of our simulation methods. Starting with a random configuration of spins with $M=0$ if $L$ is even $(M=1$ for odd $L)$, we randomly choose a pair of spins and update them according to the probabilities in Eq. (2.1). A Monte 
Carlo step (MCS) is defined as $L$ such attempts. Whereas our primary concern is with the steady state properties, we also need to ensure that the system has relaxed sufficiently into the NESS. For this purpose, we also collected data on the transient regime. After the relaxation stage, a suitable number of simulation steps is used to measure the averages of various observables. Specifically, we will focus on two-spin correlations

$$
G(i ; r) \equiv\left\langle\sigma_{i} \sigma_{i+r}\right\rangle
$$

(for certain $i$ 's), the total magnetization within the window

$$
M_{w} \equiv \sum_{i=1}^{w} \sigma_{i}
$$

the distribution of the normalized window magnetization $m \equiv M_{w} / w$

$$
\mathcal{P}_{w}(m) \equiv \sum_{\{\sigma\}} \delta\left(m-\sum_{i=1}^{w} \sigma_{i} / w\right) P^{*}[\{\sigma\}],
$$

the energy density profile

$$
u_{i} \equiv-J\left\langle\sigma_{i} \sigma_{i+1}\right\rangle=-J G(i ; 1)
$$

and its sum $\Sigma_{i=1}^{L} u_{i}$ (i.e., $\left.\langle\mathcal{H}\rangle\right)$. The number of relaxation steps required varies considerably with the system parameters and increases rapidly for larger $L$ 's and smaller $T$ 's, see the discussion below. Finally, we performed typically 40 to 100 independent runs (with different initial conditions), so that time-dependent quantities are constructed by averaging over these runs. Of course, for stationary state properties, we perform both a time and ensemble average. In the remainder of this study, we choose units such that $J / k_{B}=1$. 


\section{Chapter 3}

\section{The equilibrium Ising lattice gas}

The remaining sections of this study are adapted from the results of [1]. Additional results concerning small systems and the nature of the $2 T$ steady state are also included. Reprinted excerpts with permission from N.Borchers, M. Pleimling, and R.K.P. Zia, Physical Review E, 90(6):062113, 2014. Copyright 2014 by the American Physical Society.

Before reporting results on the $2 T$ Ising ring, we will briefly review the relevant properties of two equilibrium cases, namely the standard homogeneous Ising model with uniform couplings as well as the $2 J$ model discussed in the previous section.

\subsection{Standard homogeneous Ising model}

Though the standard Ising chain is a textbook model, we present some less well-known aspects, so as to facilitate the comparison with its non-equilibrium counterpart. Deferring technical details to Appendix A, we report only simulation results here.

As the system relaxes into the stationary state, its behavior is dominated by eigenvectors which are associated with eigenvalues of $W$ (see Eq. (2.2)) close to unity. While the details depend on the specifics of the rates, it is known that a power law ( $t^{1 / 3}$ law) can be expected with Kawasaki dynamics. In particular, at low temperatures domains (of the same spin) should form and grow in size. Of course, unlike typical coarsening behavior of a system with spontaneous magnetization (i.e., below critical temperature), domains in the ring cannot be much larger than the correlation length: $O\left(e^{1 / T}\right)$. Nevertheless, in the growing regime, their sizes scale with $t^{1 / 3}[51]$. Though we did not measure domain sizes, the relaxation of all the quantities we study are consistent with this law.

The quantity of primary interest for evaluating the rate of relaxation will be the average energy density $\langle\mathcal{H}\rangle / L$. This quantity is closely related to domain growth since in one dimension the overall energy of the system depends directly on the number of these domains. 


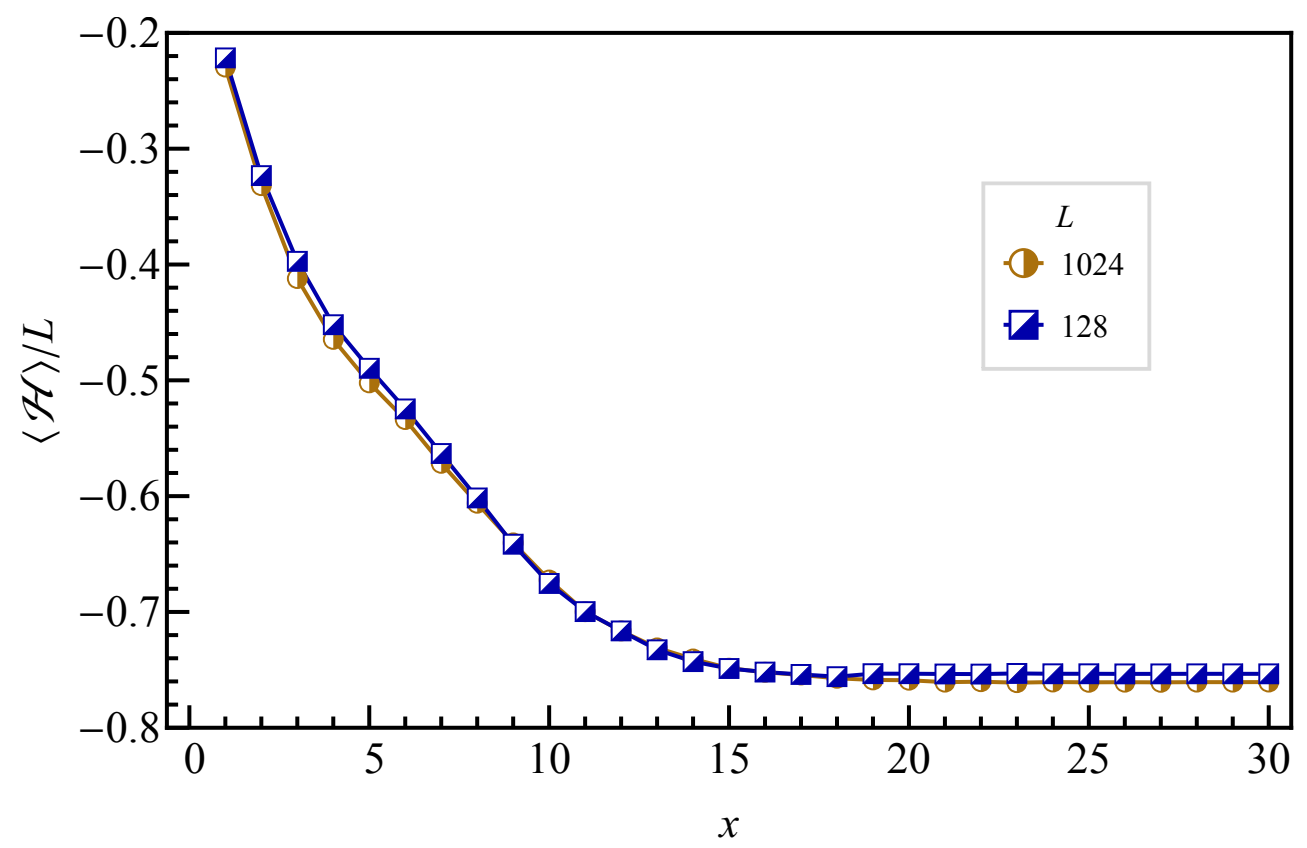

Figure 3.1: Log-binning relaxation of the energy density $\langle\mathcal{H}\rangle / L$ for the standard Ising system at temperature $T=1$ and two different lattice sizes $L$. In this plot (as well as in the other figures below showing log-binning relaxation) the average of the quantity of interest is sampled at intervals between $2^{x}$ and $2^{x+1}$ MCS. The equilibrium value of $\langle\mathcal{H}\rangle / L$ is already very close to the value $-\tanh (1) \approx-0.76$ of the infinite lattice. The data results from averaging over an ensemble of 40 to 100 independent realizations. Error bars are comparable to the sizes of the symbols. Reprinted figure with permission from N.Borchers, M. Pleimling, and R.K.P. Zia, Physical Review E, 90(6):062113, 2014. Copyright 2014 by the American Physical Society.

In addition, the conservation law ensures the average size of these domains must be related to their overall number. Fig. 3.1 shows how the energy density relaxes for different $L$ 's via the log-binning procedure, a technique in which the averages of quantities of interest are sampled at intervals between $2^{x}$ and $2^{x+1}$ MCS. Of particular note here is that finite-size effects have only a minor influence on the relaxation of the energy density, as the curves here are nearly identical. The rate of relaxation is therefore entirely determined by the dynamics of the system.

In Fig. 3.2, we show the effects of different $T$ on the relaxation of $\langle\mathcal{H}\rangle / L$. Not surprisingly, the energy of a system coupled to lower temperatures takes considerably longer to settle. For our $2 T$ model, nothing was known about such relaxation times and it was therefore 


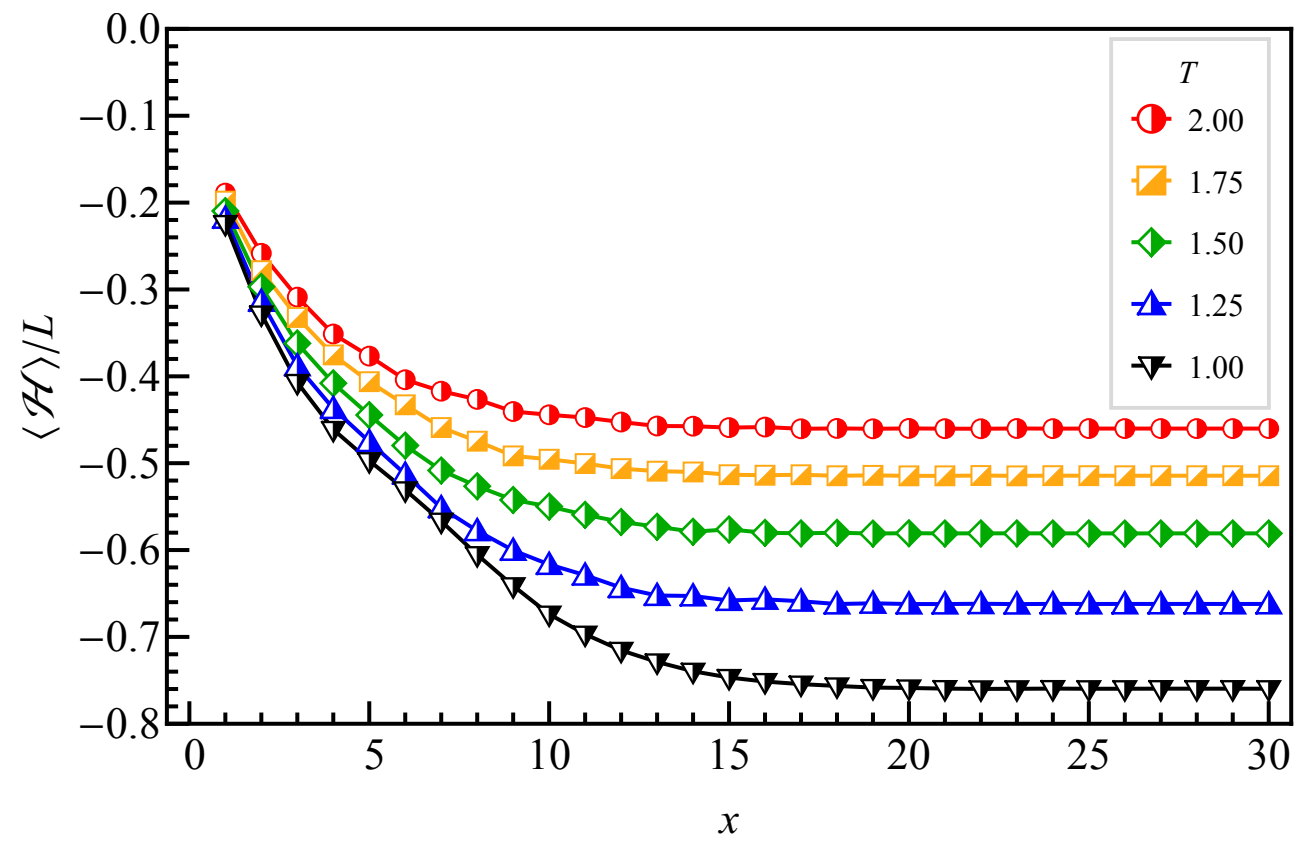

Figure 3.2: Log-binning relaxation of the energy density for the standard Ising model with $L=512$. The data results from averaging over an ensemble of 40 to 100 independent realizations. Error bars are comparable to the sizes of the symbols. Reprinted figure with permission from N.Borchers, M. Pleimling, and R.K.P. Zia, Physical Review E, 90(6):062113, 2014. Copyright 2014 by the American Physical Society.

critical to develop an approach to explore the evolution of the system towards the steady state. Below, we will use a similar approach to determine if those systems have settled into their NESS.

Ultimately, the utility of this procedure is that it provides a quantifiable measure for entrance into the steady state in that the initiation of the steady state can be identified with the beginning of the flat region of the corresponding log-binning curve. Unfortunately, there is an obvious issue with this approach; Simulation data is never truly flat, but only roughly so within some acceptable tolerance. The magnitude of this tolerance is essentially arbitrary, and in this way there is an unavoidable uncertainty in the time it takes the system to enter the steady state. There is no issue in making statements about relative relaxation times, as, for instance, was done concerning the curves in Fig. 3.2, but absolute measurements will be inherently imprecise. This issue can be resolved by beginning the system in both ordered and disordered states, and looking for the convergence of the log-binning curves. This ensures that the influence of all eigenvectors, save for the one associated with the unit eigenvalue, 
has decayed away sufficiently and that only $P^{*}$ remains. This approach is demonstrated in Fig. 3.3.

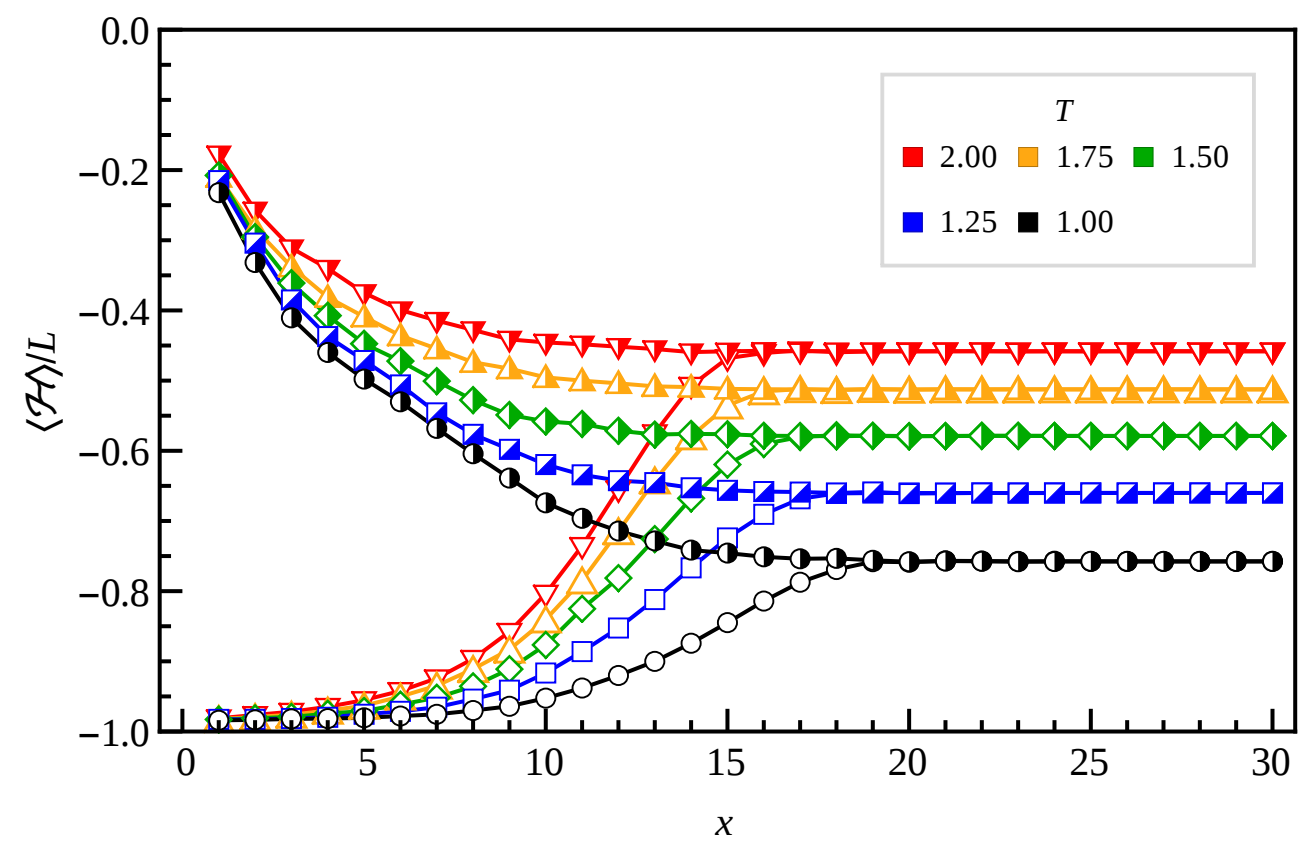

Figure 3.3: Convergence of the energy density log-binning for the standard Ising model with $L=256$. The data results from averaging over an ensemble of 50 independent realizations. Filled symbols are for random initial conditions, while empty symbols are for ordered initial conditions. Error bars are comparable to the sizes of the symbols.

Turning to properties in the stationary state, let us first illustrate a typical space-time plot of the configurations, in Fig. 3.4, with a system with $L=128$ set at $T=1$. A black/white square denotes a particle/hole. Each row is a snap shot of the ring, while successive rows are separated by 10 MCS. The presence of semi-persistent domains is evident. Of course, they shrink and grow randomly, via evaporation and condensation at the edges, resulting in, at times, apparent drifts like a random walker.

As mentioned previously, the growth of these domains should not be confused with the coarsening found in systems with spontaneous magnetization. This difference is abundantly clear when viewing the behavior of these domains over longer time-scales. In Fig. 3.5 a space-time plot is shown where the system is sampled at exponentially increasing intervals to reveal the qualitative behavior for a single simulation across many time scales. The system is initially sampled every MCS, and therefore the phase-separated domains appear relatively stable and are relatively fixed in position as time moves forward. After 32 samples taken in 
such a way, the sample rate is halved, and a sample is now taken only every other MCS. This is continued in this way, halving the sample rate after every 32 samples, until a total of $2^{25}$ MCS have been performed. By the end of the run, there are $2^{20}$ samples between adjacent horizontal lines. In this way, a time equivalent to the entire proceeding time displayed within the plot is now compressed within a single row of the plot! The impermanence of these domains is clear by the end of the plot, as there are no stable structures when viewed at these time scales, and it is clear that the system remains in a fully disordered state.

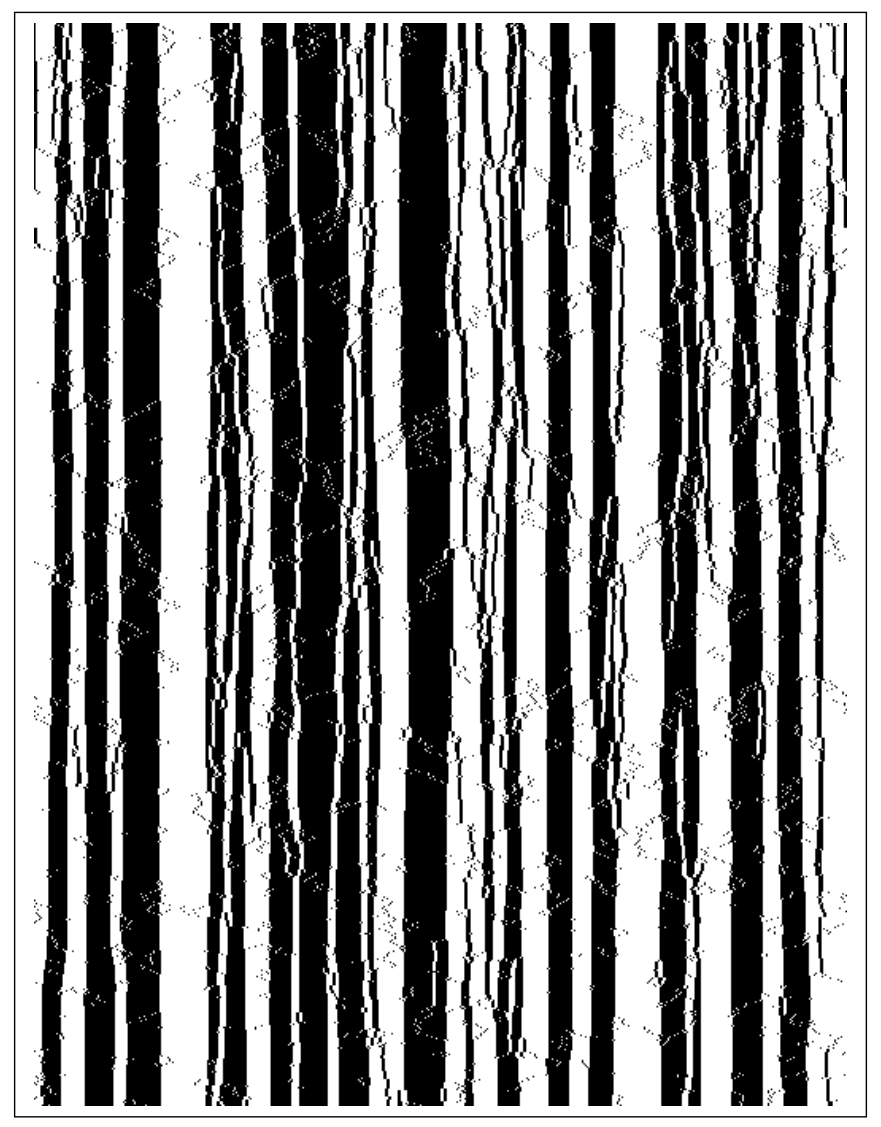

Figure 3.4: Space-time plot of the standard Ising system after it has reached equilibrium at $T=1$. The plot shows the evolution of the system over a total of $2^{12}$ MCS after an initial relaxation period of $2^{15} \mathrm{MCS}$, where two consecutive horizontal lines are separated by 6 MCS. The system size is $L=512$.

These figures should provide an intuitive picture for the quantitative aspects, such as the twospin correlations $G(r) \equiv G(i ; r)$ (for a particular $i$, but independent of $i$ due to translational invariance) and distributions $\mathcal{P}_{w}(m)$. The former is a standard quantity of interest in the study of statistical mechanics. By contrast, we are not aware of systematic investigations of the latter. Here, $m$ is a local magnetization, coarse-grained at the level of $w$, so that $\mathcal{P}_{w}(m)$ provides information on clustering at this length scale. Of course, at the level of the entire system, $\mathcal{P}(M)$ (for the non-conserved case) enjoyed considerable attention, since it signals 


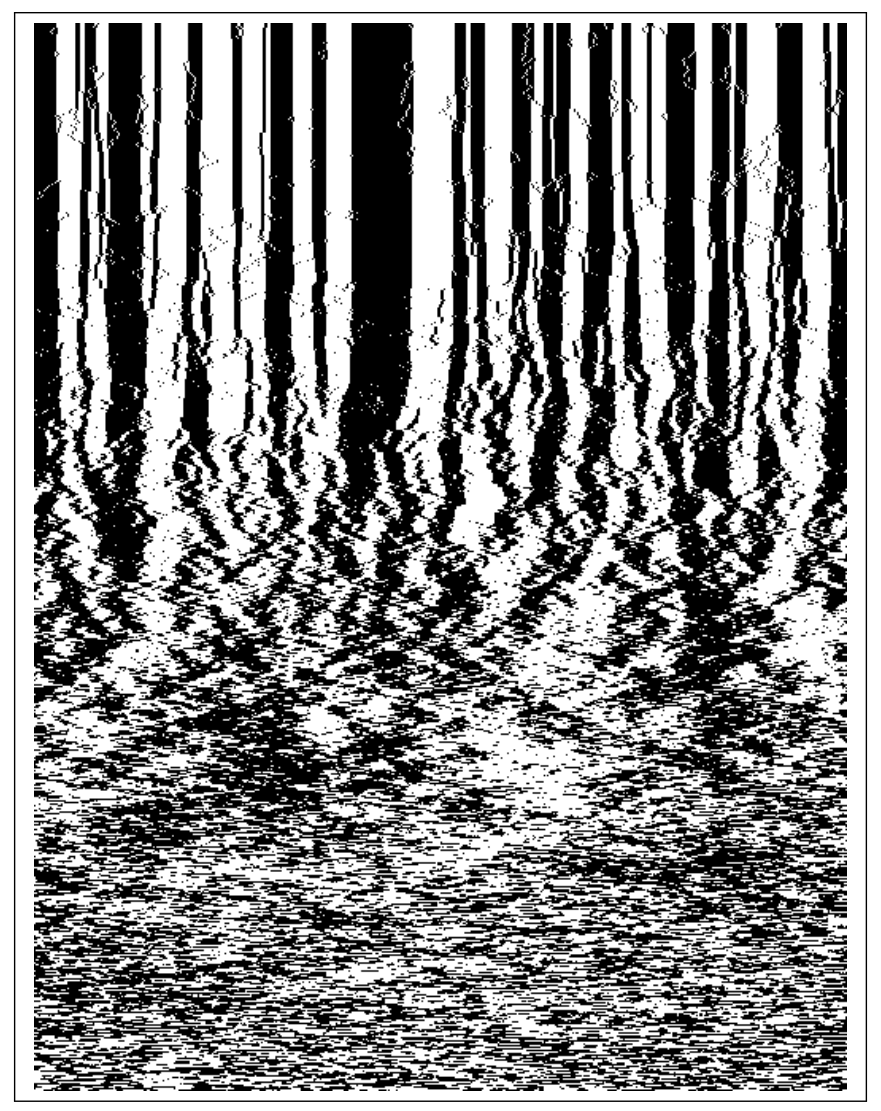

Figure 3.5: Logarithmic space-time plot of the standard Ising system after it has reached equilibrium at $T=1$. The plot shows the evolution of the system over a total of $2^{25}$ MCS after an initial relaxation period of $2^{15}$ MCS. Here, the number of MCS between adjacent horizontal lines increases exponentially as the time proceeds downwards. Initially, each line corresponds to a single MCS, but by the end there are $2^{20}$ MCS between adjacent lines. The system size is $L=512$.

the onset of long range order and reveals non-trivial critical properties (for Ising models in $d>1$ ). Since we will focus on $\mathcal{P}_{w}(m)$ for the $2 T$ model, collecting data on its equilibrium counterpart will provide both a good baseline and a sharp contrast.

For a non-conserved Ising model on a ring, $G$ is well-established:

$$
G(r)=\left(\omega^{r}+\omega^{L-r}\right) /\left(1+\omega^{L}\right)
$$

where

$$
\omega \equiv \tanh (1 / T)
$$




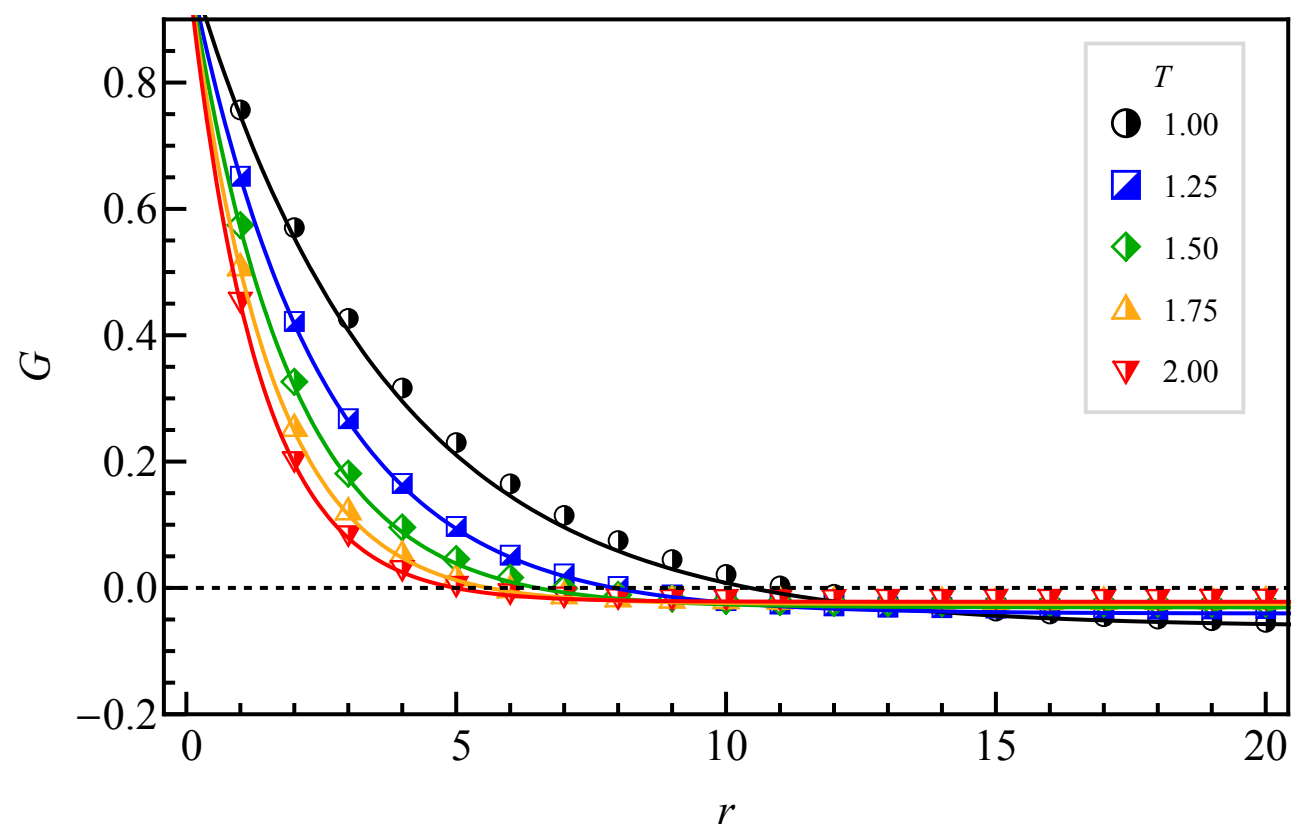

Figure 3.6: $G(r)$ for the standard equilibrium Ising ring with Kawasaki dynamics for a system of length $L=127$. L is chosen to be odd to ensure a symmetric result about a given reference point. The lines refer to the approximation Eq. (3.3). Reprinted figure with permission from N.Borchers, M. Pleimling, and R.K.P. Zia, Physical Review E, 90(6):062113, 2014. Copyright 2014 by the American Physical Society.

Of course, $G>0$ for all finite $T$. But the $M=0$ constraint adds complications, since $\Sigma_{r} G(i ; r)=0$ in this case. With details deferred to Appendix B, we find that a simple linear approximation

$$
G \cong A\left(\omega^{r}+\omega^{L-r}\right)+B
$$

(with $A$ and $B$ fixed by $G(0)=1$ and $\Sigma G=0$ ) agrees quite well with data. In Fig. 3.6, we illustrate this agreement in a small system with $L=127$ sites. There are no surprises here; its main purpose is for comparison with Fig. 4.10 below.

Turning to $\mathcal{P}_{w}(m)$, we illustrate with Fig. 3.7 the case of $L=128$ and $w=32$. For each $T$, we compile a histogram from $\sim 10^{8}$ measurements of $M_{w}$. Not surprisingly, every distribution is peaked at $m=0$, the signature of disorder. In stark contrast, below we will find transitions to bi-modal distributions in the $2 T$ model, shown in Fig. 4.12. 


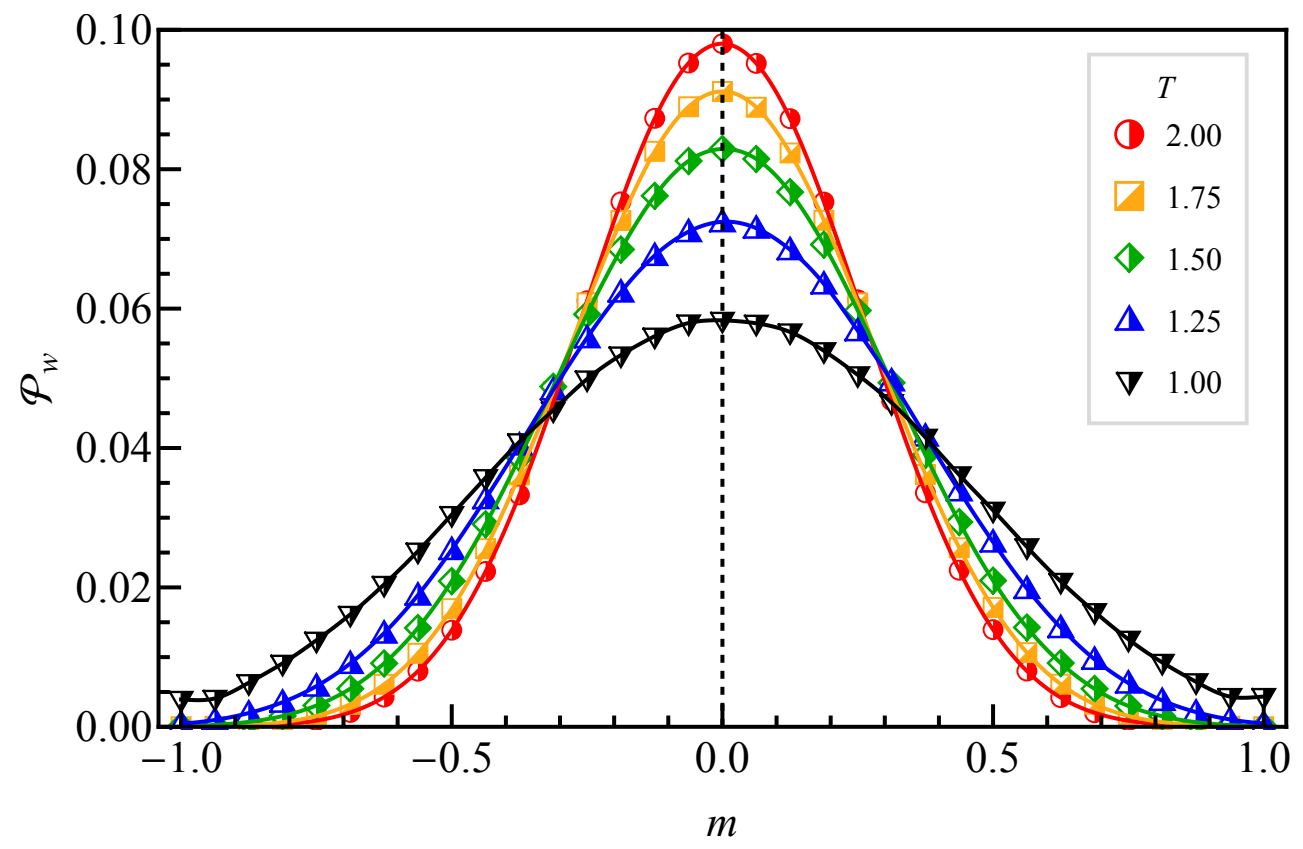

Figure 3.7: Equilibrium distributions of the normalized window magnetization $m$ for the standard Ising model with $L=128$ and $w=32$. Reprinted figure with permission from N.Borchers, M. Pleimling, and R.K.P. Zia, Physical Review E, 90(6):062113, 2014. Copyright 2014 by the American Physical Society.

\subsection{The 2J model}

While the above discussion captures many of the relevant features of the equilibrium Ising model in one dimension, it is worth briefly considering another equilibrium model, namely the $2 \mathrm{~J}$ model. The $2 \mathrm{~J}$ model is distinguished from the standard model in that there exists a window (i.e. region) of width $w$ embedded in the lattice where the coupling constant vanishes. The remainder of the $L-w$ sites outside of that window retains the standard coupling constant of $J$. While this change does not alter the equilibrium Boltzmann statistics which govern the behavior of this model, it does break the translational invariance present in the standard model in the same manner as the $2 T$ system does. As discussed in the previous Section, the sole difference between the $2 J$ and the $2 T$ models are the transition rates for exchanges of pairs of spins at the interface. For that reason, comparison between results obtained from the $2 J$ and $2 T$ models will be very enlightening.

Fig. 3.8 shows space-time plots for the $2 J$ model after having reached equilibrium. There are two clearly distinct regions which are immediately identifiable; the region inside the window which is disordered due to the absence of couplings between spins in that region, and the 

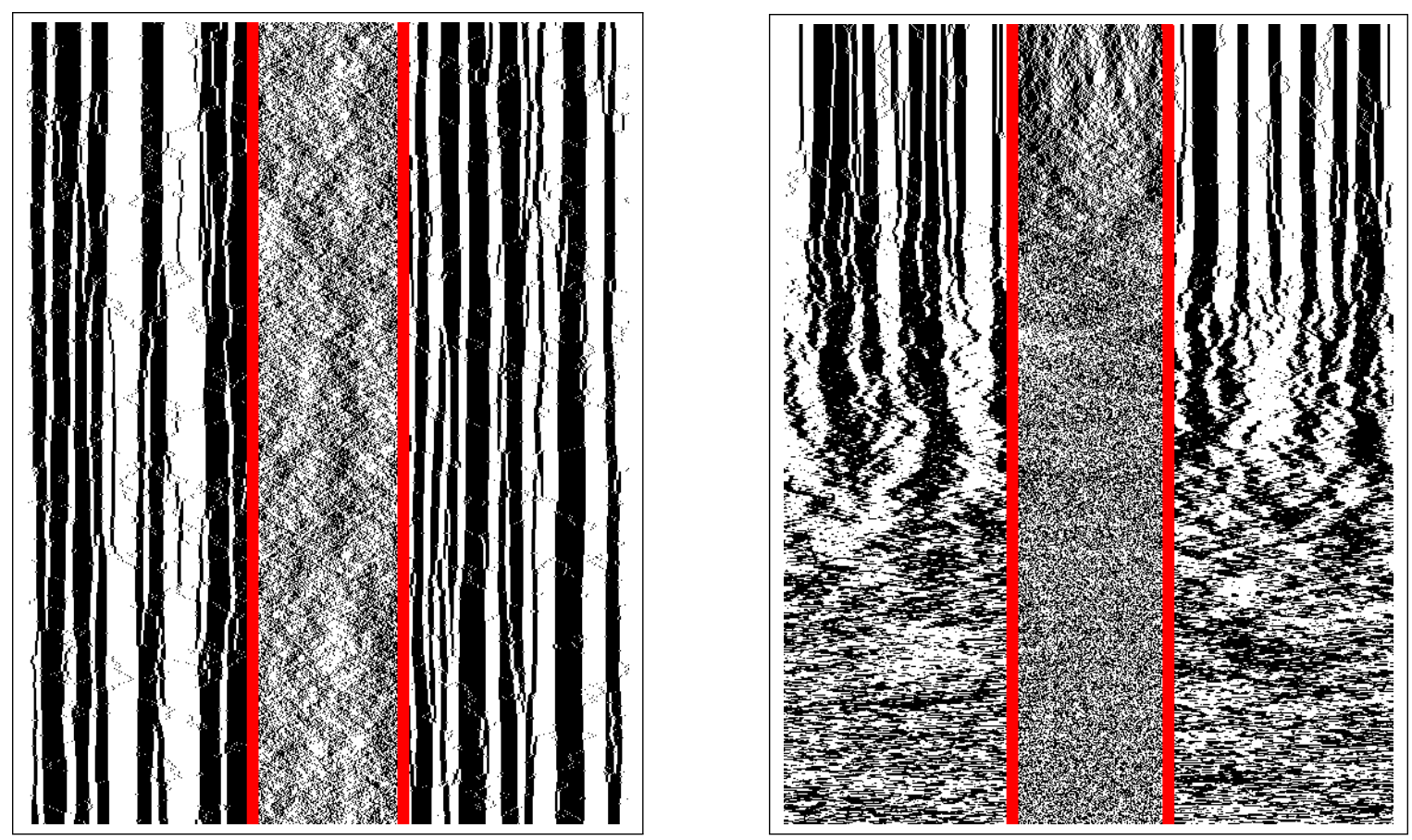

Figure 3.8: Space-time plots of the $2 J$ model after it has reached equilibrium at $T=1$. The left plot shows the evolution of the system over a total of $2^{12}$ MCS after an initial relaxation period of $2^{15}$ MCS, where two consecutive horizontal lines are separated by $6 \mathrm{MCS}$. The system size is $L=512$ and $w=128$. The right plot is the logarithmic version of this plot over $2^{25}$ MCS. The boundaries between the regions are highlighted in red.

region outside with semi-persistent domains similar to what is observed in the standard Ising model, see Fig. 3.4. As the presence of the interface does not create long-range correlations, the behavior of the system outside of the window is largely unaffected by the presence of the disordered sector.

Equilibrium distributions of the normalized window magnetization are displayed in Fig. 3.9 for the $2 J$ model. As expected for a disordered section, these distributions are Gaussian and only show a very weak dependence on the temperature of the system.

\subsection{Small system analysis}

Before moving on to the $2 T$ model which is at the heart of this study, it is worth considering some in depth analysis of small versions of the equilibrium models already discussed. Our focus in this section will be on $L=6$ in particular, as this represents the smallest lattice in which we can embed a window of various sizes for the $2 \mathrm{~J}$ model. While it is fully expected 


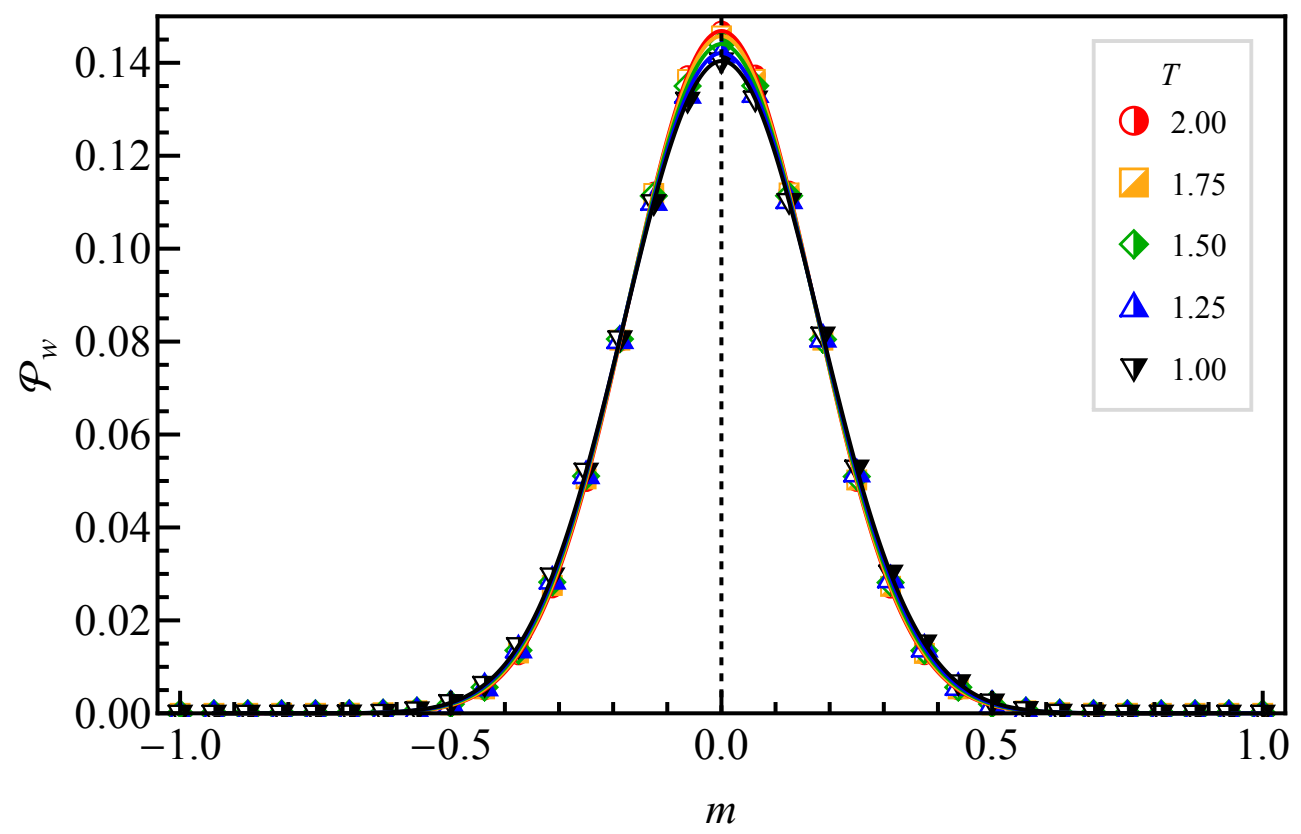

Figure 3.9: Equilibrium distributions of the normalized window magnetization $m$ for the $2 J$ Ising model with $L=128$ and $w=32$. Distributions for different temperatures are shown. Reprinted figure with permission from N.Borchers, M. Pleimling, and R.K.P. Zia, Physical Review E, 90(6):062113, 2014. Copyright 2014 by the American Physical Society.

that finite-size effects will dominate the behavior of such a small system, systems of this size present a unique opportunity to successfully perform analytical calculations. Though the insight that can be gained will be limited by the finite-size effects, there is hope that the notable differences between the equilibrium and non-equilibrium models will not be wholly obscured.

As discussed in chapter 2, the quantity of primary concern when evaluating stochastic systems such as these is the steady state distribution $P^{*}$. From the master equation, eqn. (2.1), it is clear that $P^{*}$ must satisfy the relationship

$$
P^{*}(\tau+1)=W_{j}^{k} P^{*}(\tau)
$$

where the transition matrix $W_{j}^{k}$ has been rewritten in terms of states $j$ and $k$ and contains the transition probabilities to and from all states. It is therefore an $N \times N$ matrix, where $N$ is the total number of states accessible to the system. For the purposes of this study, these rates are considered a given part of the model and are dictated by the Metropolis algorithm. 
With $W_{j}^{k}$ known, it is simple in principle to solve for $P^{*}$ by finding the eigenvector associated with the unity eigenvalue. As already discussed, this is not generally feasible in practice. For a conserved system at $\mathcal{M}=0$, the number of states is given by $N=\left(\begin{array}{c}L \\ L / 2\end{array}\right)$. Due to this fact even a system of modest size, say $L=100$, has on the order of $10^{29}$ accessible states. At this size it is infeasible to construct $W_{j}^{k}$, let alone solve the associated eigenvalue problem. On the other hand, for $L=6$ the total number of states is only 20, which is a much more manageable number. It is for this reason that a system of this size will be explored despite the inherent limitations of the conclusions that may be drawn.

The first step in solving this problem is the construction of $W_{j}^{k}$. While the dynamics of the system are ergodic and all states are ultimately accessible from any initial state, not all states are accessible in a single time step, which is the process which eqn. (3.4) describes. It is therefore necessary to consider the adjacency of the states within the configuration space when constructing $W_{j}^{k}$. This adjacency relies solely on the dynamics of the system, which shouldn't be terribly surprising considering how the total number states relies directly on the dynamics as well. This fact will have consequences when small systems of the nonequilibrium $2 T$ model are considered, as the connections between microstates will not be changed - the dynamics are equivalent after all - but rather the rates between theses states will be modified. This fact alone must therefore account for the rich differences in behavior that will be observed.

Presently there are no systematic means by which to generate the adjacency matrix for a given dynamics. This thereby requires that the states and their relationships be determined manually. While not difficult, this can be a tedious task and thereby further limits the size of systems which are practical to analyze. It should be possible to develop an algorithm to build these relationship automatically, and such a task may be worthwhile by bringing insight into the behavior of systems in which finite-size effects are somewhat less severe.

Before enumerating the 20 states of the $L=6$ system, it is useful to introduce the bond representation of the system states. In such a representation the product of each pair of nearest neighbor spins, $\sigma_{i} \sigma_{i+1}$, is considered in place of the the spin value $\sigma_{i}$ of each lattice site. The naming of this quantity owes to the fact that it is directly proportional to the energy of the bond between particles at a given lattice point. The primary advantage of this representation is that it automatically respects the parity symmetry of the model, in which each spin is replaced by a spin of the opposite sign. In this way the number of states that need to be considered is immediately halved while the physics is unchanged. 
Table 3.1: The 10 energetic configurations for $L=6$ and $\mathcal{M}=0$. The cell colors correspond to the node colors in Fig. 3.10.

\begin{tabular}{|c|c|ccccc|c|}
\hline Number & \multicolumn{5}{|c|}{ Configuration } & Energy \\
\hline 1 & - & $\mid$ & - & - & $\mid$ & - & -2 \\
2 & $\mid$ & - & - & $\mid$ & - & - & -2 \\
3 & - & - & $\mid$ & - & - & $\mid$ & -2 \\
\hline 4 & - & $\mid$ & $\mid$ & $\mid$ & - & $\mid$ & 2 \\
5 & $\mid$ & - & $\mid$ & $\mid$ & $\mid$ & - & 2 \\
6 & - & $\mid$ & - & $\mid$ & $\mid$ & $\mid$ & 2 \\
7 & $\mid$ & $\mid$ & $\mid$ & - & $\mid$ & - & 2 \\
8 & $\mid$ & $\mid$ & - & $\mid$ & - & $\mid$ & 2 \\
9 & $\mid$ & - & $\mid$ & - & $\mid$ & $\mid$ & 2 \\
\hline 10 & $\mid$ & $\mid$ & $\mid$ & $\mid$ & $\mid$ & $\mid$ & 6 \\
\hline
\end{tabular}

A '-' will be used to represent a 'satisfied' bond between particles $\left(\sigma_{i} \sigma_{i+1}=1\right)$, while a '"' will be used for a 'broken' bond $\left(\sigma_{i} \sigma_{i+1}=-1\right)$. The 10 unique energy configurations are displayed in Table 3.1, grouped and colored by the total energy of the state. The only remaining chore is to determine which of these states are adjacent under Kawasaki dynamics. This adjacency can be effectively captured with the configuration space network, which is displayed in the left part of Fig. 3.10. At first glance, the numerical ordering of these states may seem both arbitrary and counterintuitive. The true motivation for this will become clear in Section 4.4 when the $2 T$ model is considered.

With the adjacency matrix in hand, it is now a simple task to construct $W_{j}^{k}$ and solve for $P^{*}$. The right part of Fig. 3.10 shows the resultant Boltzmann distribution over the 10 states of the system for $T=5$. As expected, the probability of a given state is only a function of the energy of the state and the lowest energy states are the most likely to occur. As the temperature is lowered the distribution begins to be dominated by the three ground states, and therefore the average properties of the system are heavily influenced by these specific configurations. While this provides a nice confirmation that the approach to the solution is correct, there is not much else of interest with these results.

The standard homogeneous case does, however, provide a nice foundation for viewing the 2J model in the same manner. As has been emphasized numerous times, changing the coupling strength over a region of the model does nothing to change the accessible configurations or the adjacency matrix. In this sense, the topology of the configuration network is unchanged. What does change, however, are the energies of the configurations 

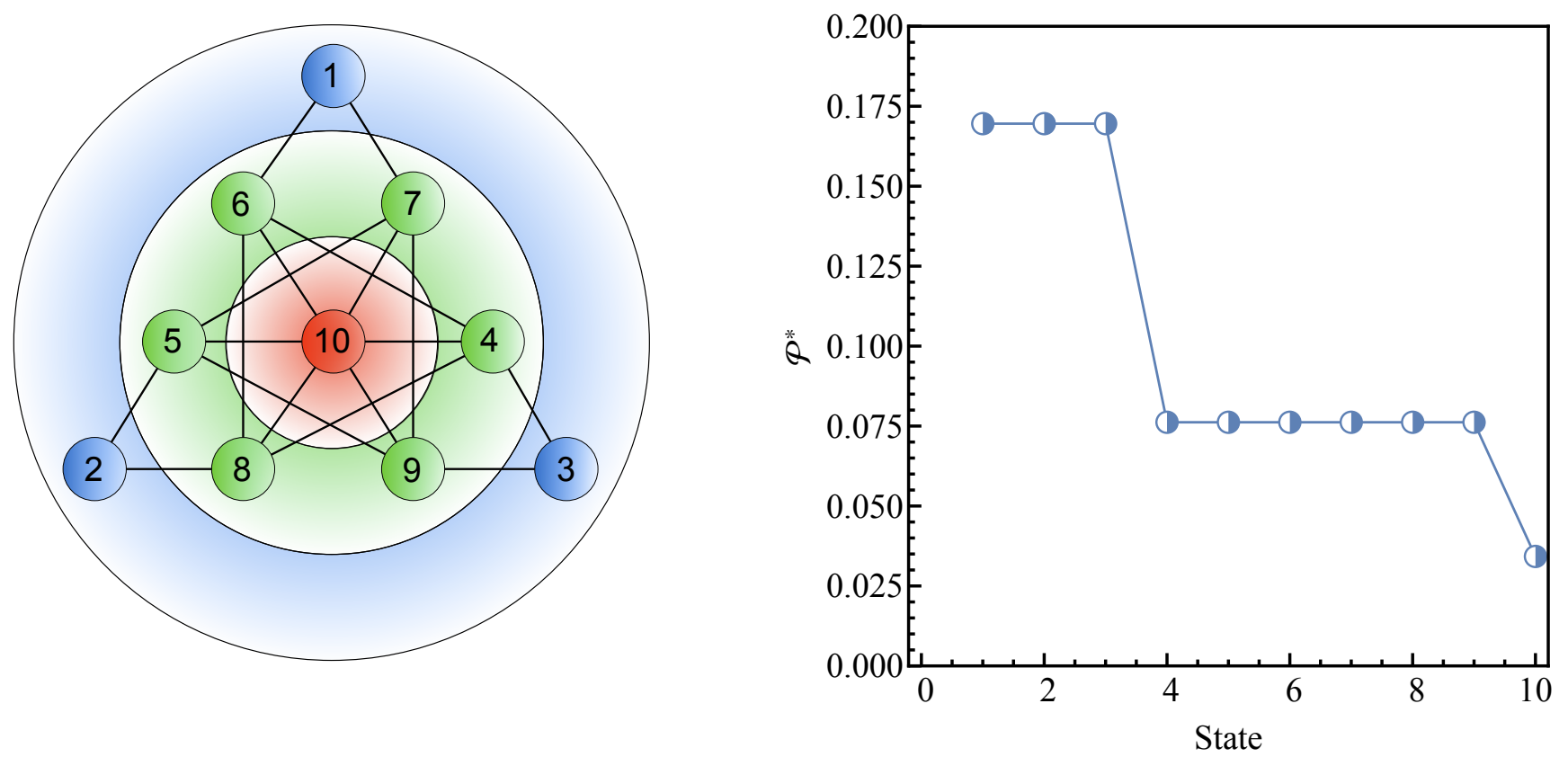

Figure 3.10: The left image displays the configuration network for Kawasaki dynamics acting on $L=6, \mathcal{M}=0$. The numbers correspond to the states indicated in Table 3.1, while the colors refer to the corresponding energy level. In the right plot, the calculated values of $P^{*}$ for this system at $T=5$ are indicated.

$$
\mathcal{H}=-\sum_{i=1}^{L} J_{i} \sigma_{i} \sigma_{i+1}
$$

The introduction of the $J=0$ window over the center of the model sets the energy of the associated bonds to zero. Table 3.1 can be updated to reflect this change by replacing the 'broken' or 'satisfied' bonds from the window region with a ' 0 '. This change, along with the new state energies, is shown in Table 3.2 for a $2 J$ model with a window containing two bonds.

The $2 J$ window introduces two noteworthy changes to the energetics of the $L=6$ model. The first is the creation of a fourth energy level immediately above the groundstate. The separation between adjacent energy levels is halved in comparison with the standard homogeneous model, and a number of states are shifted up or down in energy. As the configuration network in Fig. 3.11 shows, this does not change the topology of the network, and the changes in energy still allow detailed balance to be satisfied. Of course, the specific probabilities of the states $P^{*}$ will be changed with respect to the new energies, but the Boltzmann distribution still applies. This can be seen in the distribution shown in the right in Fig. 3.11. The numbering of the states has been kept consistent with those of Table 3.1, but if they were grouped based on energy it would be even more apparent that the distribution is a function 
Table 3.2: The 10 energetic configurations for $L=6$ and $\mathcal{M}=0$ in a $2 J$ model with $w=2$. The cell colors correspond to the node colors in Fig. 3.11.

\begin{tabular}{|c|cccccc|c|}
\hline Number & \multicolumn{5}{|c|}{ Configuration } & Energy \\
\hline 1 & - & $\mid$ & 0 & 0 & $\mid$ & - & 0 \\
\hline 2 & $\mid$ & - & 0 & 0 & - & - & -2 \\
3 & - & - & 0 & 0 & - & $\mid$ & -2 \\
\hline 4 & - & $\mid$ & 0 & 0 & - & $\mid$ & 0 \\
5 & $\mid$ & - & 0 & 0 & $\mid$ & - & 0 \\
\hline 6 & - & $\mid$ & 0 & 0 & $\mid$ & $\mid$ & 2 \\
7 & $\mid$ & $\mid$ & 0 & 0 & $\mid$ & - & 2 \\
8 & $\mid$ & $\mid$ & 0 & 0 & - & $\mid$ & 2 \\
9 & $\mid$ & - & 0 & 0 & $\mid$ & $\mid$ & 2 \\
\hline 10 & $\mid$ & $\mid$ & 0 & 0 & $\mid$ & $\mid$ & 4 \\
\hline
\end{tabular}

of the four distinct energy levels only.

The most important thing to note from Fig. 3.11 is how the number of ground states is reduced to two by state 3 being shifted to the next higher energy level. This has important consequences for the low temperature behavior of the model. As noted above, at low temperatures $P^{*}$ is completely dominated by the ground states in a small model such as this. By state 3 being shifted up in energy, only states 1 and 2 are crucial at very low temperatures. This is notable by the fact that state 3 was the one ground state which contained only satisfied bonds within the window, and in this sense had an ordered window. By shifting this state out of the ground state this ensures that the window is more likely to be disordered even at lower temperatures, which is precisely what is seen in the results of the $2 J$ model for larger system sizes (see Figs. 3.8 and 3.9). For this small model, there are some hints of the behavior of the larger scale systems already present. Whether this remains the case for the $2 T$ model as well will be discussed towards the end of the following chapter. 

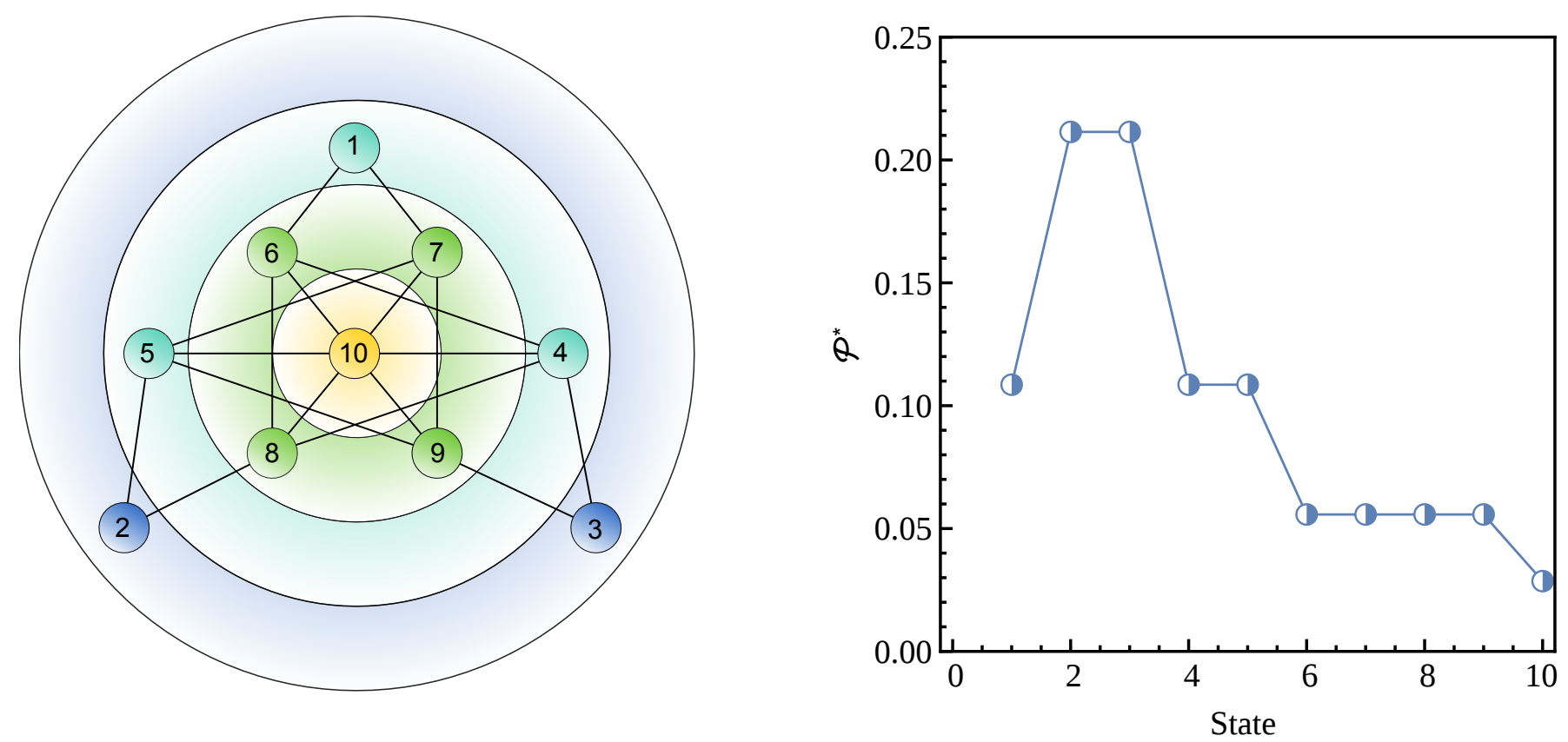

Figure 3.11: The left image displays the configuration network for Kawasaki dynamics acting on the $2 J$ system with $L=6, \mathcal{M}=0$, and $w=2$. The numbers correspond to the states indicated in table 3.2, while the colors refer to the corresponding energy level. In the right plot the calculated values of $P^{*}$ for this system at $T=3$ are indicated. 


\section{Chapter 4}

\section{The two-temperature non-equilibrium model}

We now proceed to discuss the two-temperature non-equilibrium ring and the intriguing and unexpected features that emerge from a local breaking of detailed balance. The investigation of steady state and relaxation properties is mainly done through the same quantities as those introduced in the previous Section for the characterization of the standard Ising and $2 J$ models.

\subsection{Steady state space-time plots}

A good starting point for appreciating the nature of the non-equilibrium steady states that arise in the two-temperature ring are the space-time plots of systems that have settled into the steady state.

The space-time plot shown in the left panel of Fig. 4.1, with the temperature outside the window being at $T=1$, is in stark contrast to the space-time plots in Figs. 3.4 and 3.8 for the standard Ising system and the equilibrium $2 J$ system at the same temperature. While phase-separated domains still form as in the equilibrium model, the presence of one large domain which envelopes the central window (indicated by red lines) is noteworthy. This domain remains pinned to the location of the infinite temperature window. This behavior is particularly strange when considered in light of the equilibrium system at infinite temperature where the spins are distributed in a completely uncorrelated manner. It is thus surprising that the infinite temperature window of the two-temperature model is consistently the most ordered region of the lattice!

The space-time plots provide strong indications of a crossover between different steady state regimes. This transition can be observed when varying either the size of the infinite tem- 

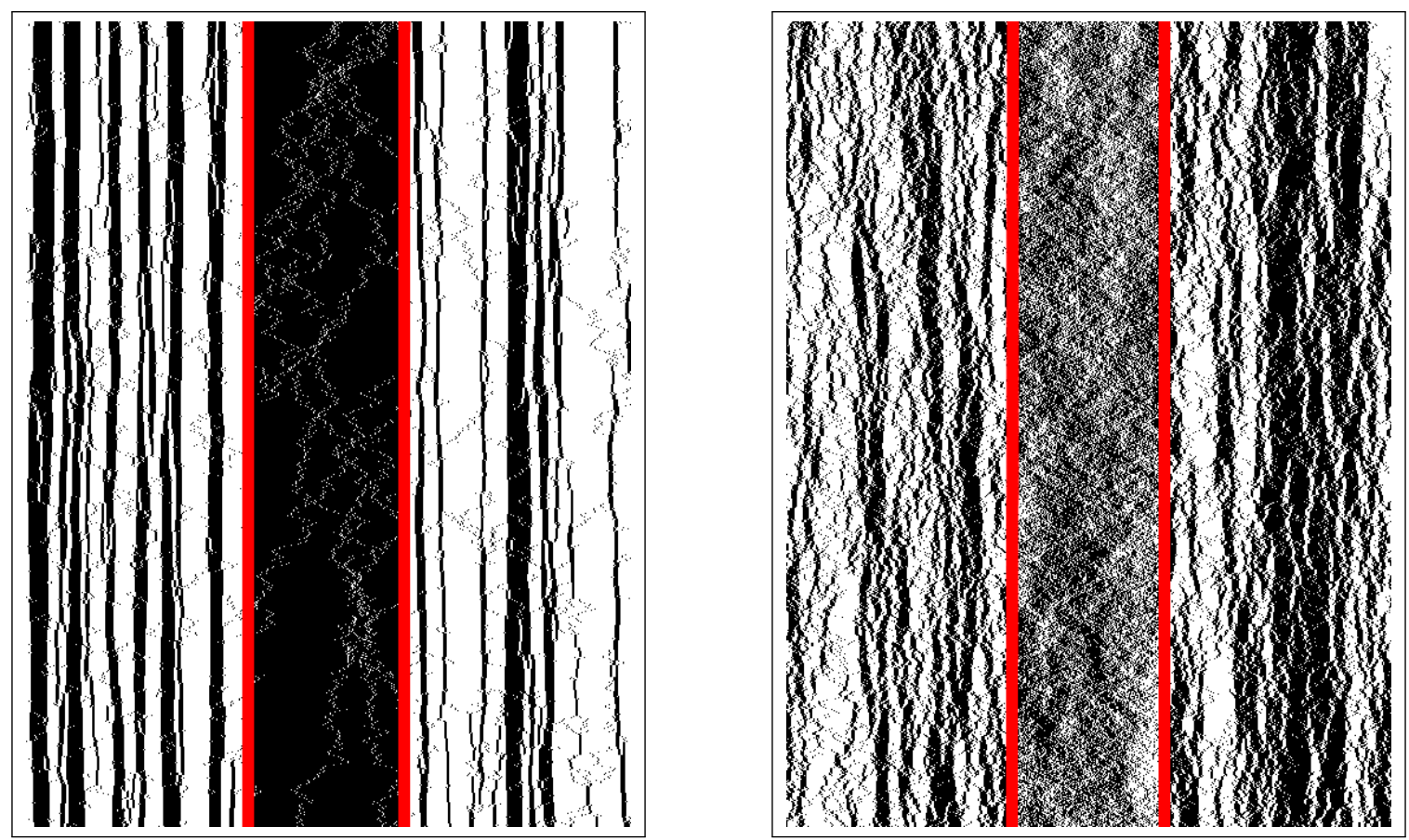

Figure 4.1: Space-time plots of the two-temperature model at different values of $T$. The plots are from a system of length $L=512$, with a window of size $w=128$ wherein the system is in contact with a reservoir at temperature $T_{w}=\infty$. For the left system we have $T=1$, whereas for the right system $T=1.75$. The boundaries between the regions are highlighted in red. Both plots show the evolution of the system over a total of $2^{12}$ MCS after an initial relaxation period of $2^{30}$ MCS, where two consecutive vertical lines are separated by 6 MCS.

perature window or the temperature of the lattice sites outside the window, T. Fig. 4.1 demonstrates the effect due to varying $T$. At larger values of $T$ (illustrated in the right panel in Fig. 4.1), the infinite temperature window behaves more or less as expected by being more disordered than the much colder, surrounding lattice. When $T$ is lowered, a crossover takes place to a state where the region within the window is much more ordered, despite being coupled to a much higher temperature bath than that in the surroundings (illustrated in the left panel of Fig. 4.1).

The left panel of Fig. 4.2 demonstrates how the changing of the window size $w$ can influence the presence of the ordered state. In this space-time plot the window is clearly in a disordered state. This is despite the fact that $T$ is sufficiently low to form the ordered state with a smaller $w$, as is the case in the left panel of Fig. 4.1. This shows that the crossover is sensitive to both the values of $T$ and $w$, and any critical point that exists will rely on some non-trivial relationship between the two.

The logarithmic space-time plot in the right panel of Fig. 4.2, while physically identical to 
the left panel of Fig. 4.1, reveals new features of the system by exploring the ordered state over much longer time scales. Of particular note in this image are the alternating horizontal bands of particles and holes which appear within the window at very long times. These are indicative of tunneling events, in which the system transitions from an ordered window filled with particles to one filled with holes and vice versa. It is not surprising that the system should end up finding itself in either of these ordered states, as the parity between the spins guarantees that these configurations should be equally likely. Nonetheless, it is interesting to see these very stable ordered states dissolve and reform when viewing the dynamics of the system over very long times. The window is actually in a very disordered state during the tunneling event, but the duration of these events appears to be many orders of magnitude shorter than the ordered phase itself and therefore contribute little to the average behavior of the window. There are indications, however, that these tunneling events grow in duration as the overall system size is increased, and that they are eventually comparable (and longer!) than the ordered phases. This represents a form of phase coexistence within the model, and will be explored in more detail in the following sections.

There is another perspective to this remarkable crossover, namely, when $T_{w}$ is raised from $T$ (for the case $T=1$, say) to $T_{w}=\infty$. Then, within the window and contrary to expectations, order will gradually emerge from disorder! Such surprising phenomena have been reported decades ago in the context of the two-temperature Ising lattice gas in two dimensions [27, 37-41]. Finding this unexpected feature - the increase of order despite an apparent increase of fluctuations - in driven continuum systems, Helbing, et. al. coined it 'freezing by heating' [52]. In a more general setting, we may regard these counter-intuitive behavior as 'negative response,' a property that can be expected in a wide class of non-equilibrium statistical systems [53].

\subsection{Relaxation process}

Before exploring and quantifying the steady state features further, we need to consider the issue of how the system relaxes towards the steady state. The log-binning procedure will be implemented to probe the relaxation process in the same vein as the equilibrium model was explored in Section 3.1. As shown in the following, in addition to providing a means to confirm entrance into the steady state, these measurements contain important hints to the process which causes the window to display this high level of order.

The addition of an infinite temperature window within the lattice dramatically changes the manner in which the system relaxes for smaller values of $T$, as becomes obvious when comparing the time-dependent energy in Fig. 4.3 with that in Figs. 3.1 and 3.2. Whereas Fig. 4.3a contains several curves with different window sizes for a system with $L=512$ sites at $T=1$, Fig. 4.3b shows the relaxation of the energy when varying $L$ and $w$ in such a way that the ratio $R=w / L$ is kept constant. 

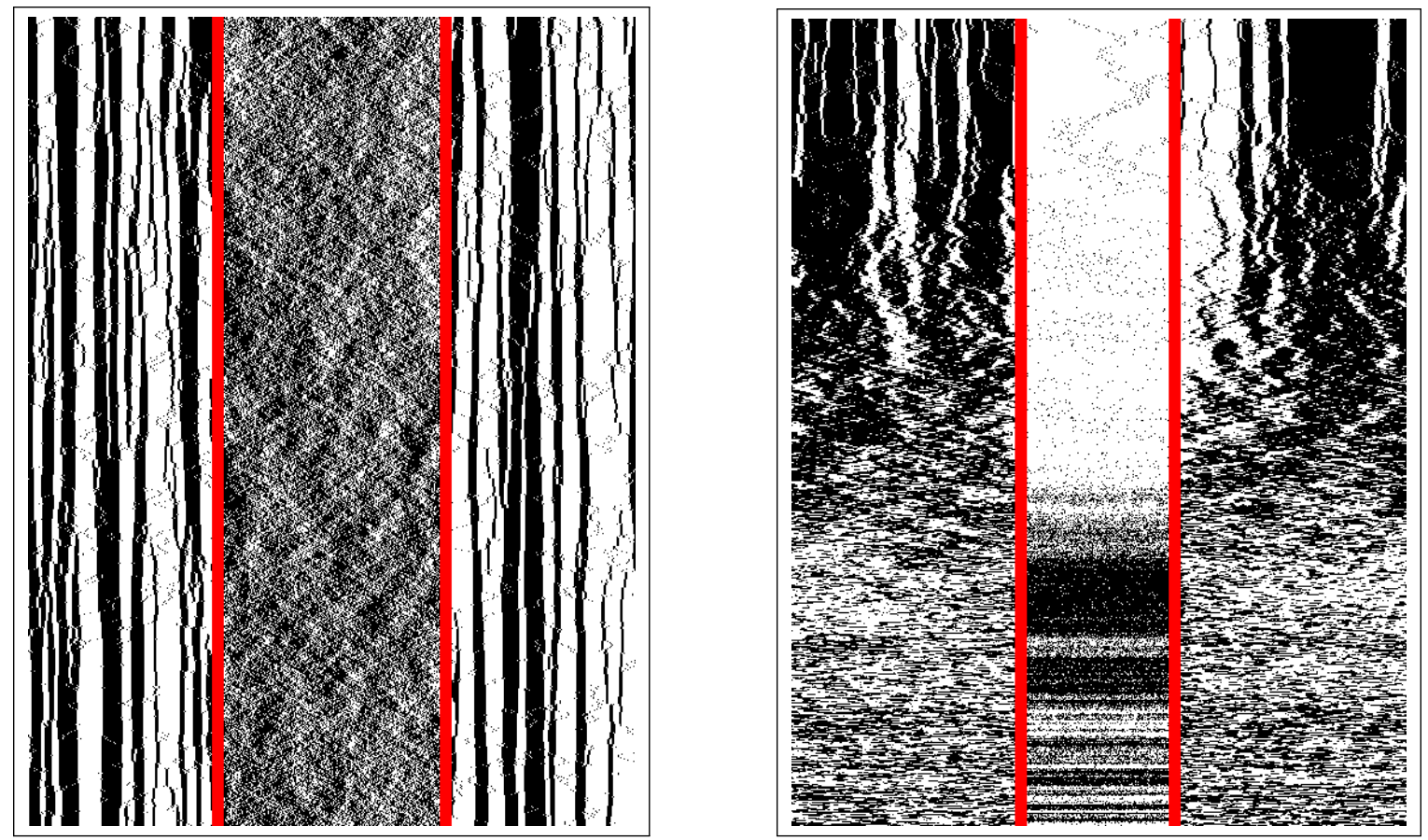

Figure 4.2: Space-time plots of the two-temperature model at different values of $w$ and over different lengths of time. The left plot is a $2 T$ system with $L=512$, $w=192$, and $T=1$. For a window of this size, the ordered phase does not form even for this relatively low value of $T$. This plot shows the evolution of the system over a total of $2^{12}$ MCS after an initial relaxation period of $2^{30}$ MCS, where two consecutive vertical lines are separated by $6 \mathrm{MCS}$. The right plot shows a logarithmic space time for the system with $w=128$. Here the relaxation period is again $2^{30} \mathrm{MCS}$, but a total of $2^{25} \mathrm{MCS}$ of evolution are shown in the plot. The boundaries between the regions are highlighted in red.

There are a couple of particularly interesting features which distinguish the two-temperature curves from the equilibrium ones. First, the non-equilibrium curves are characterized by the existence of a metastable state followed by a sudden increase in fluctuations and decline in average energy. This metastable state is revealed by the flattening of the curve and the reduction in statistical error which mimics the behavior of a system entering a steady state. The duration of this metastable state is proportional to the size of the window, specifically scaling with $(w / 2)^{3}$. This is reminiscent of the scaling seen within a one-dimensional Ising system with conserved dynamics where the domain size scales as $t^{1 / 3}$ [51], indicating that domain growth outside the window drives this phenomenon. Using the above scaling relationship, a domain of size $w / 2=64$ should take approximately $2^{18}$ MCS to form. This time, indicated by the dashed vertical line in Fig. 4.3a, roughly corresponds with the end of the metastable state for the curve with $w=128$. 

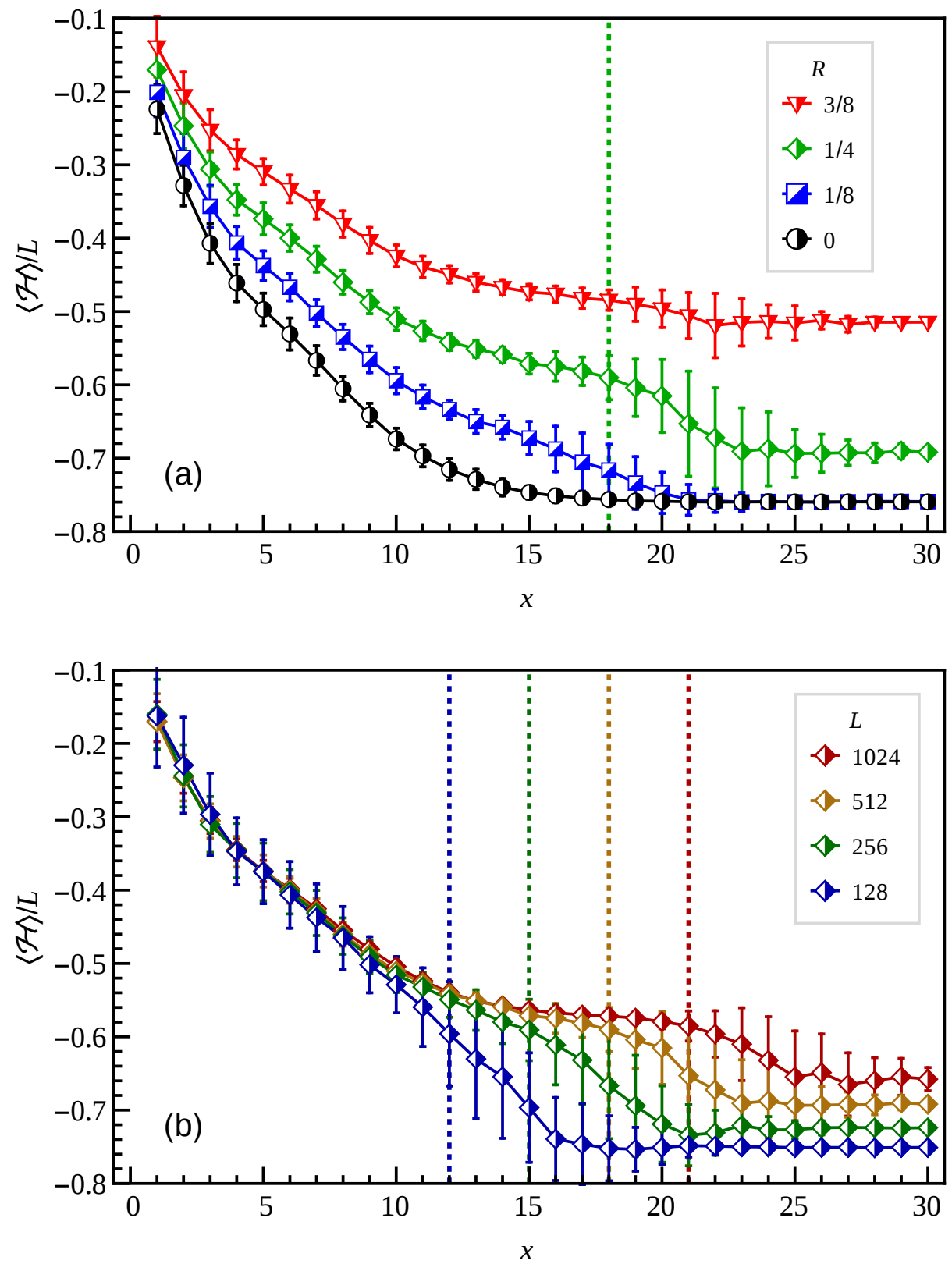

Figure 4.3: (a) Log-binning relaxation of the average energy density for $T=1$ with fixed system size $L=512$ and varying window size $w$ (yielding different values for the ratio $R=w / L$, see the figure legend). The dashed vertical line is placed at the point at which the green curve with $w=128$ exits the metastable state, which is equivalent to $(w / 2)^{3}=64^{3}=2^{18}$. (b) Log-binning relaxation of the average energy density for fixed ratio $R=w / L=1 / 4$ and $T=1$. The dashed vertical lines are placed to correspond with $(w / 2)^{3}$ for their respective curves (left most curve located at $16^{3}$, right most curve located at $128^{3}$ ). Reprinted figure with permission from N.Borchers, M. Pleimling, and R.K.P. Zia, Physical Review E, 90(6):062113, 2014. Copyright 2014 by the American Physical Society. 
This idea is further supported by Fig. 4.3b, which displays the average energy density for various system sizes $L$. Here the ratio of the window to lattice size, $R=w / L$, is kept fixed at $1 / 4$. After the initial slope on which all of the curves collapse - due to initial cluster formation - the curves then diverge as they enter the metastable state. This is to be expected if the above reasoning holds since each curve has a different associated window size, and thus a proportionately different time to exit the metastable state. Again, the dashed vertical lines indicate the time this should take for the correspondingly colored curves. As before, the match indicates that the onset of the ordered phase scales with $(w / 2)^{3}$. This points to the fact that the cluster formation outside of the window strongly influences the emergence of the ordered window.

As a final demonstration of the importance of the $t \propto w^{3}$ scaling within this system, the upper panel Fig. 4.4 mirrors Fig. 4.3b, but now with the time- and ensemble-averaged window magnetization $\left\langle\left|\bar{M}_{w}\right|\right\rangle$ as the quantity of interest. We should emphasize here that for $\left\langle\left|\bar{M}_{w}\right|\right\rangle$, we first average the window magnetization from the start to time $t$, which yields a quantity which may be either positive or negative and eventually goes to zero as enough of phase space is explored and the spin symmetry takes over. The absolute value of the time-average is then taken prior to constructing the ensemble average, here represented by the angular brackets and again being performed over 40-100 independent realizations. The construction of this quantity, while a bit unorthodox, has a key advantage in that it not only shows distinctly how long it takes for the ordered state to develop, but also how long it takes for an ordered state of opposite sign to displace the original configuration. This point can be identified as the peak within the curves, and the time for this to take place scales very well with $(2 w)^{3}$. In the lower panel we show for comparison the same quantity for the standard Ising model. By construction, $\left\langle\left|\bar{M}_{w}\right|\right\rangle$ also displays maxima for that case, but the heights of these maxima are much smaller than for the $2 T$ model. This reflects the fact, see the time trace in Fig. 3.4, that every choice of the window will include both positively and negatively magnetized domains, in contrast to the non-equilibrium case where the window at infinite temperature is occupied by a single, almost perfectly ordered domain.

Returning to Fig. 4.3a, the other point of interest is the final relaxed level of the nonequilibrium system with ratio $R=1 / 8$ (which corresponds to window size $w=64$ ). What is notable in this case is that the energy per lattice site ultimately falls to nearly the same level as the equilibrium (black) curve. Fig. 4.5 demonstrates that it is even possible to have lower than equilibrium energy with the right mix of parameters. Notice that the curve for $R=1 / 8$ (which here corresponds to $w=16$ as $L=128$ ) not only approaches the value for the equilibrium model, but actually goes below it in the steady state. This is admittedly achieved for a small system size in which finite-size effects are almost certain to play a significant role. Such a result is again reminiscent of the negative response considered in $[53]$.

While the features of the relaxation process discussed above do much to reveal details about the formation and nature of the unique steady state of the $2 T$ model, from a practical 


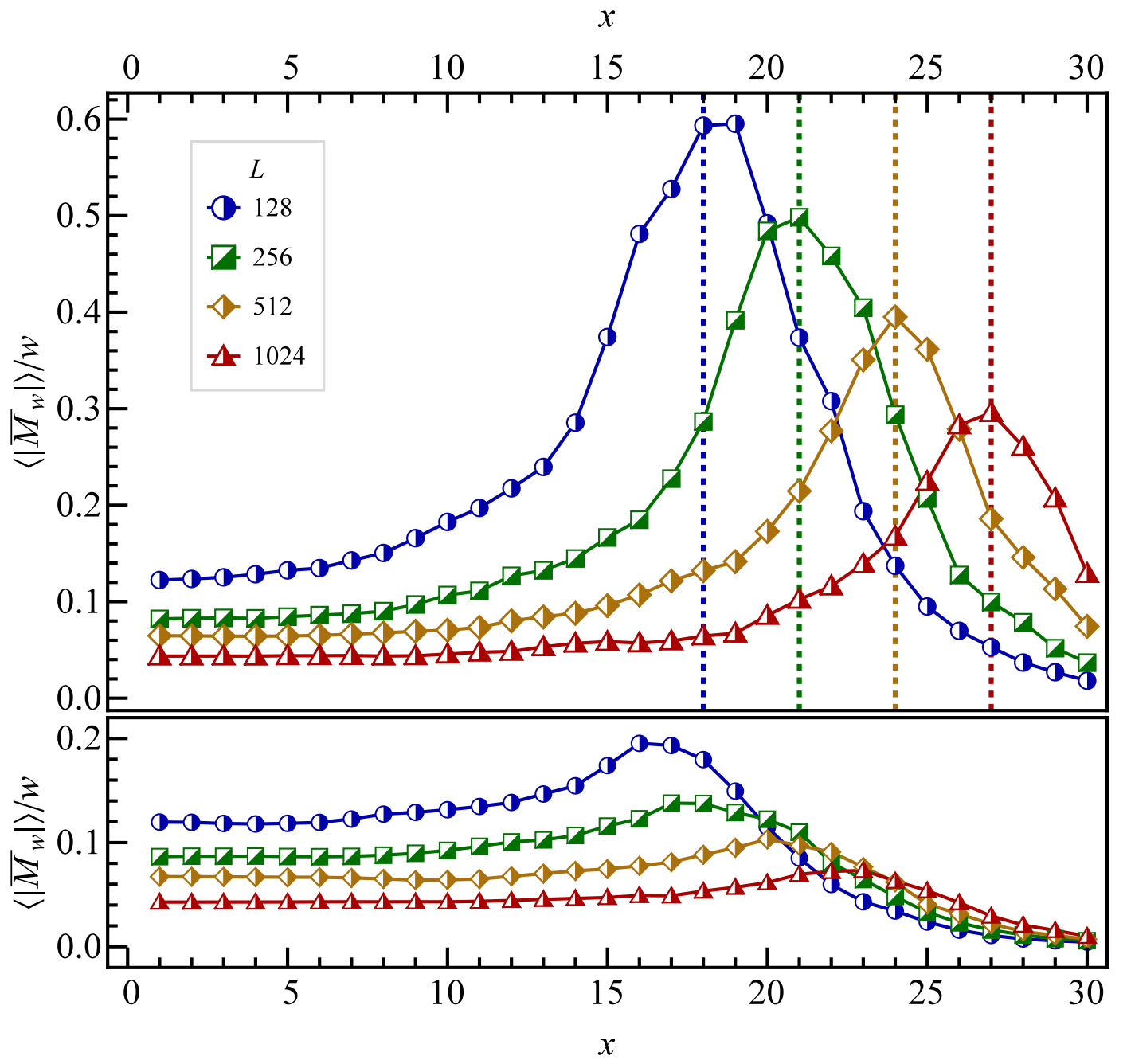

Figure 4.4: Upper panel: relaxation of the normalized average window magnetization for $R=1 / 4$ and $T=1$, see main text. Here the dashed vertical lines correspond with $(2 w)^{3}$. Lower panel: the same for the standard Ising model. Reprinted figure with permission from N.Borchers, M. Pleimling, and R.K.P. Zia, Physical Review E, 90(6):062113, 2014. Copyright 2014 by the American Physical Society. 


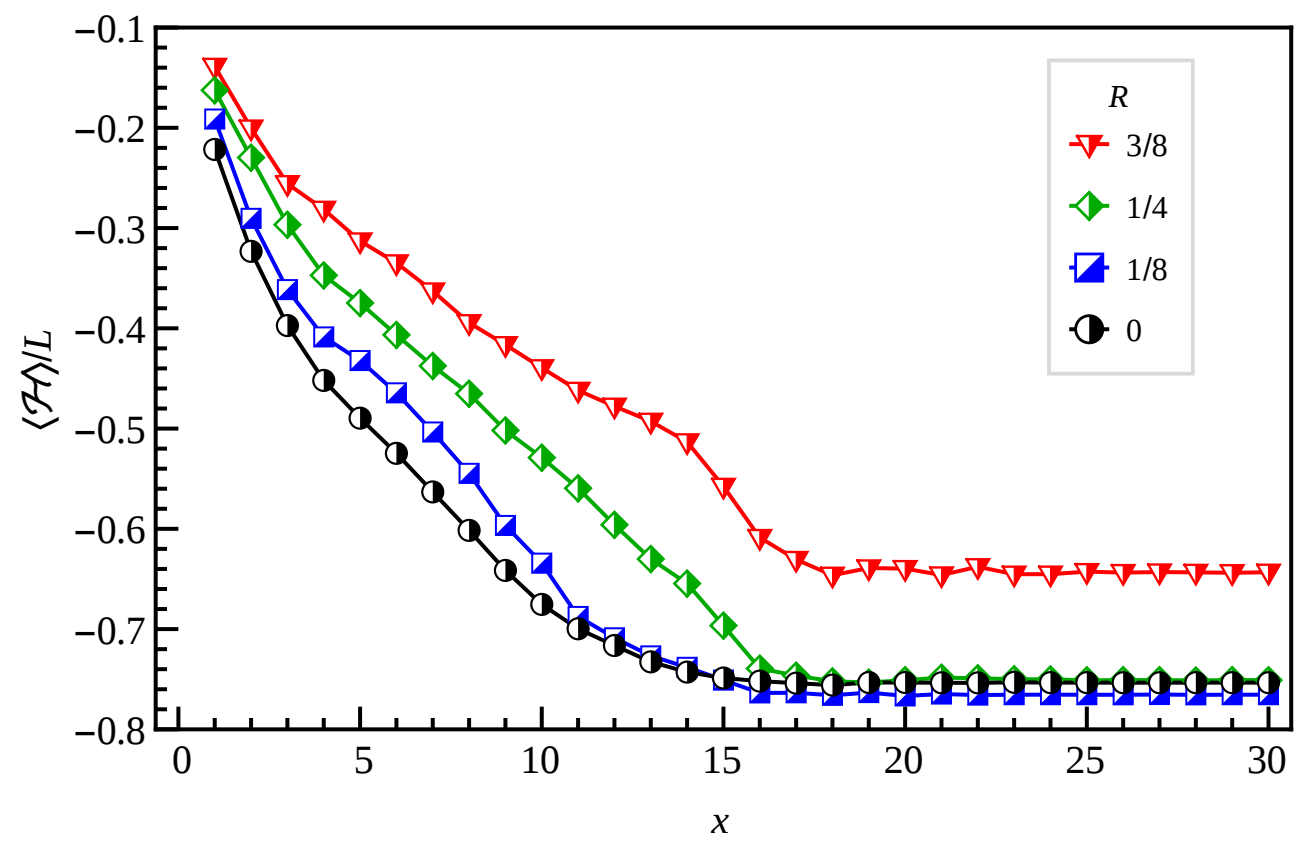

Figure 4.5: Log-binning relaxation of the average energy density for $L=128$, $T=1$, and various values of the ratio $R=w / L$. Error bars are comparable to the symbol size. Reprinted figure with permission from N.Borchers, M. Pleimling, and R.K.P. Zia, Physical Review E, 90(6):062113, 2014. Copyright 2014 by the American Physical Society.

perspective the primary utility of the log binning curves remains in confirming entrance into the said steady state. From this perspective, the log binning curves thus far displayed are less than encouraging. The presence of the metastable state in the relaxation process postpones entrance into the steady state, thereby increasing the computational time required for relaxation. This increase can be significant, particularly when considering the relaxation from a random initial condition. Comparing the lowest temperature (black) curve in Figures 3.3 and 4.6, it can be seen that the convergence between the curves happens a factor of 2 to $2^{5}$ later in the $2 T$ system. The situation is even more dire when considering the time it takes for the random initial condition curve to flatten, which may take as much as a $2^{7}$ times longer. This leads to prohibitively long relaxation times in systems with larger values of $L$, and therefore limits the ability to study such systems and explore finite-size scaling effects.

The entire relaxation process is necessitated by the fact that the random initial configurations, which are typically used to provide an unbiased starting point for the Monte-Carlo simulation, are far from representative of configurations found in the steady state. If a representative steady state configuration could be used as the initial condition for the simulation 


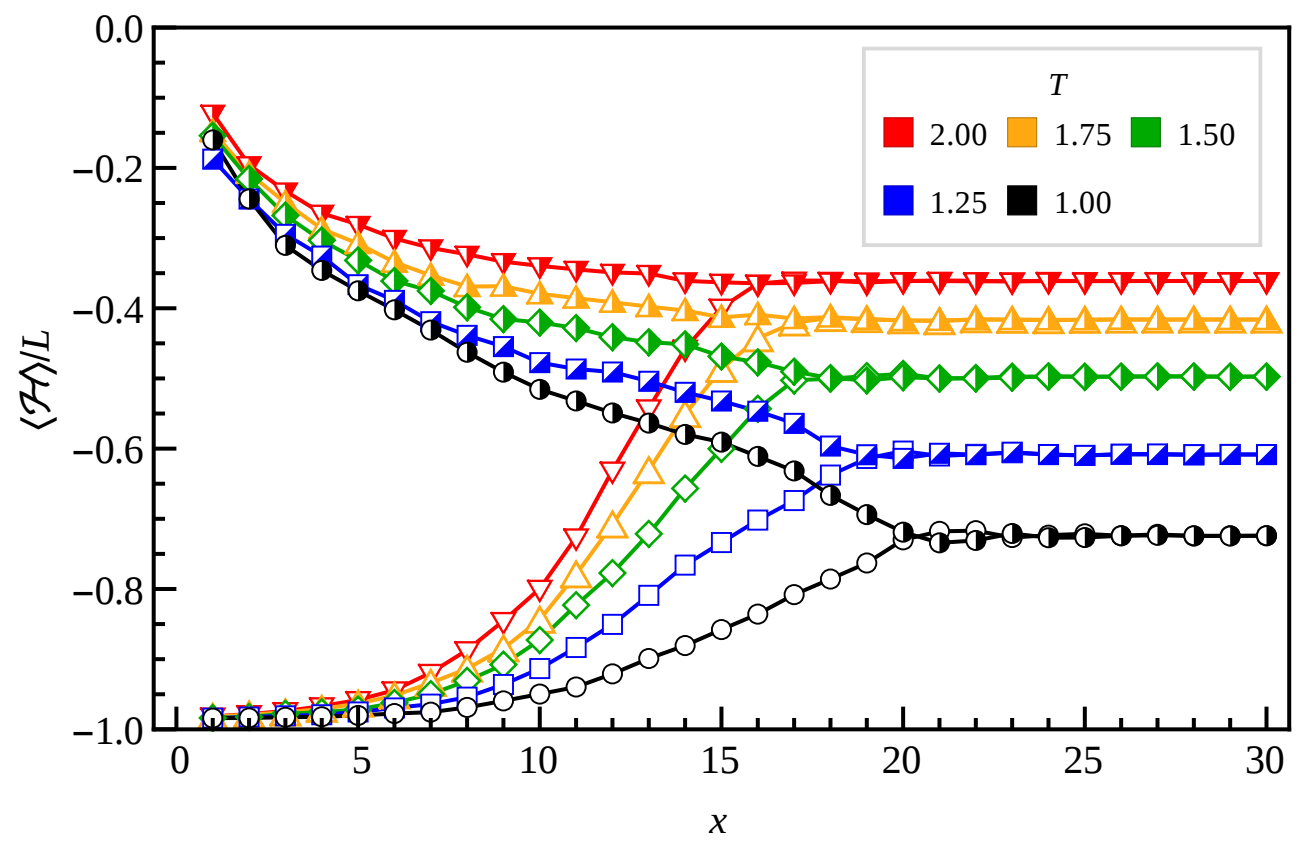

Figure 4.6: Convergence of the energy density log-binning for the $2 T$ Ising model with $L=256$ and $w=64$. The data results from averaging over an ensemble of 50 independent realizations. Filled symbols are for random initial conditions, while open symbols are for ordered initial conditions. Error bars are comparable to the sizes of the symbols.

the entire relaxation procedure could be avoided, which would allow more computation time to be spent exploring the steady state properties of the system. A straightforward solution to this issue is to simply save the final state of the system from all simulations which enter the steady state. These can then be used to form a repository of configurations from which to draw initial configurations for future simulations. This application of this technique to the standard equilibrium system is demonstrated by the black curve in Figure 4.7. As expected, the use of the steady state initial conditions essentially removes the need for the relaxation step in the simulation.

While such an approach is useful for exploring the steady state features of larger systems of the standard equilibrium model, it is truly essential for the $2 T$ model. Therefore the real interest of this approach lies in its application to the $2 T$ model, which is demonstrated by the black curve in Figure 4.8. While analogous to Figure 4.7, it is clear that the non-equilibrium steady state has introduced complications. While the steady state initial configurations cause the black curve to start much nearer to the actual value of the steady state energy density, the curve is not nearly as stable and flat as the respective curve in the equilibrium 


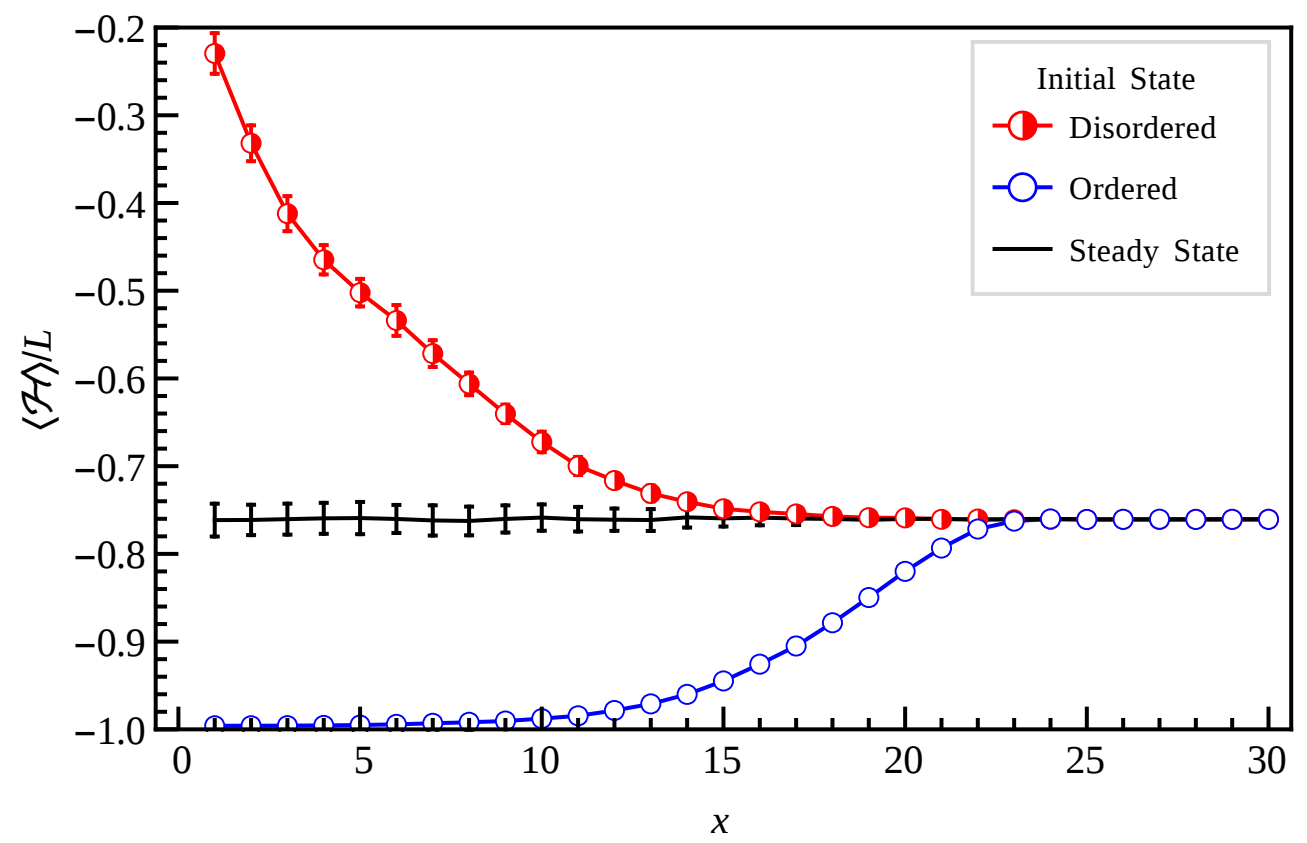

Figure 4.7: $\log$ binning of the energy density for the standard equilibrium model with $L=1024$ and $T=1$. The different curves result from using different initial conditions for the ensemble of 50 independent realizations.

plot. Such an instability in this value is an indication that the steady state has not yet been entered. Even worse is the inexplicable increase in the standard error of the curve, which reveals that ensemble of simulations used to construct this curve are anything but uniform. This is all the more puzzling since the error bars in the simulations from which the initial configurations were sampled are considerably reduced in magnitude. This is yet another signal that the steady state has yet to be established at early times of the simulations.

The obvious explanation for this behavior is that the supposed steady state initial conditions used to generate this curve are not actually representative of the steady state after all. These configurations were sampled after $2^{30}$ MCS when the system had, by all indications, entered the steady state, but it is possible that for such a large system even this length of time was insufficient to enter the steady state. Unfortunately, there is a critical issue with this interpretation. If this was simply an issue of requiring more time to enter the steady state one would expect that the errors on the steady state curve would decline much sooner than those in the other curves in Figures 4.8 since the states used for initial conditions for the steady state curve were effectively sampled from the end of the ordered and disordered curves. Rather, the errors diminish in unison with the other curves, suggesting that the same amount of time is required to enter the steady state regardless of which initial condition is chosen. 


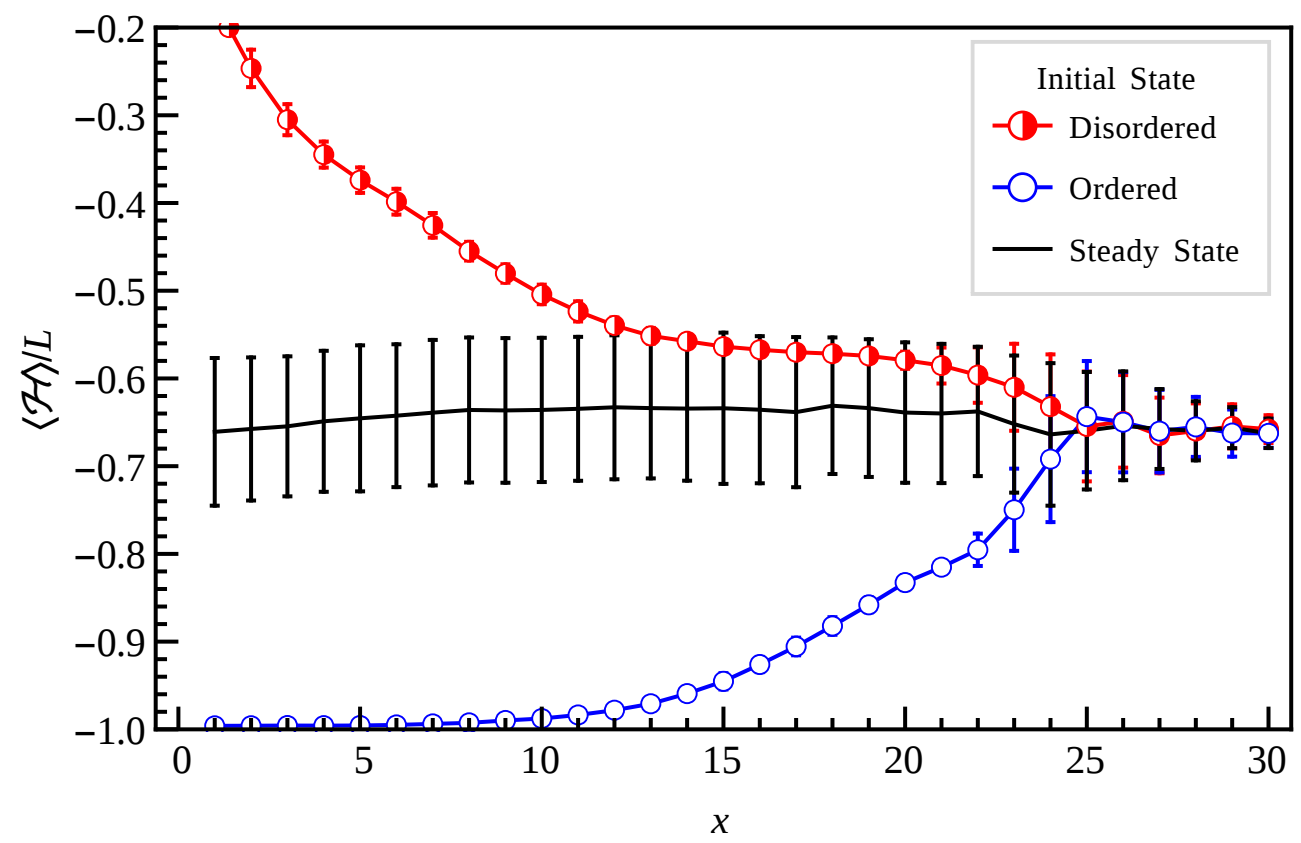

Figure 4.8: Log binning of the energy density for the $2 T$ with $L=1024, w=256$, and $T=1$. The different curves result from using different initial conditions for the ensemble of 50 independent realizations.

This explanation seems implausible at first glance, and thus an alternative line of reasoning is desired.

The true nature of the amplified error bars is revealed when viewing a sampling of the individual simulations used to construct the ensemble average which gives rise to the steady state curve in Figure 4.8. Six such of these unaveraged runs are displayed in Figure 4.9. From these runs it is clear that rather than a single steady state, the system appears to have two such states each with a distinct average value of energy density. Each state seems to be equally stable, with a lifetime of around $2^{19}$ to $2^{23}$ MCS. These states also appear equally likely based on the admittedly small sample presented in Figure 4.9. A more thorough investigation of this fact will be made in further sections. Still, from this data it is apparent that the convergence seen in 4.8 and other log binning curves for the $2 T$ model is the result of the underlying simulations being given enough time to sample both of these steady states in an individual realization. This also gives a compelling explanation for the presence of the 'meta-stable' state as the system enters one of the steady states initially, but requires further relaxation to then move to the other state. Since there are a variety of times at which this state transition can occur, the associated errors in the average would necessarily increase before settling at a new average including the energy density of both states. 
The exact nature of each of these steady states will be considered later when discussing the steady state properties of the system, but a distinction should be made at this point between the transition between these states and the tunneling events discussed in regards to Figure 4.2 in Section 4.1. Here the tunneling events specifically referred to a transition between ordered states within the window differentiated only by the sign of the total window magnetization $M_{w}$. These two possible configurations cannot represent the two steady states discovered above as they would have identical energy densities. Therefore other candidate states must be considered.

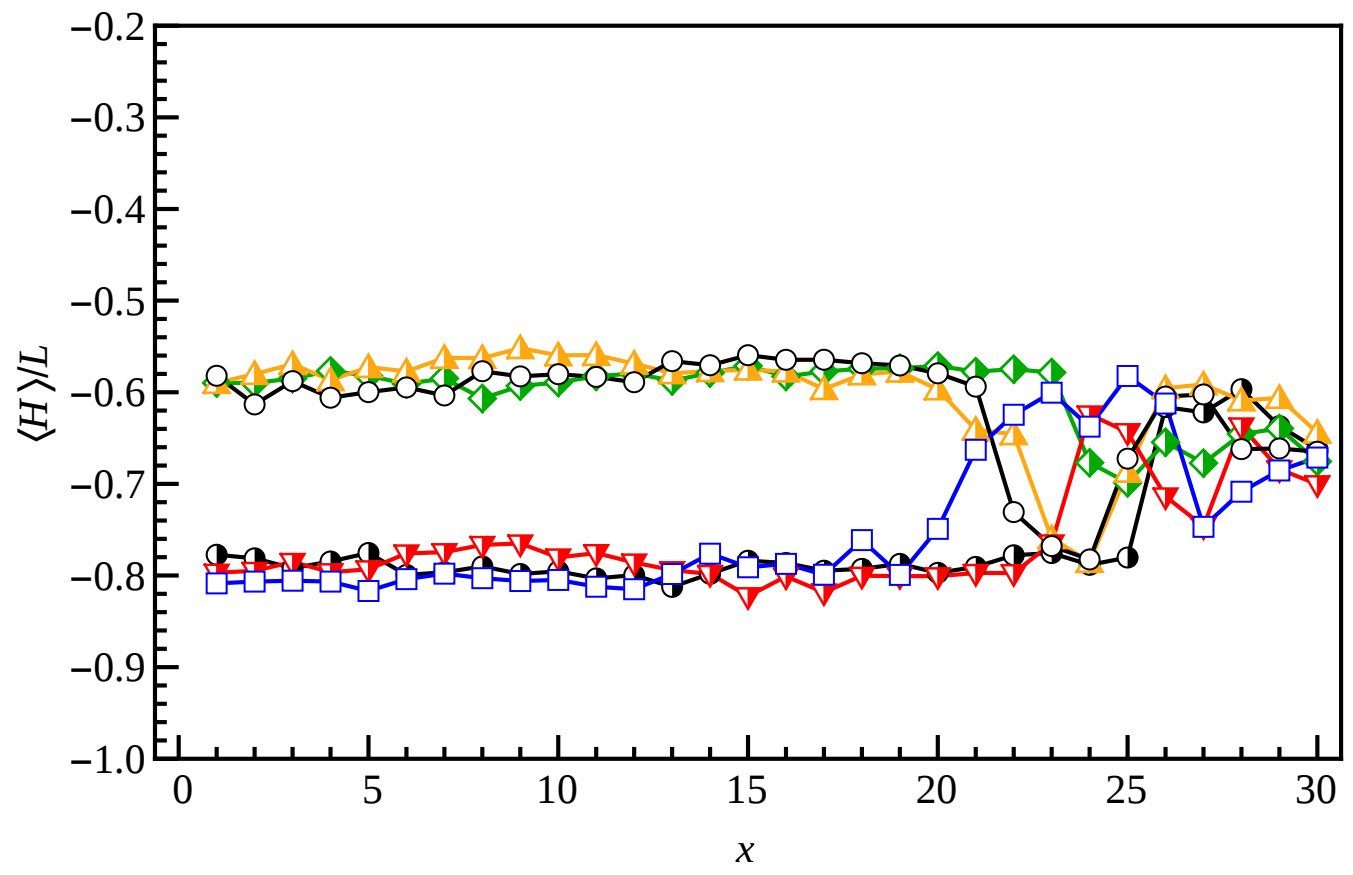

Figure 4.9: A sample of six simulations from the fifty used to construct the steady state curve in 4.8 .

\subsection{Steady state properties}

One of the best ways to statistically establish the nature of the steady state is to examine the two-spin correlations $G(i ; r)$, see Eq. (2.5). Based on the results from the two-temperature model presented thus far, it should be unsurprising that the corresponding correlation differs dramatically from that found in the standard equilibrium model, see Fig. 3.6. Since the translational invariance inherent in the equilibrium model is broken by the inclusion of the second temperature domain, it becomes necessary to specify the reference lattice site $i$. In 
the case of Fig. 4.10 the center of the window, located at $i=(w+1) / 2$, is chosen as this reference point.

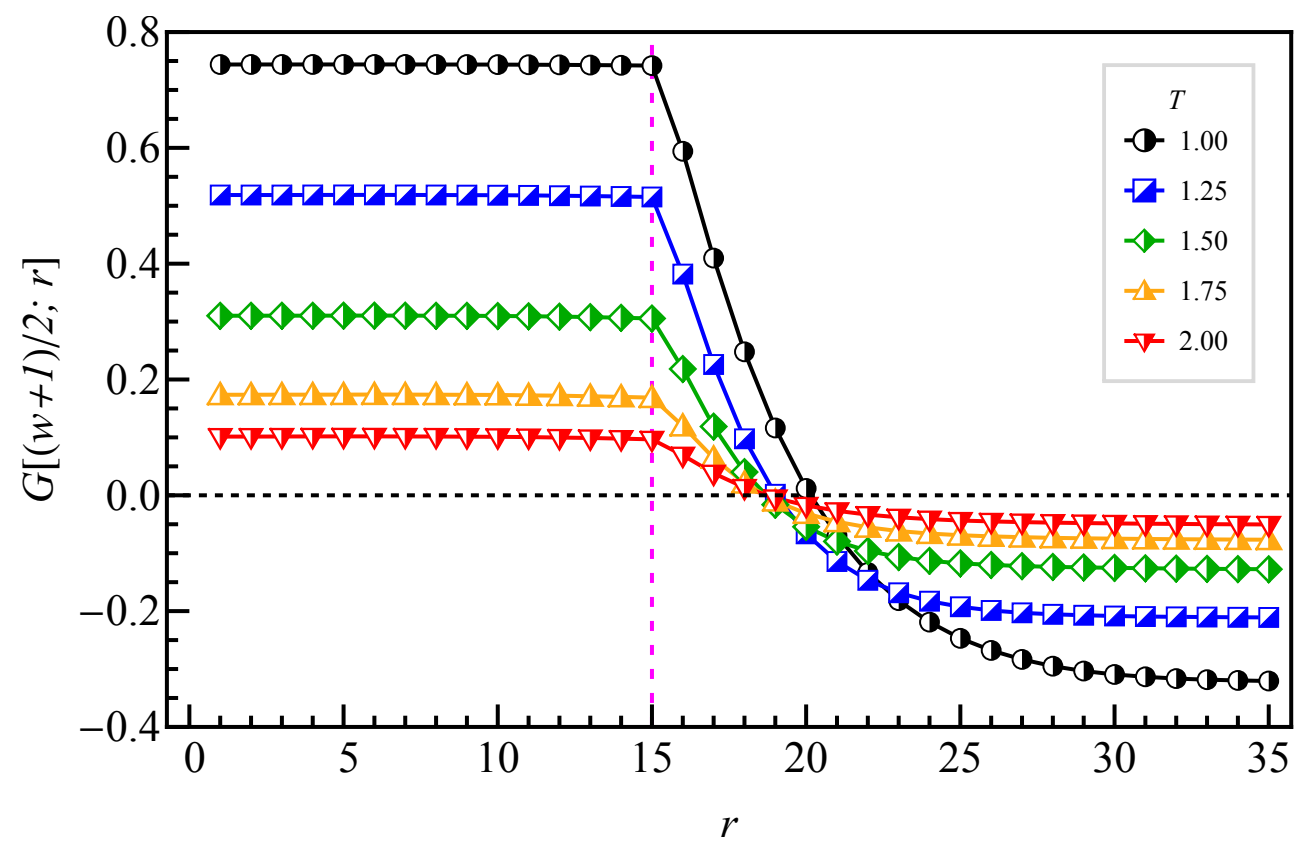

Figure 4.10: $G((w+1) / 2, r)$ for the two-temperature Ising ring with $L=127$, $w=31$, and various values of $T$. Here the lattice site $i=(w+1) / 2$ is the site in the middle of the window of width $w$. The end of the window is indicated by the dashed vertical line. Reprinted figure with permission from N.Borchers, M. Pleimling, and R.K.P. Zia, Physical Review E, 90(6):062113, 2014. Copyright 2014 by the American Physical Society.

The first feature of note is that $G((w+1) / 2 ; r)$ is almost perfectly constant over the entirety of the window. The value of this plateau agrees very well with $\left\langle M_{w}^{2}\right\rangle / w^{2}$ and decreases for increasing $T$, thereby serving as an indicator of the frequency of oppositely aligned spins entering the window. Fig. 4.10 also shows that in the region immediately outside of the window the correlation decays exponentially and roughly fits the exponential behavior of (3.3) with different constraints.

Further information about the nature of the steady state can be obtained from the average energy of the bonds between lattice sites $u_{i}$, see Eq. (2.8). Displayed in Fig. 4.11, this quantity reveals the pronounced effect the boundary between the two regions has. The equilibrium energy of the standard Ising model, indicated by the horizontal magenta line at $\approx 0.76$, is approached by the two-temperature curve far from the window, which indicates that the two-temperature model behaves more or less as the equilibrium system deep within 
the primary lattice. As the boundary between the two temperature regions is approached, however, the true influence of the point of broken detailed balance is displayed. There is a sharp, discontinuous jump at the boundary between the regions, with the average bond energy much higher immediately outside the window than inside. This indicates that the lattice sites immediately outside the window are notably more disordered than those immediately inside the boundary. This is true even for larger window sizes in which the energy inside the window is significantly higher than the equilibrium bond energy.

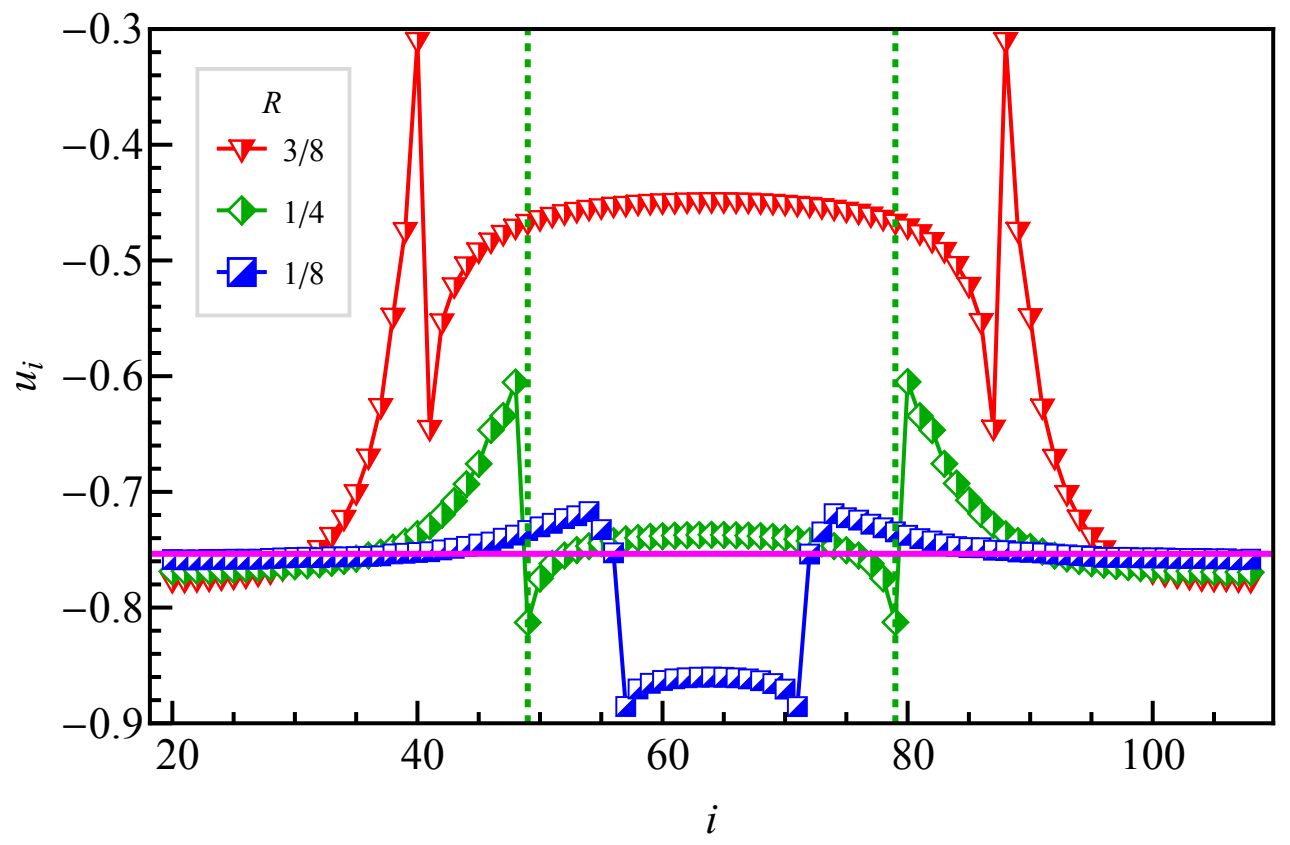

Figure 4.11: Average bond energy $u_{i}$ at site $i$ for $L=128, T=1$, and various ratios $R=w / L$. The solid magenta horizontal line indicates the equilibrium energy of the standard model at $T=1$. The dashed vertical lines are placed to demonstrate the end of the window region for the $R=1 / 4$ curve. For all curves the borders of the infinite temperature window are marked by minima in $u_{i}$ followed by discontinuous transitions to maxima outside the window. Reprinted figure with permission from N.Borchers, M. Pleimling, and R.K.P. Zia, Physical Review E, 90(6):062113, 2014. Copyright 2014 by the American Physical Society.

As the final, and perhaps strongest, characterization of the nature of the two-temperature ordered state Fig. 4.12 displays the steady state distribution $\mathcal{P}_{w}(m)$ for the normalized window magnetization $m$ as a function of $T$. This distribution provides information on how likely a certain magnetization is in the steady state. At relatively high temperatures, such as $T=2$, the histogram takes on the expected shape of a distribution centered around $m=0$, indicative of a disordered configuration and similar to the shape observed for the standard 
equilibrium system, see Fig. 3.6, as well as for the $2 J$ model, see Fig. 3.9. As the temperature is lowered, however, there appears to be a smooth inversion of the distribution such that halffilled states become increasingly unlikely while wholly filled or empty states dominate. As a result $\mathcal{P}_{w}(m)$ becomes bi-modal with maxima at some temperature-dependent normalized magnetizations $\pm m$. The seemingly continuous change of the distribution as the temperature is lowered is reminiscent of the behavior expected for a continuous phase transition across some critical temperature $T_{c}$. An example of this can be found, of course, in the standard equilibrium Ising model in dimensions $d \geq 2$, where the distribution shifts in a similar manner from a distribution centered at zero magnetization towards a bi-modal distribution with maxima at the spontaneous magnetization [54]. If the crossover displayed here is indeed a continuous phase transition, there are extremely well developed theories which should govern the system's behavior at the critical point. A few of these approaches will be demonstrated in Appendix B. These approaches are ultimately insufficient for characterizing this $2 T$ model, as shall become apparent shortly.

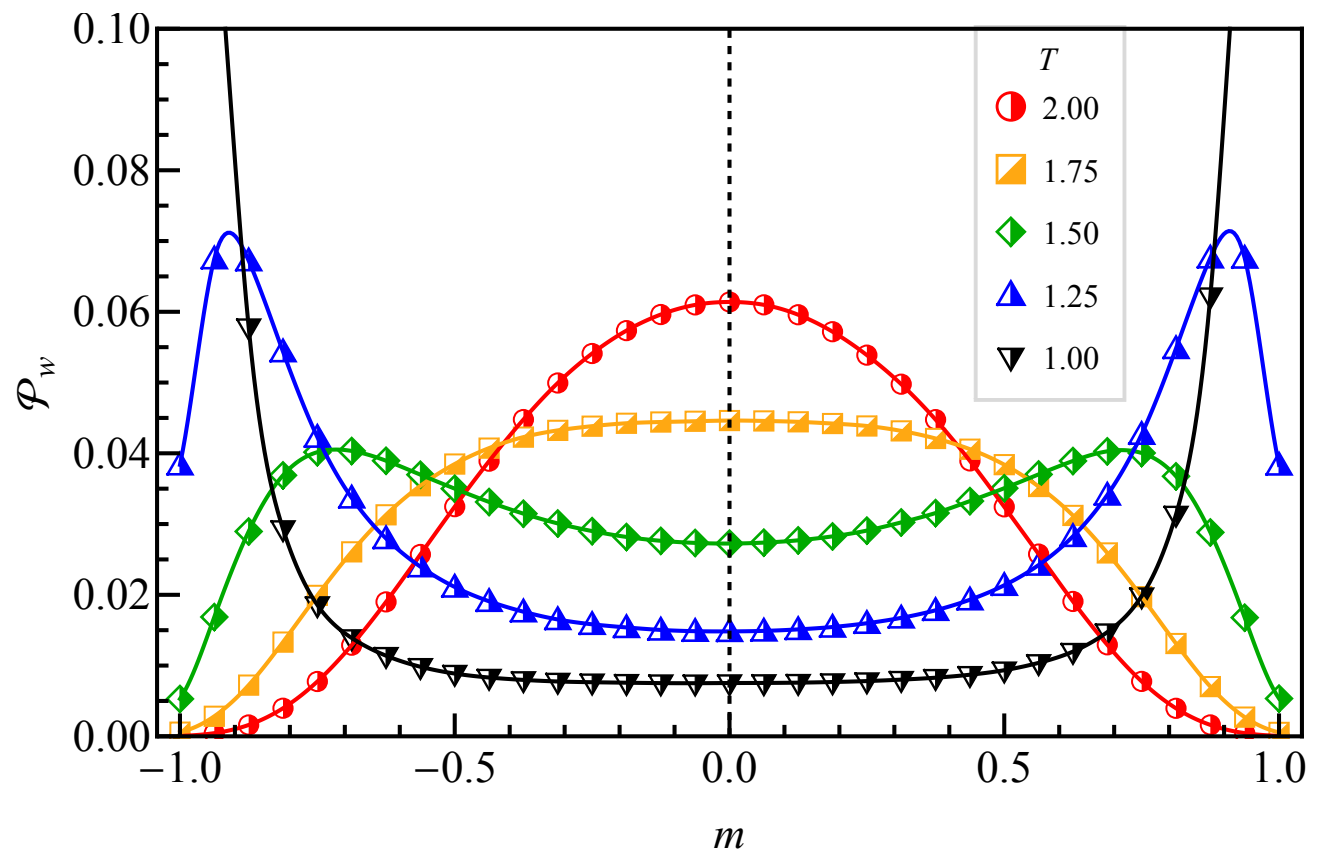

Figure 4.12: Distributions for the normalized window magnetization $m$ with system parameters $L=128$ and $R=1 / 4(w=32)$. Results for different values of $T$ are shown. Reprinted figure with permission from N.Borchers, M. Pleimling, and R.K.P. Zia, Physical Review E, 90(6):062113, 2014. Copyright 2014 by the American Physical Society.

The lattice temperature $T$ is, however, not the only parameter that may be varied to produce an apparent transition. Fig. 4.13 shows that the distribution changes considerably as the 
ratio $R=w / L$ is changed with constant $L$. The distributions evolve in a similar manner to those in Fig. 4.12 with an increase in $T$ replaced with an increase in $w$, with the notable difference that a maximum at $M=0$ exists for the red $R=3 / 8$ curve. This new peak, indicative of a more prominent disordered state, could be an indication of some form of phase coexistence in the system at larger values of $R$.

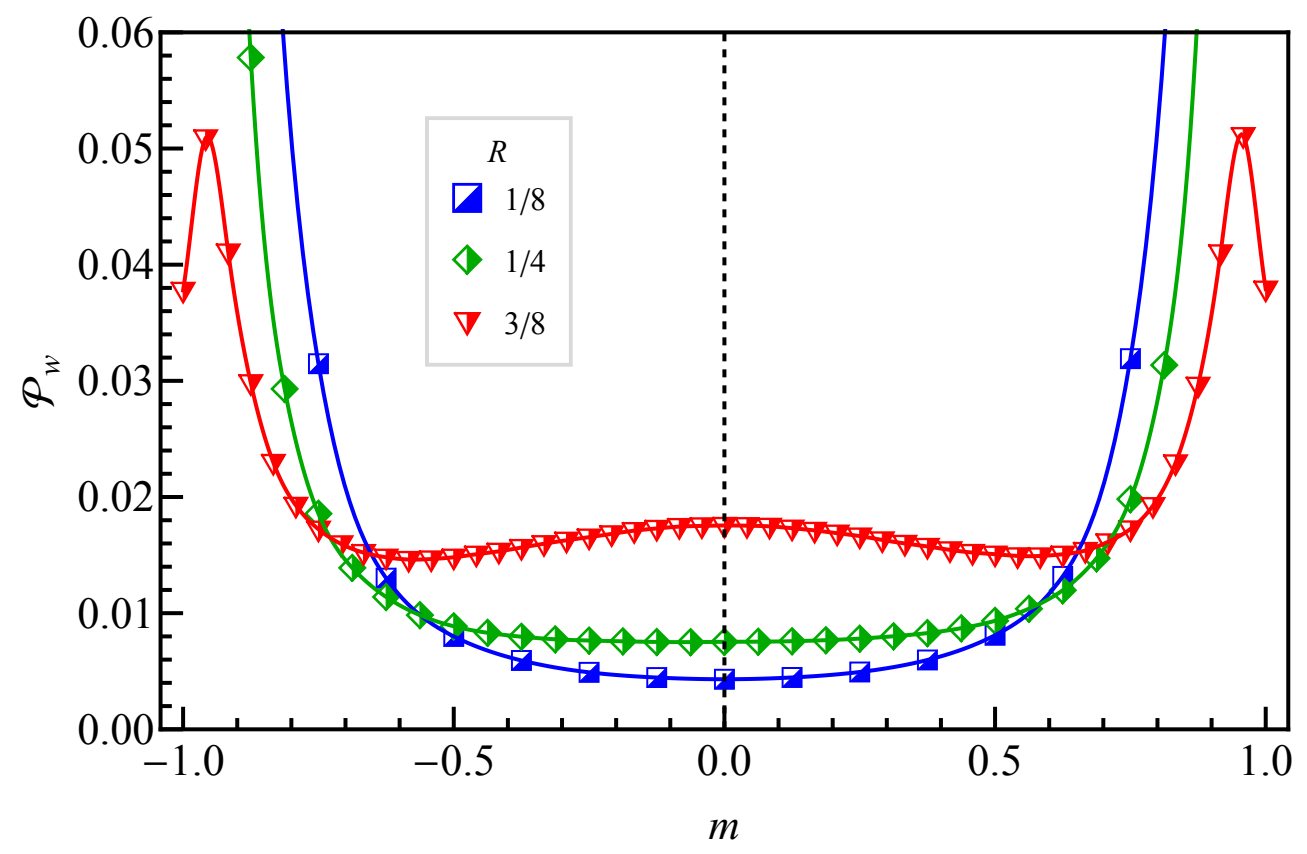

Figure 4.13: Distributions for the normalized window magnetization $m$ with $L=$ $128, T=1$, and various window sizes. Reprinted figure with permission from N.Borchers, M. Pleimling, and R.K.P. Zia, Physical Review E, 90(6):062113, 2014. Copyright 2014 by the American Physical Society.

These results indicate that the crossover between different steady states observed in the non-equilibrium two-temperature model has some of the finite-size hallmarks of a true phase transition. Whereas from the temperature-dependence one might conclude that the transition is continuous, conflicting information is obtained when varying the window size, with the appearance of additional peaks indicative of a system with phase coexistence. By utilizing the techniques discussed in Section 3.1, it is possible to explore larger system sizes and begin to resolve the apparent discrepancy between the nature of the crossover depending on which parameter is varied. Based on Figures 4.12 and 4.13, it would appear that the crossover is continuous as long as the value of $R$ is sufficiently small since the $M=0$ peak only materializes for relatively large values of $R$. However, as the system size is increased, Fig. 4.14 makes it clear that this disordered peak emerges in larger systems even at values of $T$ and $R$ in which the ordered state normally dominates. 


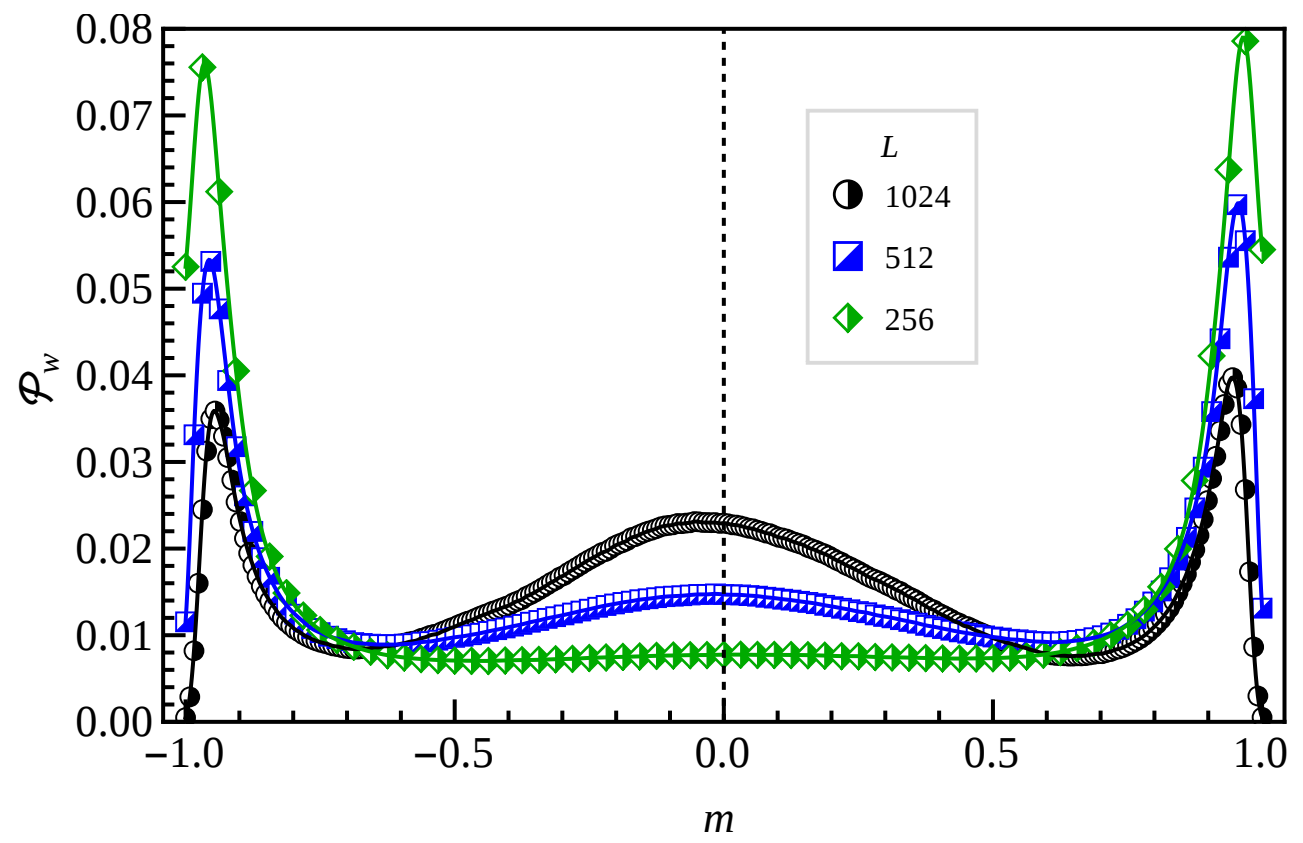

Figure 4.14: Distributions for the normalized window magnetization $m$ with $R=$ $1 / 4, T=1$, and various system sizes. The curves are generated by averaging over 200 independent realizations, each with $2^{30} \mathrm{MCS}$ of relaxation and $2^{20}$ simulation MCS.

As mentioned above, the existence of peaks near $m=0$ and $m= \pm 1$ is reminiscent of a system exhibiting phase coexistence. It is, however, impossible for both phases to exist simultaneously within the window. Imagine an arrangement where this was the case, with say half the window ordered and half disordered. Since $T_{w}=\infty$ within the window, particles and holes move via free diffusion. Compared to the time scales set by the evolution of the spins within the low temperature region of the lattice, any separation between ordered and disordered phases within the window would dissolve nearly instantaneously. The three peaks on the curves of Fig. 4.14 must therefore represent a form of temporal coexistence, where the system splits time amongst states in which the window is ordered and disordered. This interpretation fits in perfectly with the results shown at the end of Section 4.2, in particular Fig. 4.9. There it was shown that the steady state for larger system sizes is actually comprised of two independent states of comparable stability each with a distinct overall energy density. Interpreting these results together, it is then clear that the peaks of the distributions reflect the underlying bistability of the system, and that the two unique energy densities come from states in which the window is either ordered or disordered.

Based on the small sample size presented by Fig. 4.9, the bistable states would appear to 


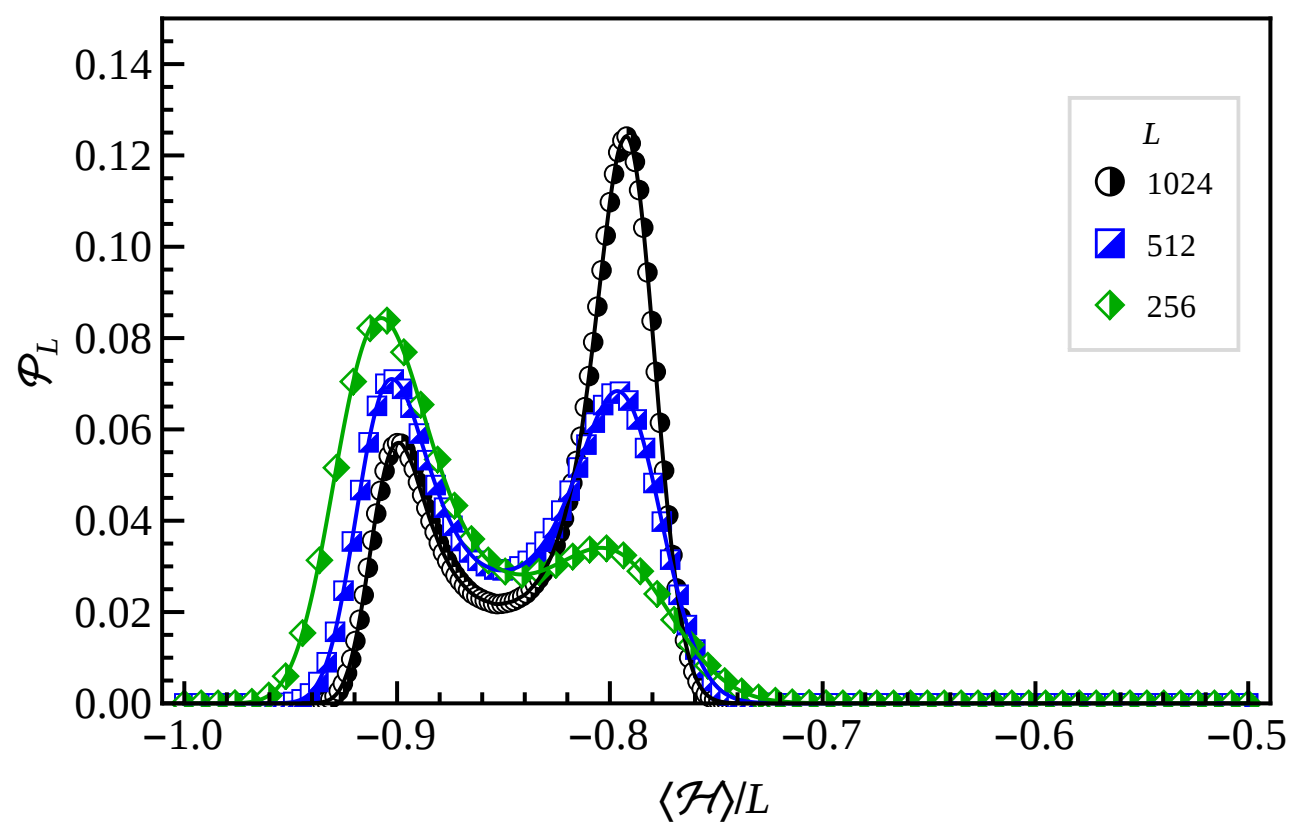

Figure 4.15: Distributions for the average system energy density $\langle\mathcal{H}\rangle / L$ with $R=$ $1 / 4, T=1$, and various system sizes. The curves are generated by averaging over 200 independent realizations, each with $2^{30}$ MCS of relaxation and $2^{20}$ simulation MCS.

be equally likely. Fig. 4.15 explores this question further by constructing histograms of the average energy density for a few of the larger values of $L$ which have been considered. These distributions are unsurprisingly bimodal, again as a reflection of the underlying bistability present within the steady state. From this figure it is clear that the two states, while present in the steady state of all system sizes considered, are not necessarily equally prevalent. It is noteworthy that the balance between these states depends strongly on the overall system size, with the lower energy ordered state growing in stature relative to the disordered state as the system size is reduced. At $L=512$ the two states are found with nearly equal frequency, and it seems to be a bit of good fortune that such a feature is found within a computationally accessible system size. These results lend credence to the notion that the ordered state is a consequence of finite-size effects, only emerging as the system size is reduced. The disordered state, which has already been demonstrated to be the natural behavior for an equilibrium system at infinite temperature, appears as if it would dominate at ever larger system sizes. But it must be cautioned the results shown are only at the single value of $T$ and $R$. Before true conclusions can be drawn on how these effects would manifest themselves in the thermodynamic limit it would be necessary to consider how variations in these parameters influence the balance of these states. 

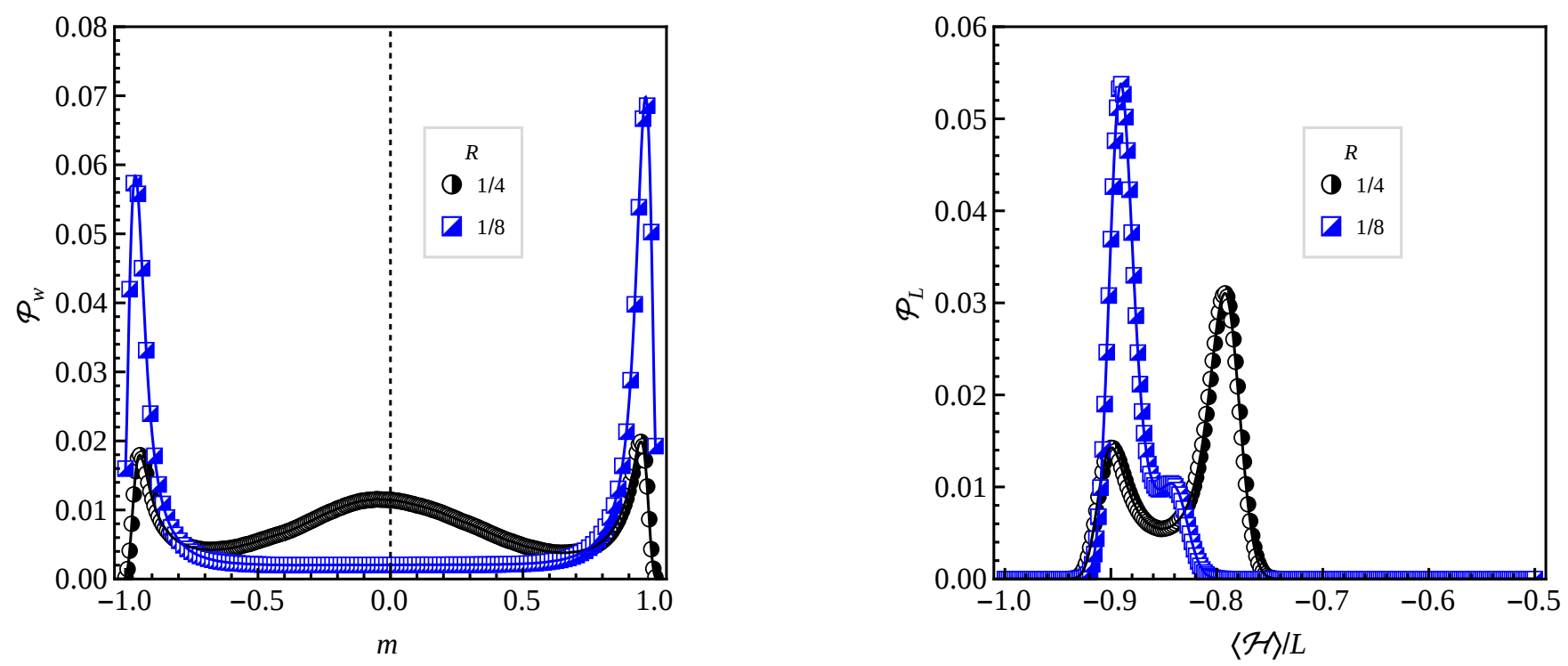

Figure 4.16: Left: Distributions for the normalized window magnetization $m$ with $L=1024, T=1$, and two values of $R$. Right: The same as the left, but for the average energy density $\langle\mathcal{H}\rangle / L$ instead. Both curves are generated by averaging over 200 independent realizations, each with $2^{30} \mathrm{MCS}$ of relaxation and $2^{20}$ simulation MCS.

Indeed, it can be immediately shown that variations in $R$ influence the balance between the states. In the largest of these systems, $L=1024$, the disordered state shows the greatest amount of influence based on all the preceding evidence. While this occurs with system parameters for which smaller systems are dominated by the ordered state, i.e. $R=1 / 4$ and $T=1$, this trend can still be reversed by decreasing the value of $R$ even further. In Fig. 4.16 it is shown that the ordered state is recovered in the $L=1024$ system. From this it seems that the ordered state can persist even at larger system sizes, though it would appear to require $R$ to depend on $L$ in some non-linear manner, e.g. $R=\alpha L^{\nu}$ where $\alpha$ and $\nu$ are constants and $\nu<1$. Whether this is the case or not will require a more rigorous finite-size analysis.

\subsection{Small system analysis}

As in the case with the equilibrium models, systems with small values of $L$ present an opportunity to solve the master equation explicity. By exploring small versions of the $2 T$ model, it is again the hope that the finite-size effects, while unavoidably large, will still allow features of larger systems to be discerned and understood in the context of the smaller systems. In fact, there are no compelling reasons to believe this will be the case, and this exercise is done due to its accessibility and with a good measure of hopefulness. However, 
Table 4.1: The 10 energetic configurations of the $2 \mathrm{~T}$ model for $L=6, w=2$, and $\mathcal{M}=0$. The bonds which fall within the window are contained within the red vertical lines of the configurations. The cell colors correspond to the node colors in Fig. 4.17.

\begin{tabular}{|c|ccc|cc|cc|c|}
\hline Number & \multicolumn{5}{|c|}{ Configuration } & Energy \\
\hline 1 & - & $\mid$ & - & - & $\mid$ & - & -2 \\
2 & $\mid$ & - & - & $\mid$ & - & - & -2 \\
3 & - & - & $\mid$ & - & - & $\mid$ & -2 \\
\hline 4 & - & $\mid$ & $\mid$ & $\mid$ & - & $\mid$ & 2 \\
5 & $\mid$ & - & $\mid$ & $\mid$ & $\mid$ & - & 2 \\
6 & - & $\mid$ & - & $\mid$ & $\mid$ & $\mid$ & 2 \\
7 & $\mid$ & $\mid$ & $\mid$ & - & $\mid$ & - & 2 \\
8 & $\mid$ & $\mid$ & - & $\mid$ & - & $\mid$ & 2 \\
9 & $\mid$ & - & $\mid$ & - & $\mid$ & $\mid$ & 2 \\
\hline 10 & $\mid$ & $\mid$ & $\mid$ & $\mid$ & $\mid$ & $\mid$ & 6 \\
\hline
\end{tabular}

it will be seen that, despite the inherent limitations, there are in fact insights that can be made.

Proceeding with $L=6$ allows direct comparison with the equilibrium results of Section 3.3, while setting the window to $w=2$ allows for parallels with Section 3.2. First, the states are enumerated in Table 4.1, which is identical to Table 3.1 save for the vertical red lines indicating the edges of the infinite temperature window. This should be contrasted with Table 3.2, where the center two bonds are replaced with zero indicating that $J=0$ for interactions at these sites. Here already a fundamental difference between the $2 J$ and $2 T$ model can be observed. Despite the fact that both a null coupling constant and infinite temperature result in free diffusion, the former accomplishes this by shifting the energy levels of the system such that transitions which formerly required energy input from the reservoir now happen spontaneously. In this way, detailed balance is never broken in the $2 J$ model since the Metropolis rates can simply be updated to reflect the new energy levels of the model.

In the $2 T$ model, however, the configuration network remains unchanged from the standard equilibrium structure found in the left portion of Fig. 3.10. Rather, the rates between configurations involving exchanges within the window are directly modified such that the probability of an exchange is one regardless of $\Delta \mathcal{H}$. This immediately precludes both the Boltzmann distribution and detailed balance, as the Metropolis criteria cannot be met for $W_{i}^{j}=W_{j}^{i}$ if $\mathcal{H}_{i} \neq \mathcal{H}_{j}$. The edges for these new unity rates have been highlighted in red within the configuration network in Fig. 4.17, and represent a pathway for energy to flow from the infinite temperature reservoir into the system. 

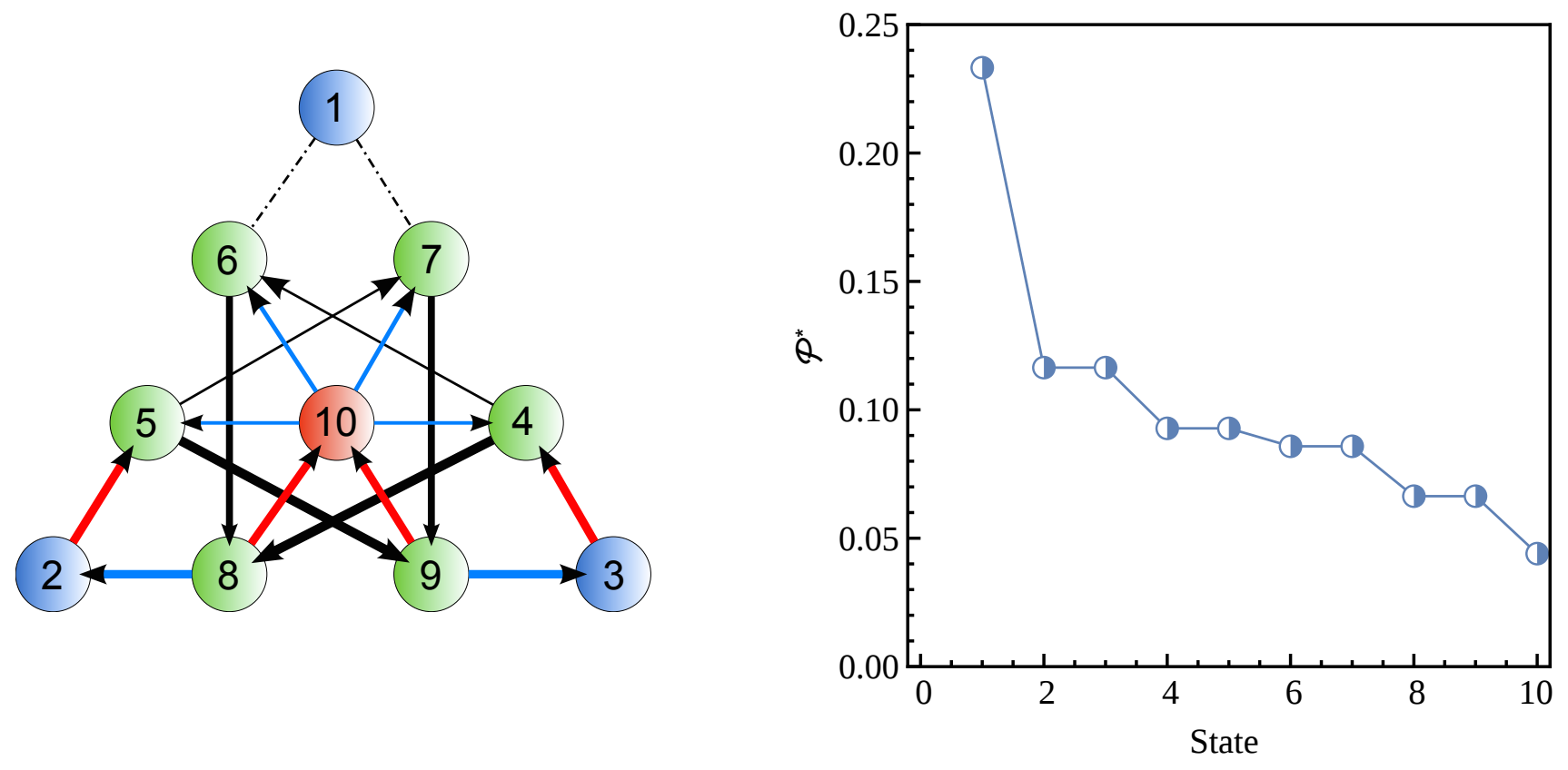

Figure 4.17: The left image displays the configuration network for Kawasaki dynamics acting on $L=6, \mathcal{M}=0$, and $w=2$. The numbers correspond to the states indicated in Table 3.1, while the colors refer to the corresponding energy level. The directed edges between the states indicate the direction of the probability current, while the color corresponds to the source of energy gain or loss. Red edges indicate energy gained from the infinite temperature reservoir, while blue indicate energy deposited into the low temperature reservoir. Dashed lines indicate zero current. In the right plot, the calculated values of $P^{*}$ for this system at $T=4$ are shown.

An immediate consequence of this breaking of detailed balance is the persistence of probability currents within the steady state. These currents, defined as

$$
K_{i}^{* j} \equiv W_{i}^{j} P_{j}^{*}-W_{j}^{i} P_{i}^{*}
$$

give a measure for how often the transition $\{\sigma\}_{i} \rightarrow\{\sigma\}_{j}$ occurs relative to the reverse. This quantity has often been suggested as the principal characteristic of NESS [7], one which may be used to measure how far from equilibrium a NESS is [55]. By solving for $P^{*}$ (right image in Fig. 4.17) via the method discussed in Section 3.3, it is straightforward to then determine $K_{i}^{* j}$. These currents are indicated in the configuration network by arrows between the states, with the direction and thickness of the arrows corresponding to the sign and magnitude respectively. Additionally, color has been added to the edges to indicate energy gains and losses by the system. Red edges represent currents bringing energy from the infinite temperature reservoir into the system, while blue edges represent energy lost from the 
system into the lower temperature reservoir. The dashed lines, which conspicuously connect state 1 to the rest of the system, represent paths along which no current flows. While it is difficult to immediately discern the significance of these currents to the observed behavior of the steady state, they play a crucial role when considering both the flow of heat through the system and the generation of entropy.

Before considering these aspects, it is worth focusing more attention on plot of $P^{*}$ itself in Fig. 4.17. The first thing to notice is that the distribution is strongly peaked at state 1 even at this relatively high temperature $(T=4)$. As the temperature is lowered further, this configuration absolutely dominates the distribution, approaching values for $P^{*}$ above 0.99 near absolute zero. Even at $T=1$ the system spends significantly more time in configuration 1 than all other configurations combined. Referring back to Table 4.1 it can be seen that this is precisely the configuration for which the system is ordered over the window (no unsatisfied bonds within the window). Due to the extremely small size of the system, it is difficult to say if this represents an indication of the ordered phase forming at low values of $T$, but it is compelling evidence that small systems might exhibit some important features of the 'freezing-by-heating' phenomenon.

The distribution has more structure than either of the equilibrium Boltzmann distributions (Figs. 3.10 and 3.11), but it is interesting to observe that many of the states share identical values for $P^{*}$. This is a clue that a symmetry in the problem has not been fully exploited. In the Boltzmann distribution all states with the same energy have the same value of $P^{*}$. While the use of the Metropolis algorithm, in conjunction with detailed balance, ensured that the Boltzmann distribution would emerge for the equilibrium models considered earlier, the fact that all equal energy configurations share the same value of $P^{*}$ can also be seen as a consequence of the translational symmetry inherent in the equilibrium model. By enforcing periodic boundary conditions the model exhibits this symmetry naturally, and therefore a shift of coordinates should not change the observed physics. Since all equal energy configurations can be constructed from one another via a simple translation these states are essentially equivalent and must have equal probabilities in the steady state.

The $2 T$ model, however, breaks this symmetry via the location of the infinite temperature window. It should come as no surprise then that the Boltzmann distribution couldn't possibly suffice for the $2 T$ model. Regardless, there is still an important symmetry in the model which persists even with the introduction of the window. This is the parity, or mirror, symmetry about the center of the window. This then explains the four sets of states which share the same value of $P^{*}$, since each pair is related under this symmetry. The two remaining configurations (state 1 and 10) are unique in that they are their own parity partner. This symmetry then allows the configuration space to be further reduced to 6 unique state, a process which is visualized in Fig. 4.18 by a folding of the now familiar configuration network layout. The ordering of the states for this network should finally be clear.

This reduction of the configuration network illuminates several features of the probability currents and $P^{*}$ distribution in Fig. 4.17. This figure has been reproduced in terms of the 

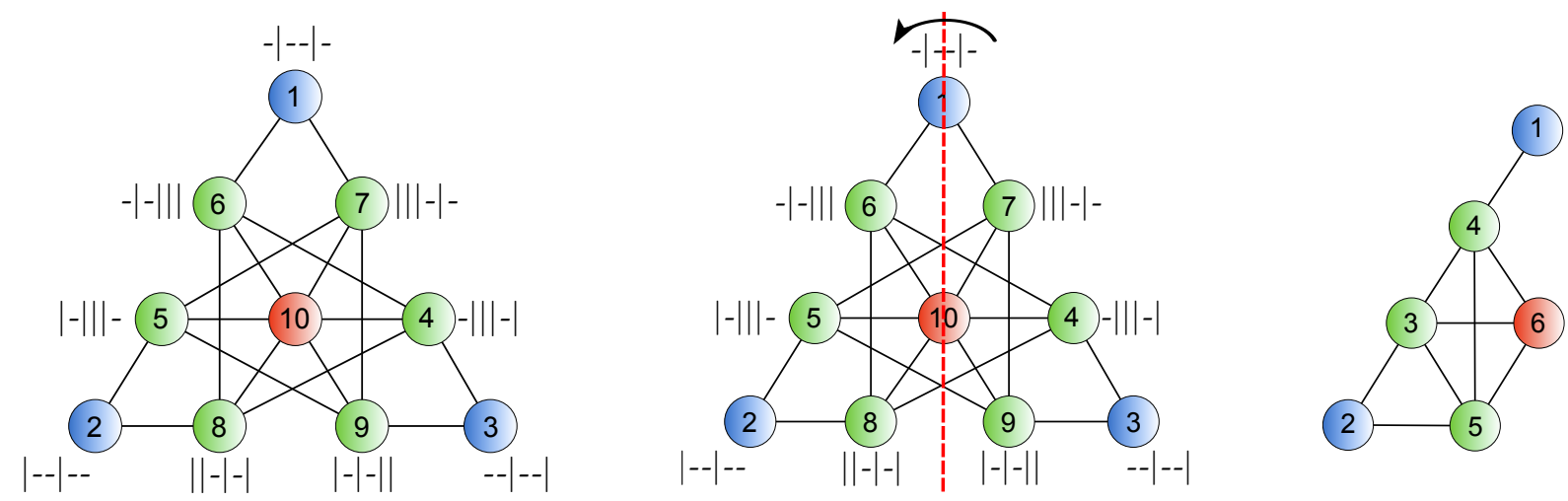

Figure 4.18: Here the configuration network for Kawasaki dynamics acting on $L=6, \mathcal{M}=0$, and $w=2$ is shown again to emphasize the underlying parity symmetry which exists within the model. The left image shows configuration space with the bond representation of the state mapped to each node (excepting the central state of maximum energy which is it's own mirror image) to emphasize the parity symmetry in the model. The central image shows how the symmetric states can be folded together, while the rightmost image displays the reduced network representation.

reduced network in 4.19. One immediate consequence which is clear from this perspective is the lack of probability current around configuration 1. Since this state is its own parity partner, the folding in Fig. 4.18 reduces the degree of this node from two to one. With a single edge connecting this configuration to the rest of the model, there is no way for probability to flow through this state in the steady state. Therefore, the current must be zero. Another thing this perspective provides is an emphasis of the heat flow cycles through the system. As mentioned previously, the heat flow through the system can be visualized via the current loops on the network. Heat flows into the model from the window along red directed edges, and flows out to the cooler reservoir via the blue edges. The system may then 'reset' by moving along the black edges, returning to the initial state and completing the cycle. The transitions along the black edges require no energy input since these states are all at the same energy, and therefore can be considered to occur spontaneously. There are three simple, repeatable cycles of length three that can be identified; $\{2,3,5\},\{5,6,3\},\{5,6,4\}$. The fact that the largest current in the system exists for the spontaneous transition $3 \rightarrow 5$ can now be understood since this path is contained within two of these heat flow cycles.

A final quantity which may be explored with $P^{*}$ known is the Gibbs entropy of the system, $S_{s y s}$, which is defined in the standard way as

$$
S_{\text {sys }}=\sum_{i} P_{i}^{*} \log P_{i}^{*}
$$

By solving for $P^{*}$ via master equation, it is possible to consider the value for $S_{\text {sys }}$ over a range 

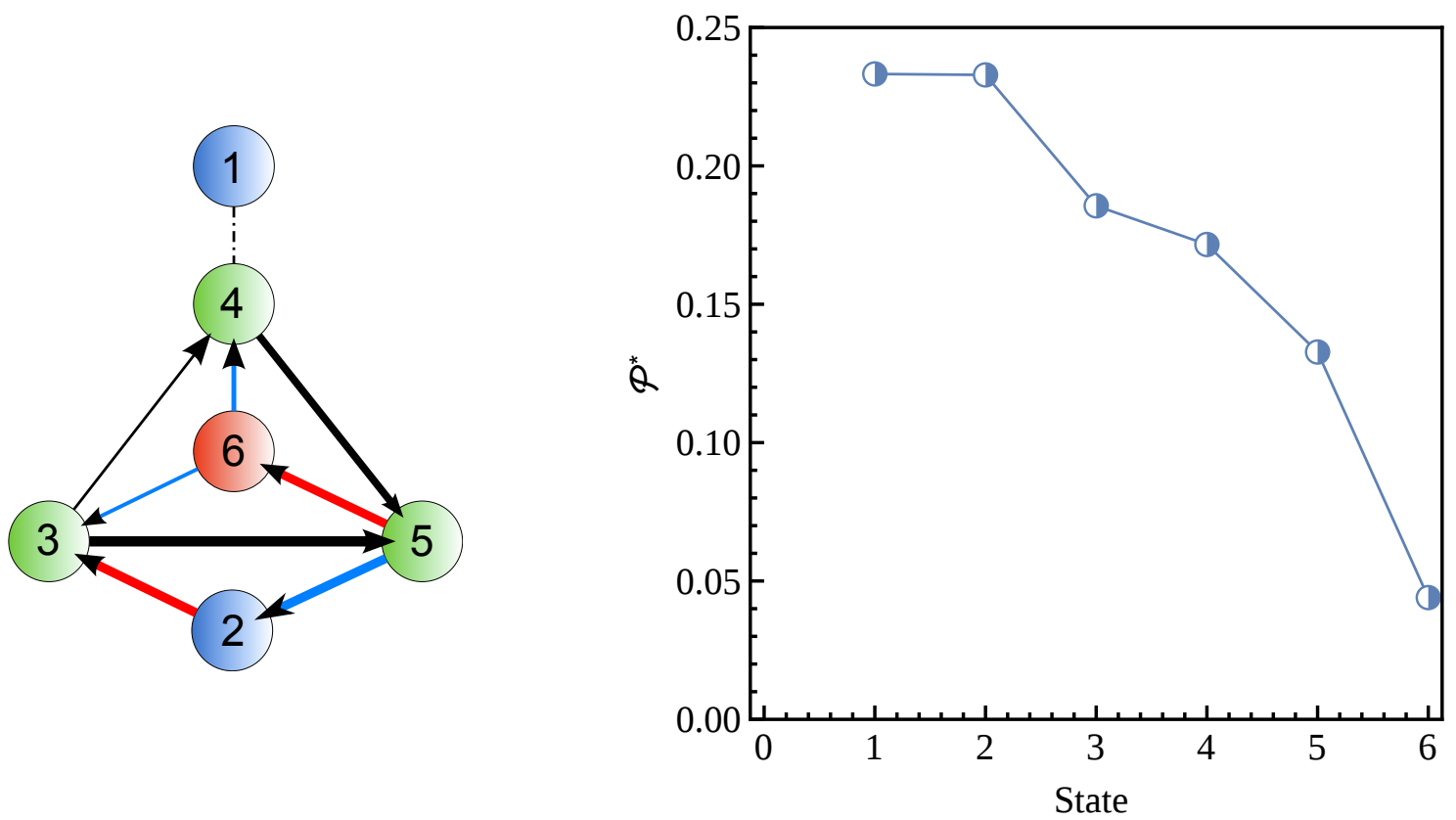

Figure 4.19: The left image displays the parity reduced configuration network for Kawasaki dynamics acting on $L=6, \mathcal{M}=0$, and $w=2$. The colors still refer to the energy levels indicated in Table 3.1. The directed edges between the states indicate the direction of the probability current, while the color corresponds to the source of energy gain of loss. Red edges indicate energy gained from the infinite temperature reservoir, while blue edges indicate energy deposited into the low temperature reservoir. Dashed lines indicate zero current. In the right plot, the calculated values of $P^{*}$ for this system at $T=4$ are shown.

of $T$ values. These curves are shown in Fig. 4.20. The equilibrium curve - shown in magenta is provided for reference. The most striking feature of this figure is the fact that at sufficiently low temperatures $S_{\text {sys }}$ is reduced below the equilibrium level for the two smaller windows $(w=1$ and $w=2)$. This is consistent with the idea that an ordered state emerges within the system when both $T$ and $w$ are sufficiently small. It is still counterintuitive that the entropy of the system can be lowered by coupling a portion of the system to an infinite temperature, particularly since an infinite temperature typically pushes $P^{*}$ towards a uniform distribution over the states, i.e. energy differences in the system are unimportant at infinite temperature. Such a uniform distribution is precisely the $P^{*}$ which maximizes entropy via eqn. 4.2 , which is the reason all the curves reach their maximum at $\exp (-4 / T)=1$ in the figure. For the $w=2$ curve, not only does $S_{\text {sys }}$ go below the equilibrium value at low temperatures, but it in fact approaches zero as $T$ does. While at first glance remarkable, this is is simply a consequence of the distribution approaching a Kronecker delta at state 1. The system is, from an information theoretic perspective [56], reaching a maximum of information content, i.e. the system will definitely be found in state 1, and therefore the entropy is minimized at 
zero.

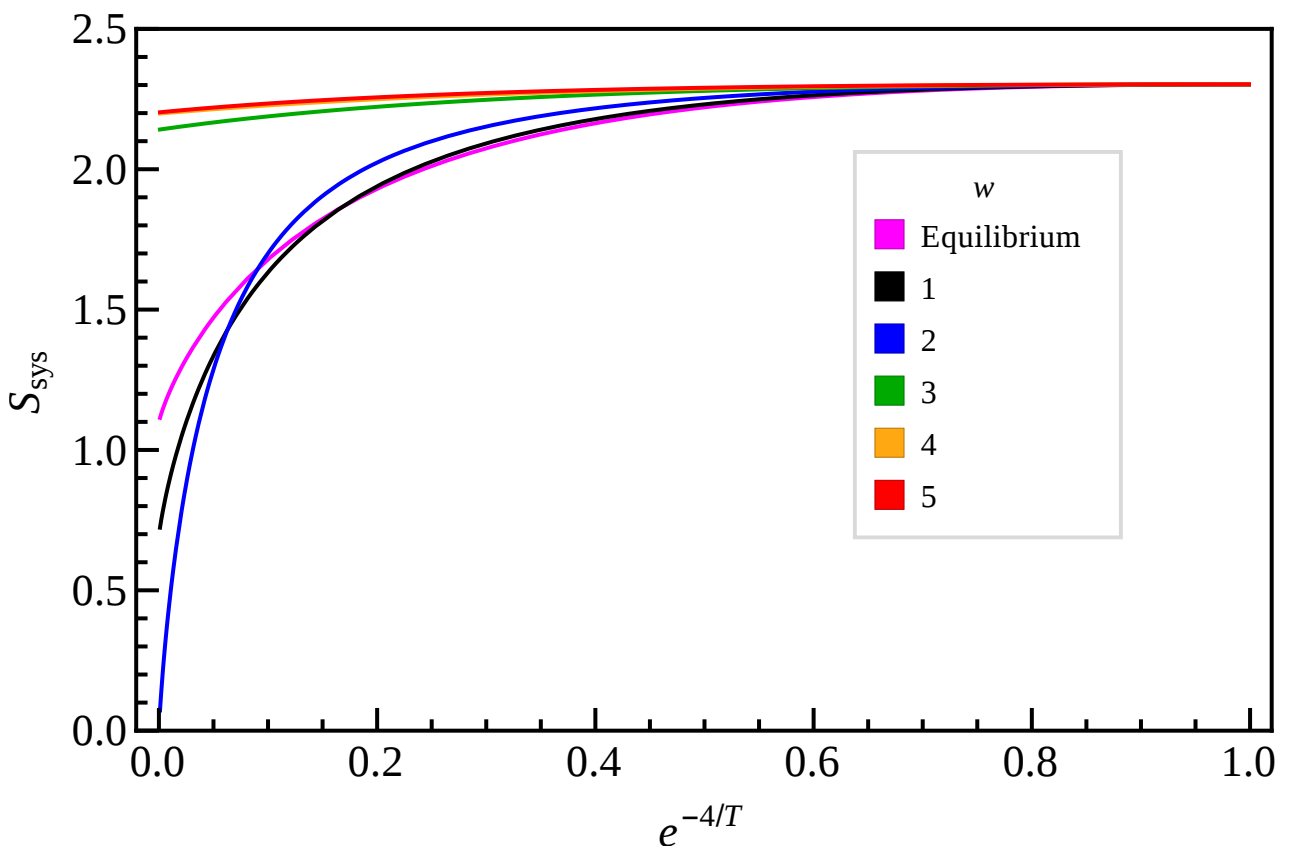

Figure 4.20: System entropy $S_{\text {sys }}$ versus temperature scaled as $\exp (-4 / T)$ for the $L=6$ model at a variety of window sizes. Note the system entropy for $w=1$ and $w=2$ is lower than the equilibrium value for low values of $T$

While exploring the system entropy is interesting in its own right, an exhaustive consideration of entropy must include the entropy generated within the environment, $S_{\text {env }}$, due to the transfer of heat from the hot to the cold reservoir. In addition, it would be interesting to examine how the Gibbs entropy in eqn. 4.2 compares with a calculation of the standard thermodynamic entropy,

$$
\Delta S=\frac{\Delta Q}{T}
$$

When combined with the stationary currents calculated with eqn. 4.1, this could be used to calculate the average entropy produced per unit time. This is, however, beyond the scope of this current study. 


\section{Chapter 5}

\section{Conclusion}

Gaining a comprehensive understanding of non-equilibrium processes remains an enormous task, due to the wealth and diversity of phenomena that emerge far from equilibrium. Far from having a general theoretical framework, we proceed by gathering valuable insights through the detailed analysis of simple model systems.

To this end, here we have studied a version of the conserved one-dimensional Ising model on a ring with a minimal breaking of detailed balance. In this version of the two temperature model, two sectors of the system are coupled to different heat baths: one with infinite temperature for exchanges within a window of size $w$, and one at some temperature $T$ for the remainder of the ring. Since spin exchanges within $w$ are indistinguishable from an Ising model with $J=0$ coupled to the same $T$, this $2 J$ model, in thermal equilibrium, was also considered. The difference between these models lies in detailed balance being violated in the former, but only for two pairs of exchanges at the 'window edges.' This minimal modification at the interfaces entails huge changes in the physical properties of the system, yielding a remarkable 'freezing-by-heating' ordered state. Using extensive numerical simulations we characterized this state through a variety of quantities, most notably the spin-spin correlations as well as the probability distribution of the normalized window magnetization. Varying the temperature $T$ or the window size yields a transition between a state with a highly ordered window and a state where the window is disordered.

The finite-size signatures of this transition are ambiguous, and more work needs to be done to clarify the nature of this 'transition.' Indeed, we are aware that long range correlations are well known to emerge in such driven diffusive systems (breaking detailed balance in Kawasaki dynamics [27]), and so, the phenomenon observed here may be due to finite-size effects. Further support for the possibility that finite-size effects play an essential role in the emergence of the ordered state are provided by the bistable nature of the steady state at larger values of $L$. In the case of larger systems $(L \geq 512)$, the system splits time between the ordered and disordered state, the balance of which is determined by both $L$ and $T$. At a fixed value of $T$, it was shown that the disordered state increasingly dominates as $L$ is 
increased. As $T$ is decreased in response the ordered behavior is recovered, but it is unclear if the ordered state could persist in the thermodynamic limit for any $T>0$. In particular, it is reasonable to conjecture that, if two semi-infinite systems coupled to different baths were joined at one point, the behavior far in the bulk of either system would be 'normal,' while long range correlations induce extended boundary layers on either side of the junction. On an extremely large ring (with fixed $R$ ), there would be two such junctions, but widely separated. Yet, when the ring size is small enough, the two boundary layers will 'interact' and may produce the ordered states observed here. Substantial progress in our understanding of these systems therefore relies on gaining a full understanding of how long range correlations can lead to apparent long range order.

In addition to the steady state properties of this system, dynamical properties were revealed by studying how the system relaxes towards the steady state from a variety of initial preparations. It was revealed that the time scale for initiation of the steady state is proportional to $w$ to the third power. That this relationship mirrors the one for domain growth in the equilibrium Ising model with spin exchange dynamics is a hint that domain growth outside the window is a key mechanism for the existence of the ordered state. It is possible that the long range correlations originating at the window boundaries allow sufficiently large domains outside the window to interact and converge on the window. The relaxation process also provides insight into the bistable steady state through the appearance of the metastable state which precedes it. This state, which initially appeared to hinder efforts to deeply probe the steady state, is a yet another manifestation of bistability. When the system is quenched to a low temperature the disordered half of the steady state is encountered first and persists for some time prior to the ordered configuration arising. That this feature is more prominent in the relaxation curves of the larger system sizes is consistent with the established qualities of the bistability.

Finally, by performing explicit calculations of the master equation it was possible to determine exactly $P^{*}$ for an extremely small system. Though necessarily dominated by finite-size effects, the solution of $P^{*}$ still indicates that the system would enter a state with an ordered window at low temperatures. Of course, if finite-size effects are ultimately the source of the ordered state this would not necessarily come as a surprise. On a more fundamental level, it was also revealed that, even though the translational symmetry of the standard model is broken by the inclusion of a the window, the parity symmetry remains and is still respected by the distribution. The degeneracy of the energy levels within the equilibrium Boltzmann distribution is 'lifted' by the lack of translational symmetry, but another degeneracy exists due to the parity symmetry. Unfortunately, aside from this symmetry and the spin flip symmetry, it appears as if each state must be treated independently when considering the corresponding value of $P^{*}$. Examining the configuration space of the small system and mapping the probability currents to this network, it is clear that the effects of the infinite temperature window are not applied in a fully symmetric manner, i.e. states which are isomorphic to one another within in terms of degree, position, and energy within Fig. 4.17 do not have the same currents originating from the window flowing through them. This further 
confirms the unique character of each of these states.

In all cases, we believe that further work on simple model systems such as this one is likely to produce novel and surprising phenomena, as well as to provide insights into how an overarching framework for non-equilibrium statistical mechanics can be established. Such a framework would be of immeasurable utility and would alleviate the current state of affairs in which each model must be treated independently. Whether such a framework is possible, and what form it might take, are worthy questions with no clear answers. For instance, finding a non-equilibrium equivalent of the free energy, a quantity of central importance in equilibrium statistical mechanics and thermodynamics, may be one avenue of pursuit. But any analogous non-equilibrium quantity will likely be considerably different, if only for the reason that the total energy of a given configuration has a significantly reduced influence on the probability of a given state. While such pursuits are noble in their aims, it should never be forgotten how exceptional the equilibrium condition truly is. It could very well be that the techniques established for equilibrium systems have no more use (analogous or otherwise) for non-equilibrium systems than does potential theory for non-conservative forces. Despite this, we hope that effort will continue in this direction and will one day illuminate fundamental principles of these fascinating systems. 


\section{Bibliography}

[1] N. Borchers, M. Pleimling, and R.K.P. Zia. Nonequilibrium statistical mechanics of a two-temperature Ising ring with conserved dynamics. Physical Review E, 90(6):062113, 2014.

[2] J. Sethna. Statistical mechanics: entropy, order parameters, and complexity, volume 14. Oxford University Press, 2006.

[3] M. Kardar. Statistical physics of particles. Cambridge University Press, 2007.

[4] U. Seifert. Stochastic thermodynamics, fluctuation theorems and molecular machines. Reports on Progress in Physics, 75(12):126001, 2012.

[5] T. Chou, K. Mallick, and R.K.P. Zia. Non-equilibrium statistical mechanics: from a paradigmatic model to biological transport. Reports on Progress in Physics, 74(11):116601, 2011.

[6] T.L. Hill. Studies in irreversible thermodynamics iv. diagrammatic representation of steady state fluxes for unimolecular systems. Journal of Theoretical Biology, 10(3):442459, 1966.

[7] R.K.P. Zia and B. Schmittmann. Probability currents as principal characteristics in the statistical mechanics of non-equilibrium steady states. Journal of Statistical Mechanics: Theory and Experiment, 2007(07):P07012, 2007.

[8] S.G. Brush. History of the Lenz-Ising model. Reviews of Modern Physics, 39(4):883, 1967.

[9] P.C. Hohenberg and B.I. Halperin. Theory of dynamic critical phenomena. Reviews of Modern Physics, 49(3):435, 1977.

[10] W. Lenz. Beitrag zum Verständnis der magnetischen Erscheinungen in festen Körpern. Z. Phys., 21:613-615, 1920.

[11] E. Ising. A contribution to the theory of ferromagnetism. Z. Phys, 31(1):253-258, 1925. 
[12] W. Heisenberg. Zur Theorie des Ferromagnetismus. Zeitschrift für Physik, 49(9-10):619636, 1928.

[13] B.A. Cipra. An introduction to the Ising model. American Mathematical Monthly, 94(10):937-959, 1987.

[14] R.J. Glauber. Time-dependent statistics of the Ising model. Journal of Mathematical Physics, 4:294, 1963.

[15] K. Kawasaki. Kinetic equations and time correlation functions of critical fluctuations. Annals of Physics, 61(1):1-56, 1970.

[16] C. Godrèche and A.J. Bray. Nonequilibrium stationary states and phase transitions in directed Ising models. Journal of Statistical Mechanics: Theory and Experiment, 2009(12):P12016, 2009.

[17] C. Godreche. Dynamics of the directed Ising chain. Journal of Statistical Mechanics: Theory and Experiment, 2011(04):P04005, 2011.

[18] C. Godrèche. Rates for irreversible Gibbsian Ising models. Journal of Statistical Mechanics: Theory and Experiment, 2013(05):P05011, 2013.

[19] C. Godreche and M. Pleimling. Dynamics of the two-dimensional directed Ising model in the paramagnetic phase. Journal of Statistical Mechanics: Theory and Experiment, 2014(5):P05005, 2014.

[20] T. Lee and C. Yang. Statistical theory of equations of state and phase transitions. ii. lattice gas and Ising model. Physical Review, 87(3):410, 1952.

[21] N. Metropolis, A.W. Rosenbluth, M.N. Rosenbluth, A.H. Teller, and E. Teller. Equation of state calculations by fast computing machines. The Journal of Chemical Physics, 21:1087, 1953.

[22] K.E. Bassler and B. Schmittmann. Critical dynamics of nonconserved Ising-like systems. Physical Review Letters, 73(25):3343, 1994.

[23] G. Grinstein, C. Jayaprakash, and Y. He. Statistical mechanics of probabilistic cellular automata. Physical Review Letters, 55(23):2527, 1985.

[24] U.C. Täuber and S. Diehl. Perturbative field-theoretical renormalization group approach to driven-dissipative bose-einstein criticality. Physical Review X, 4(2):021010, 2014.

[25] S. Trimper. Master equation and two heat reservoirs. Physical Review E, 74(5):051121, 2006.

[26] T.L. Hill. On the one-dimensional steady-state Ising problem. The Journal of Chemical Physics, 76(2):1122-1127, 1982. 
[27] C. Domb, R.K.P. Zia, B. Schmittmann, and J.L. Lebowitz. Statistical mechanics of driven diffusive systems, volume 17. Academic Press, 1995.

[28] P.L. Garrido and J. Marro. Ising models with anisotropic interactions: Stationary nonequilibrium states with a nonuniform temperature profile. Physica A: Statistical Mechanics and its Applications, 144(2):585-603, 1987.

[29] H.W.J. Blote, J.R. Heringa, A. Hoogland, and R.K.P. Zia. Critical properties of nonequilibrium systems without global currents: Ising models at two temperatures. Journal of Physics A: Mathematical and General, 23(16):3799, 1990.

[30] Z. Rácz and R.K.P. Zia. Two-temperature kinetic Ising model in one dimension: Steadystate correlations in terms of energy and energy flux. Physical Review E, 49(1):139, 1994.

[31] P. Tamayo, F.J. Alexander, and R. Gupta. Two-temperature nonequilibrium Ising models: Critical behavior and universality. Physical Review E, 50(5):3474, 1994.

[32] B. Schmittmann and F. Schmüser. Stationary correlations for a far-from-equilibrium spin chain. Physical Review E, 66(4):046130, 2002.

[33] M. Mobilia, R.K.P. Zia, and B. Schmittmann. Complete solution of the kinetics in a far-from-equilibrium Ising chain. Journal of Physics A: Mathematical and General, 37(32):L407, 2004.

[34] V. Lecomte, Z. Rácz, and F. van Wijland. Energy flux distribution in a twotemperature Ising model. Journal of Statistical Mechanics: Theory and Experiment, 2005(02):P02008, 2005.

[35] I. Mazilu and H.T. Williams. Exact energy spectrum of a two-temperature kinetic Ising model. Physical Review E, 80(6):061109, 2009.

[36] B. Renklioglu and M.C. Yalabik. Non-equilibrium phase transitions in the twotemperature Ising model with Kawasaki dynamics. The European Physical Journal $B, 85(12): 1-8,2012$.

[37] P.L. Garrido, J.L. Lebowitz, C. Maes, and H. Spohn. Long-range correlations for conservative dynamics. Physical Review A, 42(4):1954, 1990.

[38] C. Maes and F. Redig. Long-range spatial correlations for anisotropic zero-range processes. Journal of Physics A: Mathematical and General, 24(18):4359, 1991.

[39] Z. Cheng, P.L. Garrido, J.L. Lebowitz, and J.L. Vallés. Long-range correlations in stationary nonequilibrium systems with conservative anisotropic dynamics. EPL (Europhysics Letters), 14(6):507, 1991.

[40] K.E. Bassler and Z. Rácz. Bicritical point and crossover in a two-temperature, diffusive kinetic Ising model. Physical Review Letters, 73(10):1320, 1994. 
[41] E.L. Præstgaard, B. Schmittmann, and R.K.P. Zia. A lattice gas coupled to two thermal reservoirs: Monte carlo and field theoretic studies. The European Physical Journal BCondensed Matter and Complex Systems, 18(4):675-695, 2000.

[42] A. De Masi, P.A. Ferrari, and J.L. Lebowitz. Rigorous derivation of reaction-diffusion equations with fluctuations. Physical Review Letters, 55(19):1947, 1985.

[43] M. Droz, Z. Rácz, and P. Tartaglia. One-dimensional kinetic Ising model with competing spin-flip and spin-exchange dynamics: Ordering in the case of long-range exchanges. Physical Review A, 41(12):6621, 1990.

[44] M. Droz, Z. Rácz, and P. Tartaglia. Crossover from Ising to mean-field critical behavior in a kinetic Ising model with competing flip and exchange dynamics. Physica A: Statistical Mechanics and its Applications, 177(1):401-406, 1991.

[45] H. Xu, B. Bergersen, and Z. Rácz. Long-range interactions generated by random Lévy flights: Spin-flip and spin-exchange kinetic Ising model in two dimensions. Physical Review E, 47(3):1520, 1993.

[46] M.O. Lavrentovich and R.K.P. Zia. Energy flux near the junction of two Ising chains at different temperatures. EPL (Europhysics Letters), 91(5):50003, 2010.

[47] M.O. Lavrentovich. Steady-state properties of coupled hot and cold Ising chains. Journal of Physics A: Mathematical and Theoretical, 45(8):085002, 2012.

[48] M. Pleimling, B. Schmittmann, and R.K.P. Zia. Convection cells induced by spontaneous symmetry breaking. EPL (Europhysics Letters), 89(5):50001, 2010.

[49] L. Li and M. Pleimling. Formation of nonequilibrium modulated phases under local energy input. EPL (Europhysics Letters), 98(3):30004, 2012.

[50] A.N. Kolmogorov. Math. Ann., 112:155, 1936.

[51] S.J. Cornell, K. Kaski, and R.B. Stinchcombe. Domain scaling and glassy dynamics in a one-dimensional kawasaki Ising model. Physical Review B, 44(22):12263, 1991.

[52] I.J. Helbing, D.and Farkas and T. Vicsek. Freezing by heating in a driven mesoscopic system. Physical Review Letters, 84(6):1240, 2000.

[53] R.K.P. Zia, E.L. Præstgaard, and O.G. Mouritsen. Getting more from pushing less: Negative specific heat and conductivity in nonequilibrium steady states. American Journal of Physics, 70(4):384-392, 2002.

[54] K. Binder. Finite size scaling analysis of Ising model block distribution functions. Zeitschrift für Physik B Condensed Matter, 43(2):119-140, 1981. 
[55] T. Platini. Measure of the violation of the detailed balance criterion: A possible definition of a distance from equilibrium. Physical Review E, 83(1):011119, 2011.

[56] E.T. Jaynes. Information theory and statistical mechanics. Physical Review, 106(4):620, 1957.

[57] L.D. Landau. On the theory of phase transitions. i. Zh. Eksp. Teor. Fiz., 11:19, 1937. 


\section{Appendix A}

\section{Equilibrium Ising model}

This appendix is a reproduction of the appendix found in [1]. Reprinted with permission from N.Borchers, M. Pleimling, and R.K.P. Zia, Physical Review E, 90(6):062113, 2014. Copyright 2014 by the American Physical Society.

The one-dimensional Ising model (on a ring or with open boundaries), solved in 1925 [11], appears in most textbooks on statistical mechanics. We summarize some of its properties here for the convenience of the reader. In particular, most of the texts deal with the simplest case (thermodynamic limit and no constraint on the total magnetization). Since we are not aware of any literature that displays the explicit form of say, the correlation function for a finite system with fixed $M$, we present some of its behavior here.

The partition function of the system of interest here is

$$
Z(T ; M, L)=\sum_{\left\{\sigma_{i}\right\}} \delta\left(M-\sum_{i=1}^{L} \sigma_{i}\right) \exp \left\{K \sum_{i=1}^{L} \sigma_{i} \sigma_{i+1}\right\}
$$

where $K \equiv J / k_{B} T$. The constraint prevents a simple evaluation of the configuration sum, but its generating function

$$
\Omega(M) \equiv \sum_{M} z^{M} Z(T ; M, L)
$$

can be found easily with the transfer matrix:

$$
\mathbb{T}(z, K) \equiv\left(\begin{array}{cc}
z e^{K} & z e^{-K} \\
e^{-K} / z & e^{K} / z
\end{array}\right)
$$

so that

$$
\Omega=\operatorname{Tr} \mathbb{T}^{L}
$$

Then, $Z$ can be found through the integral

$$
\frac{1}{2 \pi i} \oint \frac{d z}{z^{M+1}}\left(\lambda_{+}^{L}+\lambda_{-}^{L}\right)
$$


where

$$
\lambda_{ \pm}=e^{K}\left[\cosh H \pm \sqrt{\sinh ^{2} H+e^{-4 K}}\right]
$$

are the eigenvalues of $\mathbb{T}$, with $H \equiv \ln z$. Of course, saddle point methods can be exploited to give us $Z$ as an asymptotic expansion in large $L$.

For the two point correlation function, $G(r) \equiv\left\langle\sigma_{i} \sigma_{i+r}\right\rangle$, we have the exact expression

$$
G(r)=Z^{-1} \sum_{\left\{\sigma_{i}\right\}} \sigma_{L} \sigma_{r} \delta\left(M-\sum_{i=1}^{L} \sigma_{i}\right) \exp \left\{K \sum_{i=1}^{L} \sigma_{i} \sigma_{i+1}\right\}
$$

which can be found through

$$
\operatorname{Tr}\left\{\left(\begin{array}{cc}
1 & 0 \\
0 & -1
\end{array}\right) \mathbb{T}^{r}\left(\begin{array}{cc}
1 & 0 \\
0 & -1
\end{array}\right) \mathbb{T}^{L-r}\right\}
$$

and another, somewhat more involved, integral. Instead of pursuing these exact expressions, it is reasonable to appeal to an approximation, which should be quite good here. We start with the exact expression

$$
G_{0}(r)=\left(\omega^{r}+\omega^{L-r}\right) /\left(1+\omega^{L}\right)
$$

where $\omega \equiv \tanh K$, for a finite ring without constraints on $M$. Assuming a linear relationship between $G_{0}$ and our $G$, we fix the constants by imposing $G(0)=1$ and the constraint

$$
\sum_{r=0}^{L-1} G(r)=M^{2} / L
$$

(a consequence of $M=\sum_{i=1}^{L} \sigma_{i}$ ). The result is

$$
G(r) \cong \frac{G_{0}(r)-B}{1-B}
$$

with

$$
B=\frac{1}{L^{2}-M^{2}}\left[L \frac{\left(1-\omega^{L}\right)(1+\omega)}{\left(1+\omega^{L}\right)(1-\omega)}-M^{2}\right]
$$

Thus,

$$
G(r) \cong \frac{\left(\omega^{r}+\omega^{L-r}\right)-\left(1+\omega^{L}\right) B}{\left(1+\omega^{L}\right)(1-B)}
$$

which is the form (3.3). If we impose $M=0$ and consider $r$ 's such that $\omega^{L-r} \ll \omega^{r}$, then

$$
B \rightarrow e^{2 K} / L
$$

and

$$
G(r) \cong \frac{\omega^{r}-e^{2 K} / L}{1-e^{2 K} / L}
$$


Turning to the distribution $\mathcal{P}_{w}(m)$, it is given by

$$
\mathcal{P}_{w}(m, M)=Z^{-1} \sum_{\left\{\sigma_{i}\right\}} \delta\left(m-\sum_{i=1}^{w} \sigma_{i} / w\right) \delta\left(M-\sum_{i=1}^{L} \sigma_{i}\right) \exp \left\{K \sum_{i=1}^{L} \sigma_{i} \sigma_{i+1}\right\}
$$

Its generating function

$$
\Omega_{w}(\zeta ; z) \equiv \sum_{m, M} \zeta^{m w} z^{M} \mathcal{P}_{w}(m, M)
$$

can be computed as above:

$$
\operatorname{Tr}\left[\mathbb{T}(\zeta z, K)^{w} \mathbb{T}(z, K)^{L-w}\right]
$$

while inverting it to $\mathcal{P}_{w}$ will require two contour integrals. Though feasible, we will not pursue these results here. Simulations provide a more direct route to $\mathcal{P}_{w}$, and we are not interested in its analytic properties. 


\section{Appendix B}

\section{Some remarks on a possible phase transition}

For continuous (i.e. second-order) phase transitions there are a host of techniques available by which the details of the transition can be explored. Many of these rely on Landau's theory of these phase transitions [57], which is based on an expansion of the free energy of the system in terms of an order parameter which quantifies the degree of order in the system. Such a quantity will be zero above the critical temperature $T_{c}$, but will increase continuously once below. The exact quantity to be used as the order parameter will depend intimately on the nature of the system being considered. For the standard Ising model, which was one of the first systems that Landau considered, a natural choice of order parameter is the average magnetization per spin, $m_{N}=\langle M\rangle / N$. Ideally, above $T_{c}$ this quantity is zero, while below it can be shown that $m_{N} \propto \sqrt{1-T / T_{c}}$. This behavior is shown in Fig. B.1.

For an Ising system of finite system size, such as any system which is analyzed computational via a Monte Carlo simulation, this result is only approximate. In the first place, $m_{N}$ does not serve as an appropriate order parameter, since the spin symmetry assures that an ensemble average of this quantity will always be zero. To rectify this, the absolute value of this quantity is typically cosidered instead. Even with a suitably chosen quantity, in finite systems the order parameter goes to zero in a more gradual manner above $T_{c}$, and doesn't quite follow the $\sqrt{1-T / T_{c}}$ curve below.

Though the $2 T$ system is not amenable to Landau theory (there is no free energy to minimize to indicate the correct steady state, for instance), it is still possible to choose a quantity which behaves in a similar manner to an order parameter. Since the ordered state of the system is indicated by a non-zero value of the normalized window magnetization $m$, this is a natural choice. As with any Ising system, an absolute value must be applied to deal with the spin symmetry. In Fig. B.2, the average of the absolute value of $m$ is tracked as $T$ is varied for a number of system sizes. For the smaller system sizes, the behavior is at least qualitatively similar to that seen in Fig. B.1, and the curves even display the anticipated effects of finite 


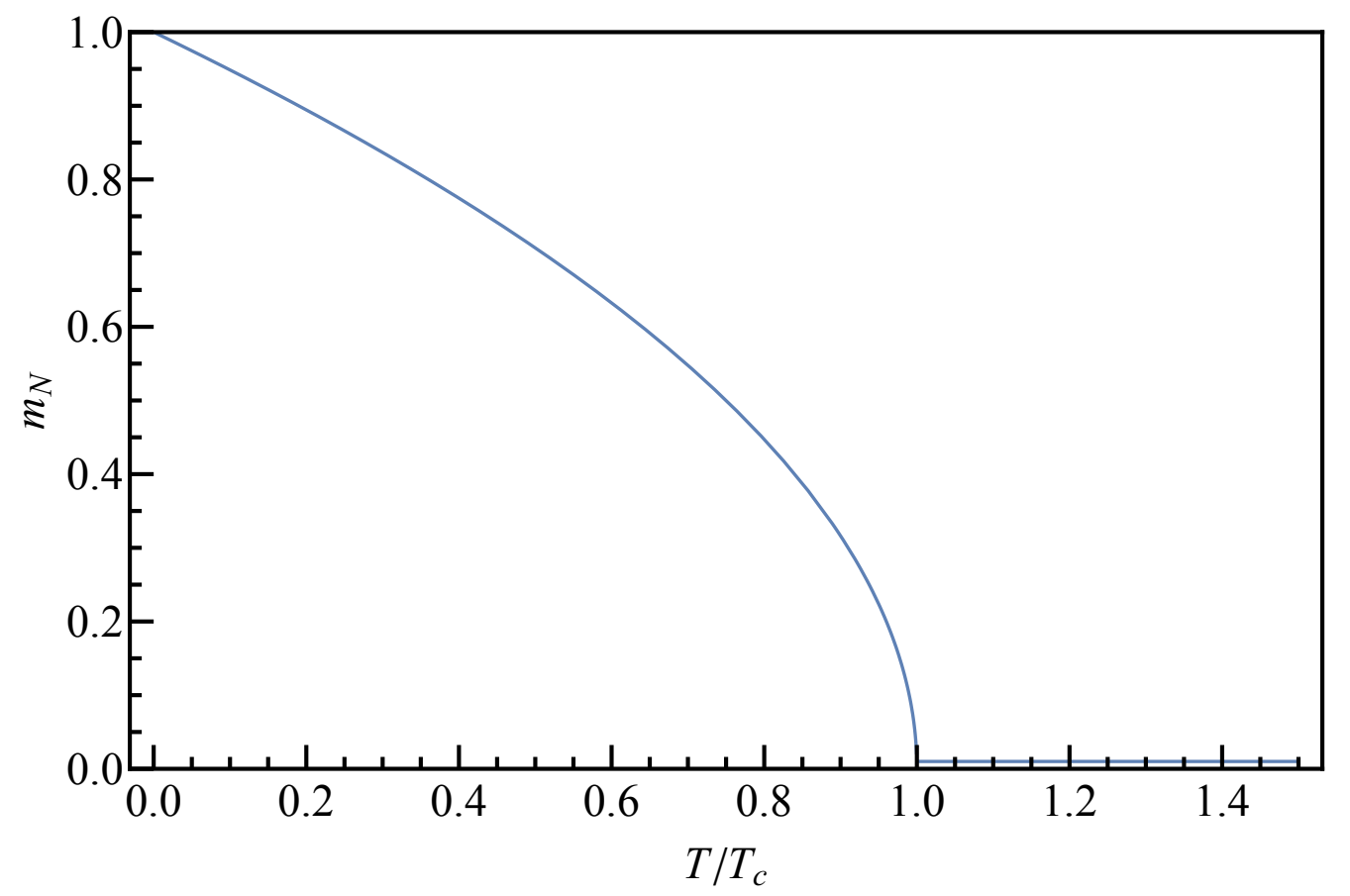

Figure B.1: The ideal behavior of the order parameter for a continuous phase transition as temperature is varied.

size effects at temperatures above the apparent value of $T_{c}$. For the largest system size $L=512$, however, there is an extreme deviation at $T \approx 1.8$. Not only is the value of $\langle|m|\rangle$ grossly different than the other curves, but the error bars increase dramatically.

These results, when first produced, were (unsurprisingly) considered an indication of poor data quality. Surely such extremely aberrant results must be the consequence of some flaw in the generation of the data! In light of the discovery of the bistable steady state, this result makes perfect sense. If the distribution of $|m|$ has two peaks rather than the anticipated one, error bars would increase dramatically when considering the average of this distribution. Furthermore, the values of $\langle|m|\rangle$ in this vicinity would match very well with two states being ordered $(|m|=1)$ and disordered $(m=0)$.

Before the role of the bistability was recognized and the crossover was still speculated to be second order, the Binder cumulant, $U$, was employed to try and find a precise measurement of $T_{c}$ [54]. This quantity,

$$
U=1-\frac{\left\langle m^{4}\right\rangle}{3\left\langle m^{2}\right\rangle^{2}}
$$




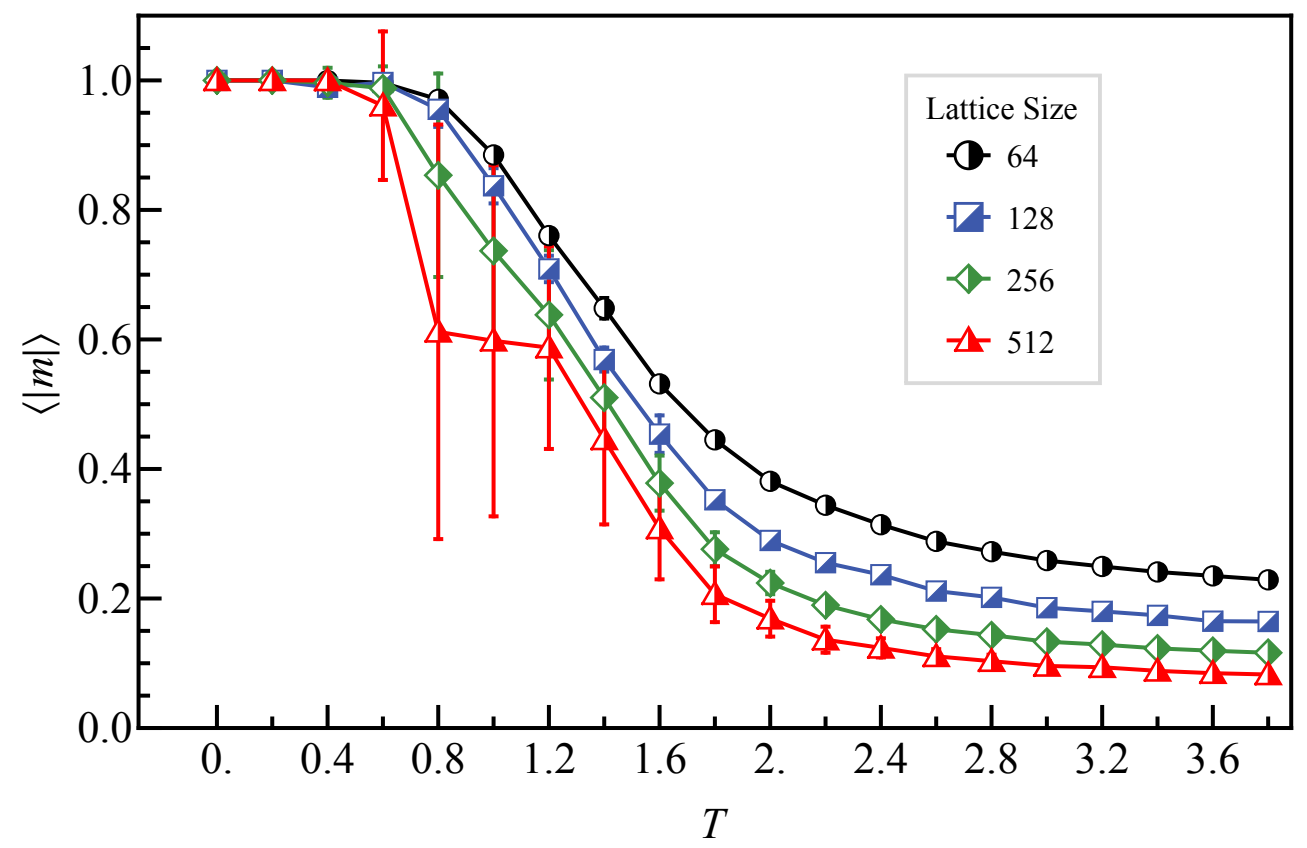

Figure B.2: The response of the $2 T$ order parameter $|m|$ as $T$ is varied near the crossover temperature. Several system sizes are considered.

is essentially a rescaled kurtosis, or fourth moment, of the magnetization distribution. Since this distribution should tend to a universal form at the critical point of a continuous transition, the value of $U$ at $T_{c}$ should be the same independent of the system size. One may then use this fact to pin down the critical temperature by looking for the point at which curves originating from different system sizes converge. This is, of course, assuming that the transition is actually second order. For the $2 T$ system, which has displayed qualities indicating it is anything but a standard continuous transition, Fig. B.3 shows this quantity for a number of system sizes.

While there are no consistent crossings to indicate where $T_{c}$ is, the curves for $L<256$ at least behave in the expected manner. For larger values of $L$, however, the curves again show highly divergent behavior for $0.7<T<1.1$. With the knowledge that the system enters the bistable regime within this parameter range, these results can be readily understood.

While the techniques discussed in this appendix present powerful means for analyzing continuous transitions, it is clear that the unique nature of the steady state in the $2 T$ model will require different methodologies for developing a more complete understanding of the crossover. 


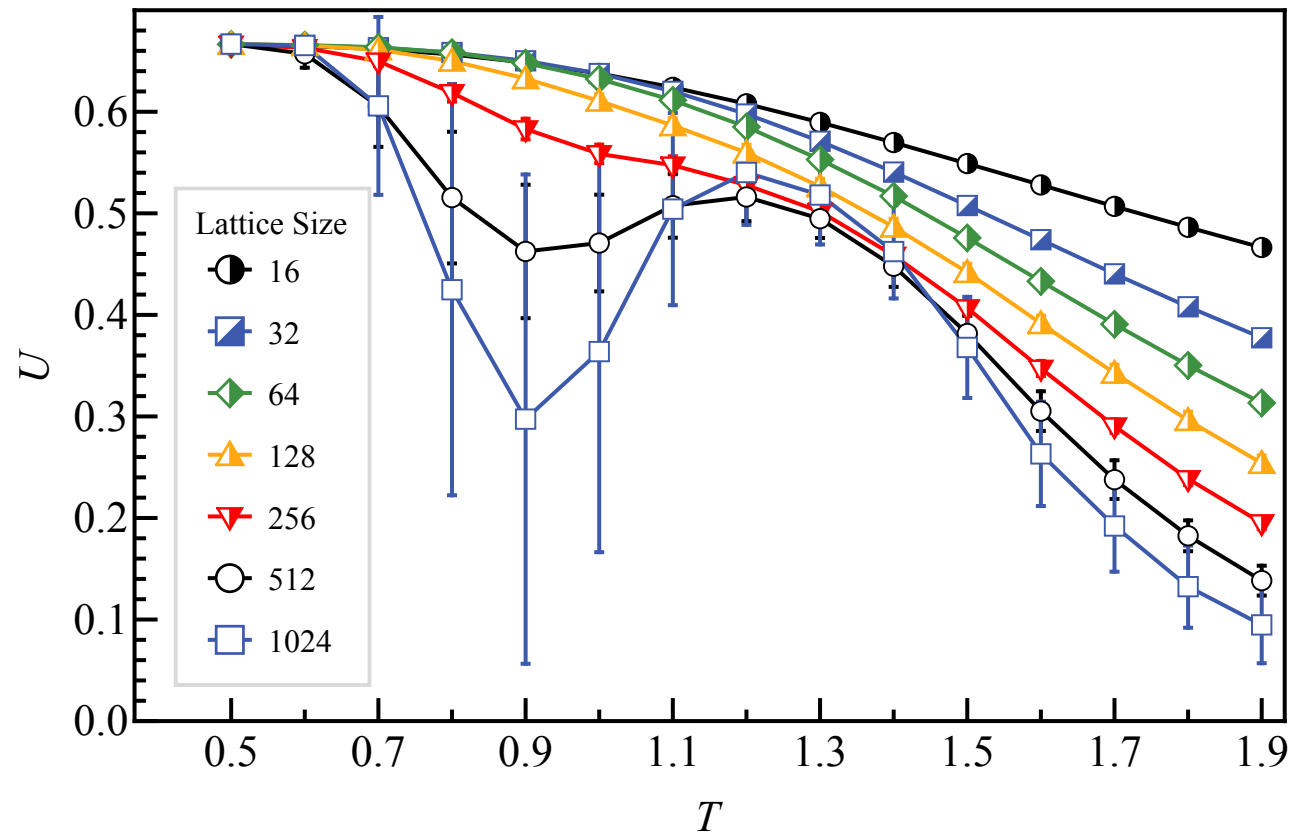

Figure B.3: The Binder cumulant for the $2 T$ system as a function of $T$. Several system sizes are considered. 